\title{
Identification and characterization of point defects in aluminum nitride and zinc oxide crystals
}

\author{
Sean M. Evans \\ West Virginia University
}

Follow this and additional works at: https://researchrepository.wvu.edu/etd

\section{Recommended Citation}

Evans, Sean M., "Identification and characterization of point defects in aluminum nitride and zinc oxide crystals" (2008). Graduate Theses, Dissertations, and Problem Reports. 2705.

https://researchrepository.wvu.edu/etd/2705

This Dissertation is protected by copyright and/or related rights. It has been brought to you by the The Research Repository @ WVU with permission from the rights-holder(s). You are free to use this Dissertation in any way that is permitted by the copyright and related rights legislation that applies to your use. For other uses you must obtain permission from the rights-holder(s) directly, unless additional rights are indicated by a Creative Commons license in the record and/ or on the work itself. This Dissertation has been accepted for inclusion in WVU Graduate Theses, Dissertations, and Problem Reports collection by an authorized administrator of The Research Repository @ WVU.

For more information, please contact researchrepository@mail.wvu.edu. 


\title{
Identification and Characterization of Point Defects in Aluminum Nitride and Zinc Oxide Crystals
}

\author{
Sean M. Evans \\ Dissertation submitted to the \\ Eberly College of Arts and Sciences \\ at West Virginia University \\ in partial fulfillment of the requirements \\ for the degree of \\ Doctor of Philosophy \\ in \\ Physics \\ Larry E. Halliburton, Ph.D., Chair \\ Nancy C. Giles, Ph.D. \\ Leonardo Golubovic, Ph.D. \\ David Lederman, Ph.D. \\ Sendhil Velan, Ph.D. \\ Department of Physics \\ Morgantown, West Virginia \\ 2008
}

Keywords: EPR, ENDOR, points defects, ZnO, AlN

Copyright 2008 Sean M. Evans 


\begin{abstract}
Identification and Characterization of Point Defects in Aluminum Nitride and Zinc Oxide Crystals
\end{abstract}

Sean M. Evans

Electron paramagnetic resonance (EPR) and electron-nuclear double resonance (ENDOR) studies have been performed on single crystals of aluminum nitride (AIN) and zinc oxide $(\mathrm{ZnO})$, two wide-band-gap semiconductors having the wurtzite crystal structure. These studies were used to characterize point defects in each material. In the first study in AlN, new EPR and ENDOR spectra were acquired from a deep donor. Although observed in as-grown crystals, exposure to x rays significantly increased the concentration of this center. ENDOR identified a strong hyperfine interaction with one aluminum neighbor along the $c$ axis and weaker equivalent hyperfine interactions with three additional aluminum neighbors in the basal plane. These aluminum interactions indicate that the responsible center was located at a nitrogen site. The observed paramagnetic defect is either an oxygen substituting for nitrogen or a nitrogen vacancy. An analysis of the hyperfine data suggests that substitutional oxygen is the most likely candidate.

The second point defect studied in AlN was silicon substituting for aluminum. Silicon is a shallow donor in AlN, and its neutral charge state is paramagnetic. Two samples containing silicon were studied. Only one of the samples was intentionally doped with silicon. The silicon-related EPR signals from these two samples had different behaviors. The signal from the doped sample had behavior similar to that described in previous studies where the silicon was explained as a DX center. The undoped sample had behavior that was inconsistent with a DX center.

In $\mathrm{ZnO}, \mathrm{EPR}$ was used to monitor oxygen vacancies and zinc vacancies in a $\mathrm{ZnO}$ crystal irradiated near room temperature with $1.5 \mathrm{MeV}$ electrons. Out-of-phase detection at $30 \mathrm{~K}$ greatly enhanced the EPR signals from these vacancies. Following the electron irradiation, but before illumination, $\mathrm{Fe}^{3+}$ ions and nonaxial singly ionized zinc vacancies were observed. Illumination with $325 \mathrm{~nm}$ laser light at low temperature eliminated the $\mathrm{Fe}^{3+}$ signal while producing spectra from singly ionized oxygen vacancies, neutral zinc vacancies, and axial singly ionized zinc vacancies. This light also produced EPR spectra from zinc vacancies having an $\mathrm{OH}^{-}$ion at an adjacent oxygen site. The low temperature response of the irradiated crystal to illumination wavelengths between 350 and $750 \mathrm{~nm}$ is described. Wavelengths shorter than $600 \mathrm{~nm}$ converted $\mathrm{Fe}^{3+}$ ions to $\mathrm{Fe}^{2+}$ ions and converted neutral oxygen vacancies to singly ionized oxygen vacancies. Neutral zinc vacancies were formed by wavelengths shorter than $500 \mathrm{~nm}$ as electrons were removed from isolated singly ionized zinc vacancies. Warming above $120 \mathrm{~K}$ in the dark reversed the effect of the illuminations. These wavelength-dependence results suggest that the ground state of the neutral oxygen vacancy is deep, approximately $1.3 \mathrm{eV}$ above the 
valence band, and that the ground state of the singly ionized zinc vacancy is also deep, about $0.9 \mathrm{eV}$ above the valence band.

The hyperfine associated with the isolated nitrogen acceptor in $\mathrm{ZnO}$ was studied with EPR. The sample used in this study was grown by the seeded chemical vapor transport method, with $\mathrm{N}_{2}$ added to the gas stream to serve as the doping source. This study further characterized the hyperfine interactions with the nitrogen nucleus $(I=1)$ and the nearest-neighbor zinc nuclei $(I=5 / 2)$. Angular dependence data were obtained from EPR and were analyzed by complete diagonalizations of the full spin Hamiltonian. Nuclear electric quadrupole effects were included in the nitrogen hyperfine analysis, thus yielding a value for the nuclear quadrupole and more accurate $g$ values and nitrogen hyperfine parameters. 


\section{Acknowledgments}

I would first like to acknowledge and thank my advisor, Dr. Larry Halliburton, for allowing me to work in his lab and giving me the opportunity to work on a variety of research projects. His guidance not only taught me many problem solving skills, but has also been a tremendous help in developing a future direction for my career. His patience in answering my many questions dealing with research and non-research related questions over the years has been greatly appreciated.

I would also like to thank my committee members Dr. Nancy Giles, Dr. Leonardo Golubovic, Dr. David Lederman, and Dr. Sendhil Velan for their willingness to serve on my committee and for their guidance. I would also like to thank Dr. Giles for use of her EPR and FTIR instruments, and for the many interactions that gave me a chance to learn more about the optical properties of materials. On many occasions, she patiently answered my many questions on various topics.

I would also like to acknowledge others that contributed to my research. I would like to express my gratitude to Drs. Glen Slack, Leo Schowalter, and Sandra Schujman at Crystal IS for providing aluminum nitride samples used in this work. Dr. Larry Kappers at the University of Connecticut was kind enough to take time to use his Van de Graff accelerator to electron irradiate a sample of zinc oxide.

Most of all, I would like to acknowledge the support I received during my lengthy education from my friends and family. My parents, my brothers, and my wife were all extremely supportive and unconditionally encouraging during this time.

My research was supported by the National Science Foundation under Grant No. DMR-0508140 and by the West Virginia STEM Fellowship Program. A special thanks goes to Dr. Peter Gannett for his efforts in establishing and managing the STEM fellowship program at WVU. 


\section{Table of Contents}

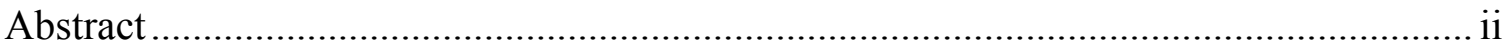

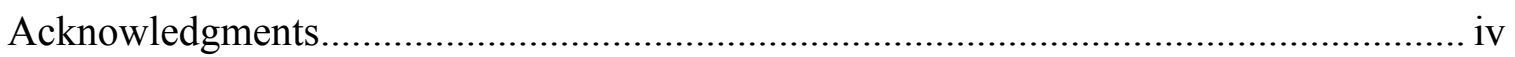

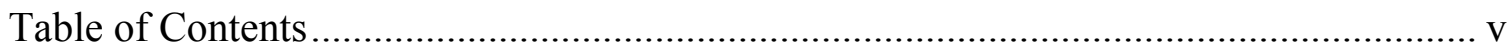

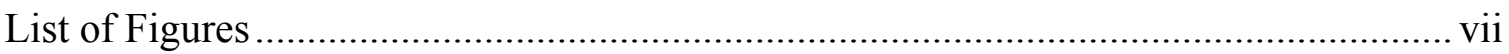

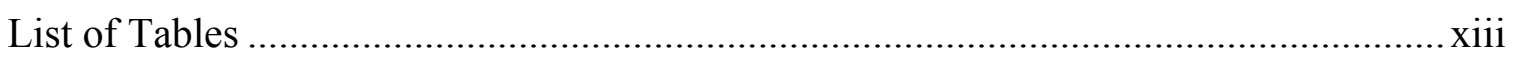

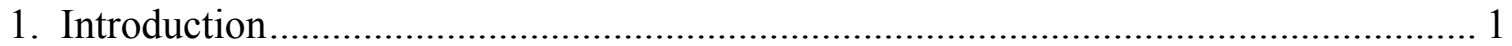

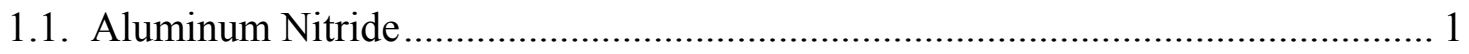

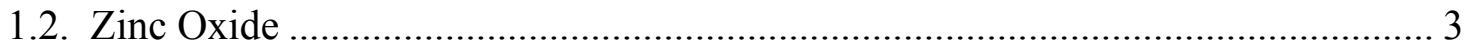

2. EPR and ENDOR: Principles and Instrumentation .................................................... 7

2.1. Electron Paramagnetic Resonance ....................................................................... 7

2.1.1. Basic Principles of EPR ………………………...................................... 8

2.1.2. Hyperfine Interactions with Nuclei............................................................... 14

2.1.3. EPR Transitions and Probabilities ................................................................ 18

2.2. Electron-Nuclear Double Resonance.................................................................. 20

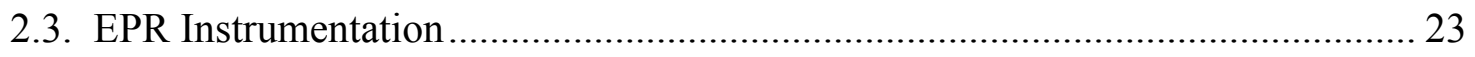

2.4. ENDOR Instrumentation .............................................................................. 29

3. Oxygen Donors in Aluminum Nitride …………………....................................... 30

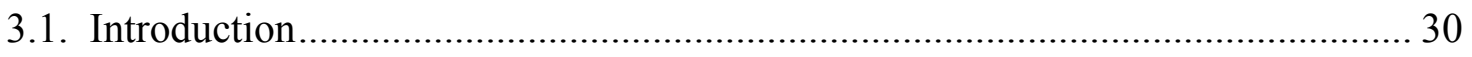

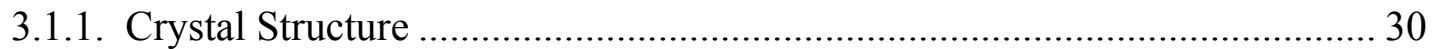

3.1.2. Crystallographically Equivalent Defect Orientations .................................... 32

3.1.3. Previous Defect Studies in Aluminum Nitride ............................................. 35

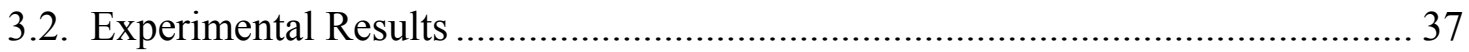

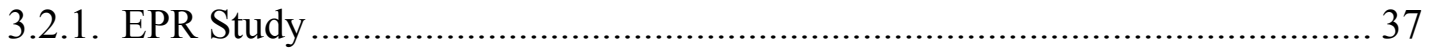

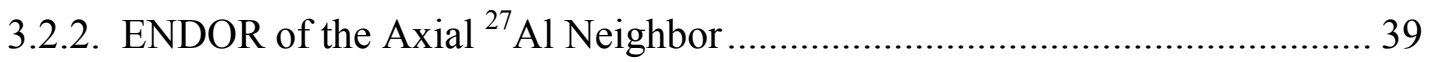

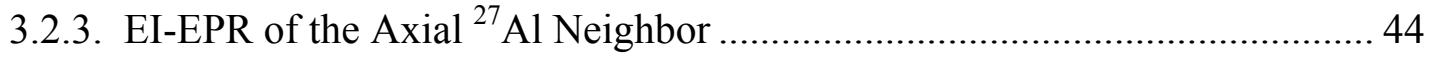

3.2.4. Additional ENDOR at Low Frequency........................................................ 46

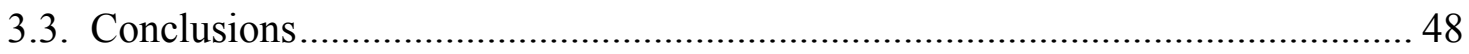




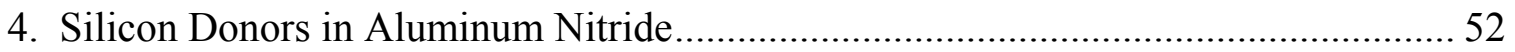

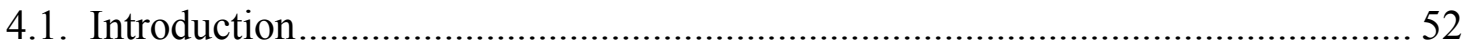

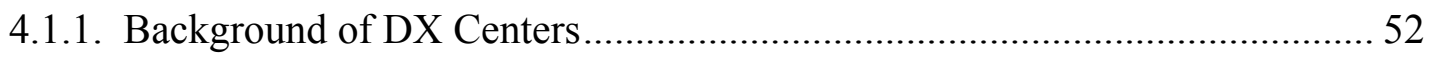

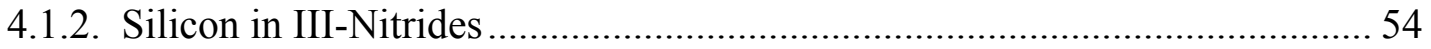

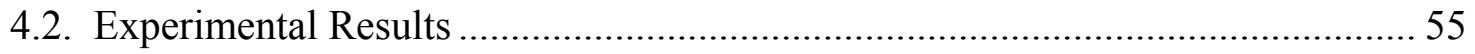

4.2.1. Optical Absorption and EPR Signal .......................................................... 56

4.2.2. Photoexcitation Wavelength Dependence ……………….............................. 59

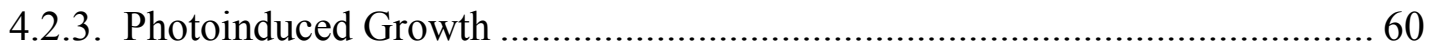

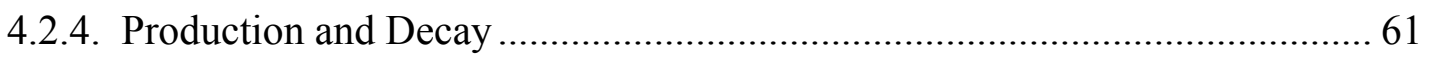

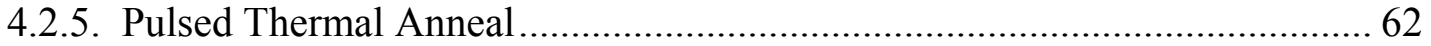

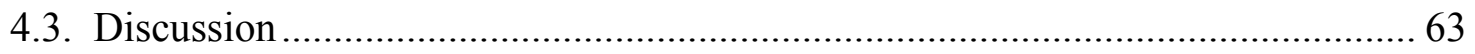

5. Oxygen Vacancies and Zinc Vacancies in Zinc Oxide............................................... 69

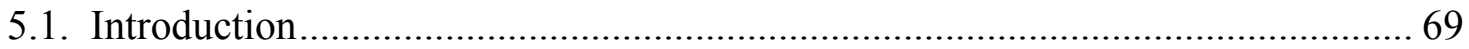

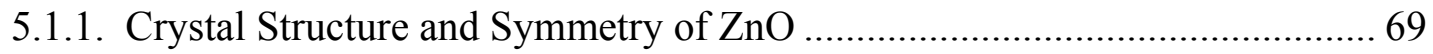

5.1.2. Growth of Bulk ZnO Crystals..................................................................... 70

5.1.3. Previous Studies of Vacancies in $\mathrm{ZnO}$........................................................ 71

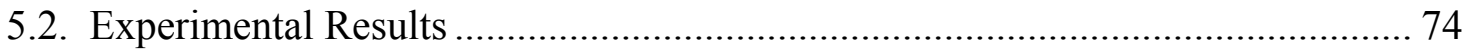

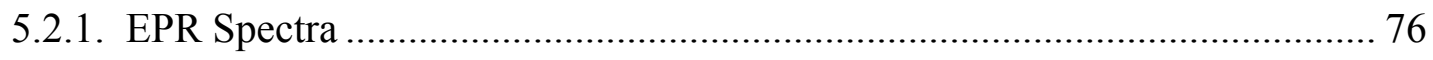

5.2.2. Wavelength Dependence of Photoinduced Effects......................................... 83

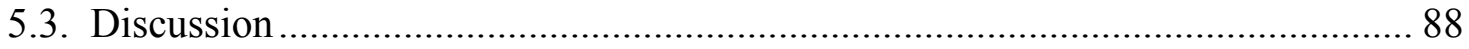

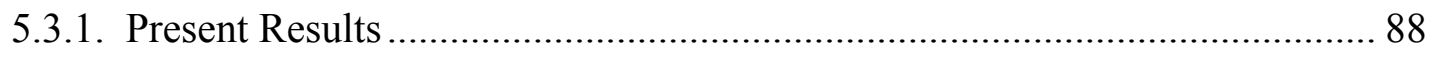

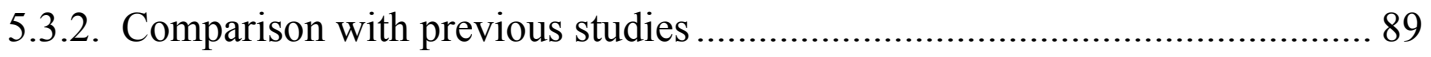

6. Nitrogen Acceptors in Zinc Oxide ………………….................................................. 95

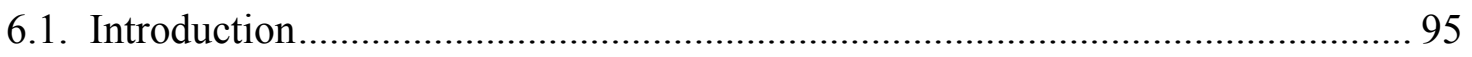

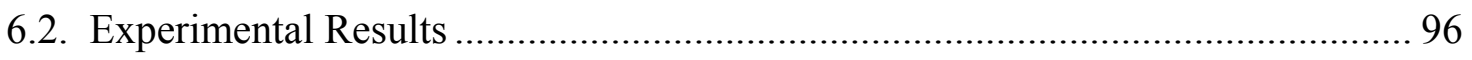

6.2.1. Nitrogen Hyperfine ………………………………..................................... 96

6.2.2. Zinc Hyperfine ....................................................................................... 98

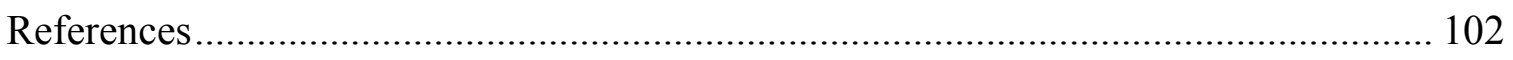




\section{List of Figures}

Figure 2.1. Energy level splitting for an $\mathrm{S}=1 / 2$ system in an increasing magnetic field. The vertical arrow represents the resonance position.

Figure 2.2. Energy level diagram of an $\mathrm{S}=1$ spin system. Solid vertical lines represent the allowed transitions $\left(\Delta \mathrm{m}_{\mathrm{S}}= \pm 1\right)$.

Figure 2.3. Energy level diagram of an $S=1 / 2, I=1 / 2$ system. Solid lines represent the energy levels that are split by the hyperfine interaction. Dashed lines represent the energy levels in the absence of a hyperfine interaction. The solid and dashed vertical arrows represent EPR transitions with and without hyperfine interactions, respectively.

Figure 2.4. Energy level diagram for $\mathrm{A} / 2>v_{\mathrm{N}}$. Included are the electron-Zeeman, hyperfine, and nuclear-Zeeman effects. The double arrows represent EPR transitions, while the heavy solid arrows represent ENDOR transitions.

Figure 2.5. Energy level diagram for $v_{\mathrm{N}}>\mathrm{A} / 2$. Included are the electron-Zeeman, hyperfine, and nuclear-Zeeman effects. The double arrows represent EPR transitions, while the heavy solid arrows represent ENDOR transitions.

Figure 2.6. Diagrams of the population of spin states during different stages of EPR and ENDOR. (a) The states are initially in thermal equilibrium. (b) The population of states under a saturated EPR transition between states A and D. (c) The states after an induced nuclear transition between $C$ and D. (d) The population of states after the EPR signal reaches a new equilibrium. (after Spaeth ${ }^{47}$ ).....

Figure 2.7. Diagram of a typical EPR system. Components 1, 2, 3, 6, and 7 all reside in the microwave bridge 
Figure 2.8. (a) Schematic of a $\mathrm{TE}_{102}$ rectangular cavity. (b) Electric field lines inside the cavity. (c) Magnetic field lines inside the cavity. (after Wertz and Bolton ${ }^{49}$ ).......... 26

Figure 2.9. (a) Schematic of a $\mathrm{TE}_{011}$ cylindrical cavity. (b) Electric field lines inside the cavity. (c) Magnetic field lines inside the cavity.

Figure 2.10. (a) Illustration of the effect of the field modulation on the amplitude of the microwave signal. (b) First-derivative output signal of the lock-in amplifier. $\left(\right.$ after Setzler ${ }^{48}$. 28

Figure 3.1. Unit cell of aluminum nitride. (a) Three-dimensional view. (b) Projection on the basal plane. 30

Figure 3.2. Projection of the AlN crystal structure on the basal plane. Solid arrows indicate the crystal high-symmetry directions $\left(\mathrm{a}_{1}, \mathrm{a}_{2}, \mathrm{a}_{3}\right)$, while the dashed lines $(\mathrm{X}, \mathrm{Y})$ indicate the orthogonal crystal coordinate system.

Figure 3.3. EPR from a donor in a single crystal of AlN. The sample was irradiated with $\mathrm{x}$ rays at room temperature to increase the intensity of the EPR signal. These data were taken at $40 \mathrm{~K}$ with the magnetic field (a) parallel to the $c$ axis and (b) perpendicular to the $c$ axis. The microwave frequency was $9.480 \mathrm{GHz}$.

Figure 3.4. Integration of the spectra in Fig. 3.3. This shows the top-hat shape of the absorption curve for the magnetic field (a) parallel to the $c$ axis and (b) perpendicular to the $c$ axis.

Figure 3.5. An ENDOR spectrum from AlN showing the primary aluminum hyperfine interaction. These data were taken at $22 \mathrm{~K}$ with the magnetic field (3382 $\mathrm{G})$ perpendicular to the $c$ axis. The five lines in each set result from the nuclear electric quadrupole interaction with the $\mathrm{I}=5 / 2{ }^{27} \mathrm{Al}$ nucleus. 
Figure 3.6. Energy level diagram of a spin system with $S=1 / 2$ and $I=5 / 2$. The diagram shows the splitting caused by each term in the spin Hamiltonian. Each energy level is labeled by the appropriate $m_{I}$ value. EPR and ENDOR transitions are indicated by the double and single arrows, respectively. Here, the parameters A, Q, and $v_{\mathrm{N}}$ are taken to be positive and $\mathrm{A} / 2$ is greater than $v_{\mathrm{N}}$

Figure 3.7. Angular dependence of the primary aluminum ENDOR spectrum. The magnetic field was rotated from the $c$ axis $\left(0^{\circ}\right)$ to the basal plane $\left(90^{\circ}\right)$. The solid lines are calculated using the best fit parameters and the discrete points represent experimental data

Figure 3.8. EI-EPR data (solid curves) taken using three ENDOR signals of the axial ${ }^{27} \mathrm{Al}$ neighbor. Spectra were taken at $20 \mathrm{~K}$ with the magnetic field aligned perpendicular to the $c$ axis. Each solid curve represents two unresolved EPR transitions (dashed curves) 46

Figure 3.9. ENDOR spectrum of the basal plane ${ }^{27} \mathrm{Al}$ neighbors associated with the donor defect EPR signal. These data were taken at $38 \mathrm{~K}$ with the magnetic field (3381 G) parallel to the $c$ axis.

Figure 3.10. Diagram of the location of the defect. There is a primary hyperfine interaction with the axial aluminum atom, and three equivalent interactions with the basal plane aluminum atoms.

Figure 4.1. Comparison of the optical absorption of undoped (left) and doped (right) samples.

Figure 4.2. EPR spectra taken from the undoped sample (a) at room temperature with no light, and (b) at $20 \mathrm{~K}$ during illumination with $442 \mathrm{~nm}$ light. 
Figure 4.3. EPR of (a) undoped and (b) doped samples under illumination at $20 \mathrm{~K}$ with $442 \mathrm{~nm}$ light

Figure 4.4. Wavelength dependence of the production of the photoinduced silicon EPR signal from the undoped (left) and doped (right) samples.

Figure 4.5. Growth curves for the silicon EPR signal in the undoped (left) and doped (right) samples. Data were taken at $35 \mathrm{~K}$ with $400 \mathrm{~nm}$ excitation light at $0.74 \mathrm{~mW}$ power.

Figure 4.6. Growth and decay curves for the undoped (left) and doped (right) samples. Samples were illuminated at $35 \mathrm{~K}$ with $442 \mathrm{~nm}$ laser light and the light was shuttered once the signal reached saturation.

Figure 4.7. Pulsed thermal anneal results for the silicon EPR signal in the undoped (left) and doped (right) AIN samples.

Figure 5.1. (a) Model of the non-axial zinc vacancy showing the trapped hole on a neighboring basal plane oxygen atom. Note the three possible sites for the trapped hole. (b) Model of the axial zinc vacancy with the hole trapped on the axial oxygen atom

Figure 5.2. EPR spectra taken at $30 \mathrm{~K}$ from a $\mathrm{ZnO}$ crystal irradiated with $1.5 \mathrm{MeV}$ electrons. No illumination was used. The magnetic field was (a) parallel to the $c$ axis and (b) perpendicular to the $c$ axis (at an arbitrary angle in the basal plane). 76

Figure 5.3. EPR spectra taken at $30 \mathrm{~K}$ from a $\mathrm{ZnO}$ crystal irradiated with $1.5 \mathrm{MeV}$ electrons. (a) Before illumination. (b) After a brief exposure to $325 \mathrm{~nm}$ laser light. ..... 78

Figure 5.4. Expanded view of the center portion of the EPR spectrum in Fig. 5.3(b), showing four axial zinc-vacancy centers. The lower stick diagram illustrates 
forbidden transitions associated with Center D. A small signal from $\mathrm{Fe}^{3+}$ ions is also present.

Figure 5.5. EPR spectrum of the neutral zinc vacancy $\left(\mathrm{V}_{\mathrm{Zn}}^{0}\right)$ in an electron-irradiated $\mathrm{ZnO}$ crystal. These data were taken at $30 \mathrm{~K}$ after a brief exposure to $325 \mathrm{~nm}$ laser light.

Figure 5.6. Wavelength dependence of changes in charge state while illuminating at $30 \mathrm{~K}$. The monitored EPR signals are (a) $\mathrm{Fe}^{3+}$ ions, (b) singly ionized oxygen vacancies $\left(\mathrm{V}_{\mathrm{O}}^{+}\right)$, and (c) zinc vacancies with an $\mathrm{OH}^{-}$ion at an adjacent oxygen site, i.e., $\left(\mathrm{V}_{\mathrm{Zn}}^{-}-\mathrm{H}^{+}\right)^{0}$ centers.

Figure 5.7. Time dependence of changes in charge state while illuminating at $30 \mathrm{~K}$ with $325 \mathrm{~nm}$ laser light. The monitored EPR signals are (a) $\mathrm{Fe}^{3+}$ ions, (b) singly ionized oxygen vacancies $\left(\mathrm{V}_{\mathrm{O}}^{+}\right)$, and (c) zinc vacancies with an $\mathrm{OH}^{-}$ion at an adjacent oxygen site, i.e., $\left(\mathrm{V}_{\mathrm{Zn}}^{-}-\mathrm{H}^{+}\right)^{0}$ centers.

Figure 6.1. EPR spectra of the isolated neutral nitrogen acceptor taken at $5 \mathrm{~K}$ during illumination with $442 \mathrm{~nm}$ laser light. The magnetic field (a) along the $c$ axis, (b) perpendicular to the $c$ axis along the high symmetry direction labeled "hs1", and (c) perpendicular to the $c$ axis along the high symmetry direction labeled "hs2".

Figure 6.2. Angular dependence of the neutral nitrogen acceptor. Solid circles represent experimental data points. The solid lines and dashed lines represent the computer generated line positions of the "allowed transitions" (solid lines) and "forbidden transitions" (dashed lines) using the "best fit" parameters.

Figure 6.3. EPR spectra of the zinc hyperfine. The spectra were taken with the magnetic field (a) parallel to the $c$ axis, (b) perpendicular to the $c$ axis along "hs 1 ", and (c) perpendicular to the $c$ axis along "hs2". 
Figure 6.4. Angular dependence of (a) the axial zinc hyperfine and (b) the non axial zinc hyperfine. The data points represent experimental data, while the solid lines are computer generated curves using the "best fit" parameters. 


\section{List of Tables}

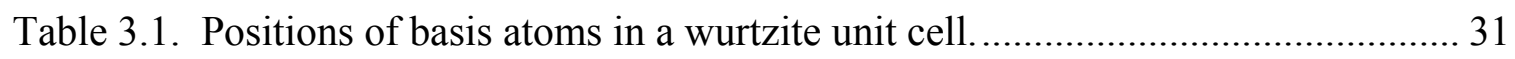

Table 3.2. Positions of basis atoms in a unit cell of AlN ........................................... 31

Table 3.3. Levels and energies for an $\mathrm{S}=1 / 2, \mathrm{I}=5 / 2$ spin system. The results in this table are based on the energy level diagram in Fig. 3.6 ............................................ 42

Table 3.4. Frequencies for ENDOR transitions associated with an $S=1 / 2, I=5 / 2$

spin system. The results in this table are based on the energies in Table 3.3 ................ 42

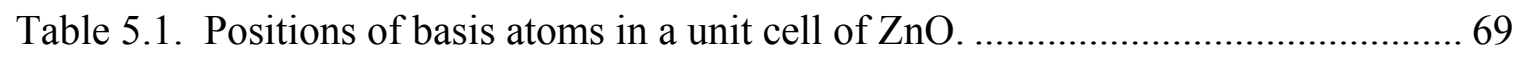




\section{CHAPTER 1 \\ Introduction}

Efforts beginning in the early 1990s and continuing today have focused attention on the development of compound semiconductors for short-wavelength light emission, detection, and sensor applications. The control of point defects (i.e., donors and acceptors) either in the form of impurities or native defects is the key to the fabrication of useful devices in this class of materials. This dissertation describes a series of fundamental investigations of donors and acceptors in two important wide-band-gap semiconductors, aluminum nitride (AlN) and zinc oxide $(\mathrm{ZnO})$.

\subsection{Aluminum Nitride}

Research is presently underway in many laboratories to develop wide-band-gap semiconductors for use in devices operating in the 210 to $350 \mathrm{~nm}$ region of the ultraviolet

spectrum. A major portion of this effort is focused on the $\mathrm{Al}_{\mathrm{x}} \mathrm{Ga}_{1-\mathrm{x}} \mathrm{N}$ system. ${ }^{1,2}$ The band gap of the material increases as the aluminum content increases, reaching $6.2 \mathrm{eV}(200$ $\mathrm{nm}$ ) when $\mathrm{x}=1$. Numerous applications of ultraviolet semiconductor devices become possible as this materials system matures. Light-emitting diodes (LEDs) operating at these short wavelengths will significantly impact the development of fluorescence-based biochemical sensors, and can also be used for purification and sterilization. Ultraviolet laser diodes, operating at these short wavelengths, offer the potential to match output wavelengths to peak absorption wavelengths of specific biochemical species, and thus greatly enhance the detection of their fluorescence signals. Solar-blind detectors are another area of application. $\mathrm{The} \mathrm{Al}_{\mathrm{x}} \mathrm{Ga}_{1-\mathrm{x}} \mathrm{N}$ films needed for these ultraviolet devices are challenging to fabricate, and problems encountered when producing devices include $\mathrm{p}$ dopants, dislocations, lifetimes, contacts, transparency, defect fluorescence, etc. Much of today's active research is focused on the donors and acceptors in aluminum nitride crystals (i.e, the short wavelength $x=1$ end of the $\mathrm{Al}_{\mathrm{x}} \mathrm{Ga}_{1-\mathrm{x}} \mathrm{N}$ system). Questions about 
the transparency of the material in the ultraviolet, the nature of the luminescence bands, and the difficulty of $\mathrm{p}$ doping must be answered before devices are routinely deployed.

In addition to devices, AIN has an equally important role to play as a substrate for growth of thin films of gallium nitride $(\mathrm{GaN})$ and its aluminum alloys. Improvement in the optical properties of AlN substrates may, in turn, lead to improvement in $\mathrm{Al}_{\mathrm{x}} \mathrm{Ga}_{1-\mathrm{x}} \mathrm{N}$ film growth and device performance. The alternatives to the use of AIN are sapphire $\left(\mathrm{Al}_{2} \mathrm{O}_{3}\right)$ and silicon carbide $(\mathrm{SiC})$, but neither is acceptable for the long-term success of nitride devices. Single crystals of AlN are vastly superior to the other substrate candidates because AlN (1) can be grown as large crystals, (2) provides an excellent lattice match to GaN, (3) has high thermal conductivity (which allows high power operation), (4) has low dislocation densities, and (5) is easily grown in semi-insulating form.

The optical and electrical properties of bulk AlN crystals are strongly affected by the presence of donors and acceptors. These defects can be shallow (e.g., silicon impurities $^{3}$ ), but they are more often deep levels. Most bulk AIN crystals are semi-insulating and have below-band-gap absorption bands in the ultraviolet and visible (many of the crystals are colored to the eye). This indicates the presence of active point defects. Oxygen is the most common impurity and substitutes for nitrogen, thus behaving as a deep donor. ${ }^{4-8}$ Nitrogen vacancies ${ }^{9-11}$ are expected to be present and act as deep donors, while aluminum vacancies will be acceptors. ${ }^{12}$ Point defect complexes (e.g., a substitutional oxygen impurity ion with an adjacent aluminum vacancy or close pairs of nitrogen and aluminum vacancies) may also form during growth. Other deep levels may include transition-metal ions occupying aluminum (i.e., cation) sites. A central theme in the study of AlN is identifying all the possible charge states of native defects and impurities.

Generally speaking, all semiconductors contain point defects; their nature and their concentrations vary from one material to another and depend on a large number of factors. "Starting materials" used to grow the crystals may be a source of impurities, or the impurities may come from the growth atmosphere or the apparatus. Vacancies and 
interstitials can result from thermodynamic considerations, and even greater numbers of vacancies may be present to charge compensate the impurities. In some crystals, there is a tendency to grow nonstoichiometrically. Thus, it is not surprising that advanced semiconductors, especially in their early stages of development, have easily measured concentrations of point defects. In wide-band-gap semiconductors, these defects occur at levels varying from a few parts per billion to a few hundred parts per million.

\subsection{Zinc Oxide}

In recent years, considerable attention has been focused on the development of zinc oxide $(\mathrm{ZnO})$ for electro-optic applications. ${ }^{13-15}$ Among its significant advantages are (1) the relatively easy growth of large single crystals suitable for homoepitaxy and (2) a large exciton binding energy of about $60 \mathrm{meV}$. Zinc oxide has the potential to efficiently perform a wide variety of functions. When it is doped with the appropriate impurities, this material can be used to produce ultraviolet lasers and detectors. A more recent and new application of $\mathrm{ZnO}$ is in spintronics. ${ }^{16}$ This is an evolving field that uses the ferromagnetic properties of a semiconductor to control the spin of the carriers. For useful spintronic devices, the semiconductor must be ferromagnetic at room temperature. Preliminary reports in the literature suggest that Co and Mn may be good candidates to make $\mathrm{ZnO}$ ferromagnetic at elevated temperatures. ${ }^{17}$

Although $\mathrm{ZnO}$ is an excellent ultraviolet light emitter, ${ }^{18}$ its electro-optic applications are limited by the difficulties encountered when attempting to incorporate shallow p-type dopants. ${ }^{19,20}$ Most of the bulk $\mathrm{ZnO}$ crystals grown today are $\mathrm{n}$ type because they contain significant amounts of shallow donor impurities such as $\mathrm{Al}$ and $\mathrm{Ga}$, and perhaps hydrogen, zinc interstitials, and oxygen vacancies. Typical room-temperature electron concentrations are on the order of $1 \times 10^{17} \mathrm{~cm}^{-3}$. In some bulk crystals, transition-metal ions (e.g., $\mathrm{Ni}^{2+}, \mathrm{Fe}^{2+}, \mathrm{Mn}^{2+}$, and $\mathrm{Co}^{2+}$ ) are present at concentrations approaching a part per million and thus provide deeper donor levels. Success in $\mathrm{p}$ doping can only be achieved 
if the various unwanted donors are understood and minimized. Toward this end, the characteristic spectra that identify each donor must be determined using photoluminescence $(\mathrm{PL})$ and electron paramagnetic resonance $(\mathrm{EPR})$ techniques. Then, questions must be answered about the origins of the donor impurities and the mechanisms that lead to the formation of the native-defect donors. Also it is important to know if donors in $\mathrm{ZnO}$ can form complexes and become electrical and optically inactive.

There are a variety of acceptors that may be considered when attempting to produce p-type $\mathrm{ZnO}$. These include the Group IA and IB elements ( $\mathrm{Li}, \mathrm{Na}, \mathrm{Cu}, \mathrm{Ag}$ ), the Group V elements (N, P, and As), and zinc vacancies. It is now generally understood that the lithium and sodium ions (substituting on the cation sites) and the zinc vacancies form deep acceptor levels. In the case of the neutral alkali acceptors, the hole is primarily localized on only one of the neighboring oxygen ions ${ }^{21}$ and the accompanying significant lattice relaxation makes the acceptors very deep. A similar localization of the hole occurs for the singly ionized zinc vacancy. ${ }^{22}$ The neutral $\mathrm{Cu}^{2+}$ and $\mathrm{Ag}^{2+}$ acceptors (using ionic notation), with their $3 \mathrm{~d}^{9}$ and $4 \mathrm{~d}^{9}$ electron configurations, respectively, are also deep acceptors. $^{23}$ Recent work with nitrogen, where a donor-acceptor pair (DAP) recombination peak is observed near $3.24 \mathrm{eV}$, suggests that the neutral nitrogen acceptor ionization energy is approximately $205 \mathrm{meV} .^{24}$ Thus, the nitrogen acceptors are reasonably shallow in $\mathrm{ZnO}$ and are expected to provide conducting p-type material. However, incorporation of isolated nitrogen in thin films and bulk crystals has proven to be difficult and is presently the focus of many research groups. Phosphorus and arsenic possibly form shallow acceptors in $\mathrm{ZnO}$, but they have not yet been the subject of many studies. Difficulties may arise during the incorporation of phosphorus and especially arsenic because of their significantly larger ion size, compared to oxygen.

Beginning in the late 1960 s and extending into the early 1980 s, a series of studies of native defects in $\mathrm{ZnO}$ were reported. The first of these to be investigated was the singly ionized oxygen vacancy. Smith and Vehse ${ }^{25}$ produced oxygen vacancies in $\mathrm{ZnO}$ 
by electron irradiation and used EPR to examine the hyperfine pattern associated with the singly ionized charge state of the defect. They observed interactions with one axial zinc neighbor and three basal plane zinc neighbors, thus proving that they were monitoring oxygen vacancies. A slightly anisotropic g matrix was observed with principal values near 1.995. Other investigators have verified and extended these results. ${ }^{26,27}$ It is interesting to note that this EPR signal from the singly ionized oxygen vacancy has only been observed in particle-irradiated crystals, and not in material that is as-grown or thermally annealed. Although the $\mathrm{ZnO}$ literature contains many papers that invoke the presence of oxygen vacancies, there is little direct evidence to support these claims. Much of the confusion arises from the wrong assignment of the EPR signal at $g=1.96$ to singly ionized oxygen vacancies. ${ }^{28,29}$ This latter signal is due to shallow donors such as $\mathrm{Al}, \mathrm{Ga}, \mathrm{In}, \mathrm{H}$, and zinc interstitials. ${ }^{20,30-33}$

A second set of early studies focused on the zinc vacancy. ${ }^{22,34}$ These defects are paramagnetic in the singly ionized state. The hole is trapped on one of the four oxygen neighbors and forms a deep highly localized acceptor. This localization is verified by the anisotropy of the g matrix (its principal values range from 2.003 to 2.018). Electron irradiation was used to form these deep acceptors. Interestingly, it was also discovered that the neutral state of the zinc vacancy is paramagnetic and forms an $\mathrm{S}=1$ defect with the two holes trapped on separate nearest-neighbor oxygen ions. ${ }^{22}$

Optically detected magnetic resonance (ODMR) has been used to study shallow donors in $\mathrm{ZnO} .^{32,33}$ In these experiments, the intensity of the deep luminescence (donoracceptor recombination) is monitored while the sample is in a microwave cavity at low temperature. Transitions induced by the microwaves cause changes in the intensity of the emission when resonances occur as the magnetic field is swept through regions of interest. Direct evidence was obtained in these experiments for indium and gallium being shallow donors (i.e., their hyperfine patterns were observed). These ODMR signals occurred at a g value of 1.96. This provided clear evidence that the EPR signal at this $g$ 
value is due to shallow donors and free carriers, and is not due to oxygen vacancies. Additional recent ODMR experiments have also been reported. ${ }^{35-37}$

The green emission from $\mathrm{ZnO}$ continues to be a topic of major interest. ${ }^{38-42}$ It is easily observed in bulk crystals and in thin films produced by a variety of growth techniques. There is now an increasing awareness among investigators that there can be more than one contribution to the emission in the 500 to $520 \mathrm{~nm}$ region. ${ }^{35,43}$ In an early paper, Dingle ${ }^{23}$ described a detailed study of the broad structured green emission band peaking near $510 \mathrm{~nm}$ in $\mathrm{ZnO}$ and proved that it was due to copper impurities. He showed that the zero-phonon line associated with this emission is split because of the two isotopes of copper $\left({ }^{63} \mathrm{Cu}\right.$ and $\left.{ }^{65} \mathrm{Cu}\right)$ and he also showed that the Zeeman splitting of these no-phonon emissions gave g values in direct agreement with the EPR results reported by Dietz et al. ${ }^{44}$ for $\mathrm{Cu}^{2+}$ in $\mathrm{ZnO}$. This work conclusively showed that the structured green emission in $\mathrm{ZnO}$ is due to a localized excitation of an isolated $\mathrm{Cu}^{2+}$ ion (i.e., a short-lived transfer of an electron from a neighboring oxygen to the copper ion). Separate from this work, various groups in recent years have observed green emission from $\mathrm{ZnO}$ and have offered additional diverse models. Without identifying the acceptor, Reynolds et al. ${ }^{45}$ recently suggested that the structured green emission was transitions from shallow donors to a deep acceptor, and further suggested that a portion of the structure was caused by the presence of two distinct shallow donors. These investigators did not consider copper as an active participant because they had no evidence that copper was present in their sample. Other investigators have associated the green emission in $\mathrm{ZnO}$ with singly ionized oxygen vacancies. However, this suggested correlation must be questioned because of the mistaken assignment of the EPR signal at " $\mathrm{g}=1.96$ " to singly ionized oxygen vacancies. ${ }^{28,29}$ As mentioned earlier, the commonly observed EPR signal at $g=$ 1.96 is due to neutral shallow donors, while a different and only infrequently observed EPR signal with $g_{\|}=1.9945$ and $g_{\perp}=1.9960$ is due to singly ionized oxygen vacancies. ${ }^{25}$ 


\section{CHAPTER 2}

\section{EPR and ENDOR: Principles and Instrumentation}

\subsection{Electron Paramagnetic Resonance}

Magnetic resonance spectroscopy has proven to be a valuable experimental technique in many areas of science. Nuclear magnetic resonance (NMR) is one such technique with applications in fields as diverse as biology, chemistry, geology, and materials science. An equally useful form of magnetic resonance spectroscopy is electron paramagnetic resonance (EPR). Although not as widely available as NMR, EPR is a very powerful technique used to conduct basic research in disciplines such as physics, chemistry, and materials research. NMR uses nuclear magnetic moments to absorb rf energy, while EPR uses unpaired electron spins to absorb microwave energy.

EPR has been extensively developed over the many years since its inception. In 1896, Zeeman first discovered the separation of energy levels in a magnetic field. Later in 1922, Stern and Gerlach showed that the magnetic moment of an electron in a magnetic field can have only two orientations. In 1925, Uhlenbeck and Goudsmit introduced the idea of electron spin to describe the magnetic moment. This moment has discrete values and gives two energy levels for an electron. These energy levels are degenerate in the absence of a magnetic field, but this degeneracy is lifted in the presence of a magnetic field. In recognition of Zeeman's discovery, the splitting of energy levels in a magnetic field is called Zeeman splitting. In the 1940s, EPR experiments were conducted by Zavoisky (Soviet Union), Cummerow and Holliday (USA), and Bleaney and Penrose (England.) Since then, many refinements and improvements have been made in EPR spectrometers, but the general principles of how it works remain the same.

The most important limitation of EPR is the requirement of a net spin angular momentum (i.e., one or more unpaired spins). Fortunately, there are many samples in solid, liquid, and even gaseous form that satisfy this requirement. The present dissertation deals with point defects in crystalline environments. A variety of point 
defects in crystals contain an unpaired spin, and in nearly every case the defects are either paramagnetic before illumination or convert to a paramagnetic state as a result of illumination at sufficiently low temperatures. Some common examples of point defects containing unpaired electrons are vacancies, antisites, and transition-metal impurities.

\subsubsection{Basic Principles of EPR}

A description of EPR begins with the magnetic moment of an electron in a static magnetic field. The energy of a magnetic dipole in a magnetic field is defined as

$$
\mathrm{E}=-\boldsymbol{\mu} \cdot \mathbf{B}
$$

where $\mu$ is the magnetic moment and $\mathrm{B}$ is the magnetic field. If the $\mathrm{z}$ axis is taken to be along $\mathrm{B}$, then the component of the electron spin magnetic moment is

$$
\mu_{\mathrm{z}}=-\beta \mathrm{gm}_{\mathrm{s}}
$$

where $\beta$ is the Bohr magneton, $\mathrm{m}_{\mathrm{S}}$ is the component of spin along the $\mathrm{z}$ axis, and $\mathrm{g}$ is 2.00232 for a free electron. The negative sign is due to the negative charge of an electron. Substituting Eq. (2.2) into Eq. (2.1) yields

$$
\mathrm{E}=\beta \mathrm{gBm}_{\mathrm{S}} .
$$

Here, quantum mechanics dictates that $\mathrm{m}_{\mathrm{S}}$ takes quantized values of $\mathrm{S}, \mathrm{S}-1, \mathrm{~S}-2, \ldots$, $-\mathrm{S}+1,-\mathrm{S}$. For a system of spin angular momentum $\mathrm{S}=1 / 2$ (i.e., a free electron), the two values of $m_{S}$ are $+1 / 2$ (spin up) and $-1 / 2$ (spin down). Thus, the Zeeman energy levels are given by the following expressions.

$$
\begin{aligned}
& \mathrm{E}_{+}=\frac{1}{2} \beta \mathrm{gB} \\
& \mathrm{E}_{-}=-\frac{1}{2} \beta \mathrm{gB}
\end{aligned}
$$

EPR transitions can be induced in this $\mathrm{S}=1 / 2$ system by exposing the unpaired electron to electromagnetic radiation with photon energy hv that matches the separation between the two energy levels. This resonance condition can be written as

$$
\mathrm{h} v=\Delta \mathrm{E}=\beta \mathrm{gB} .
$$




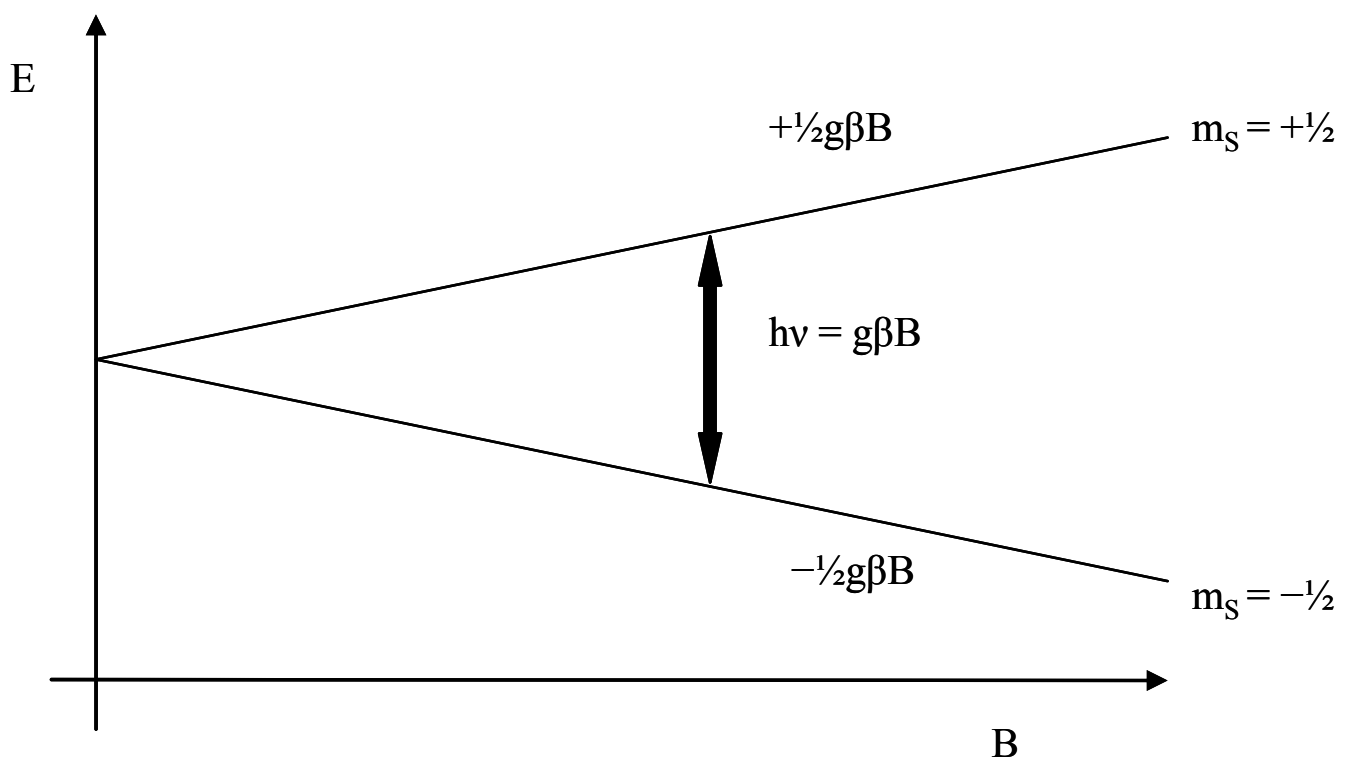

Figure 2.1. Energy level splitting for an $S=1 / 2$ system in an increasing magnetic field. The vertical arrow represents the resonance position.

Many spectroscopic techniques vary the frequency of the excitation source. For EPR, however, the excitation frequency remains constant while the energy separation of the two states is varied by changing the field. The resonance frequency of the microwave cavity is fixed and cannot be easily changed, thus, sweeping the magnetic field is a practical solution. Most spin systems of interest will have a $\mathrm{g}$ factor close to that of a free electron. Many EPR spectrometers operate at $\mathrm{X}$ band (i.e., $\sim 10 \mathrm{GHz}$ microwaves), giving $\mathrm{ag}=2$ resonance at around 3400 Gauss. Figure 2.1 shows how the energy levels vary with increasing magnetic field. The vertical arrow in Fig. 2.1 represents the EPR transition that occurs at the field position corresponding to the resonance condition. In general, EPR transitions have $\Delta \mathrm{m}_{\mathrm{S}}= \pm 1$ selection rules. When $\mathrm{S}$ is greater than $1 / 2$ it is also possible to have "forbidden" transitions where $\Delta \mathrm{m}_{\mathrm{S}}= \pm 2$.

For isolated spin systems, a spin-Hamiltonian is used to describe the individual spin states. Eigenvalues and eigenvectors are extracted from the spin-Hamiltonian to obtain transition energies and transition probabilities. The spin-Hamiltonian describes 
interactions with the applied magnetic field, the crystal field, and nuclear magnetic and electric quadrupole moments. Thus far in this section, only the spin-Hamiltonian for a free electron has been considered. In a material, the orbital angular momentum of the electron must also be included in the electron-Zeeman term as it is the orbital angular momentum that produces deviations of the $\mathrm{g}$ factor from that of the free spin value.

The electron-Zeeman term contains both the interaction between the applied magnetic field and the magnetic dipole associated with the spin and the spin-orbit coupling interaction of the spin and the orbital momentum. A general expression for the electron-Zeeman term has been derived by Weil, Bolton, and Wertz, ${ }^{46}$ and is briefly described below.

$$
\begin{aligned}
& \hat{H}_{E Z}=\hat{H}_{M A G}+\hat{H}_{S O} \\
& \hat{H}_{M A G}=\beta \mathbf{B} \cdot\left(\hat{\mathbf{L}}+\mathrm{g}_{\mathrm{e}} \hat{\mathbf{S}}\right) \\
& \hat{H}_{S O}=\lambda(\hat{\mathbf{L}} \cdot \hat{\mathbf{S}})
\end{aligned}
$$

$\mathbf{B}$ is the applied magnetic field

$\hat{\mathbf{S}}$ is the spin angular momentum operator

$\hat{\mathbf{L}}$ is the orbital angular momentum operator

$\lambda$ is the spin-orbit coupling parameter

Substituting Eqs. (2.7) and (2.8) into Eq. (2.6) gives

$$
\hat{H}_{E Z}=\beta \mathbf{B} \cdot\left(\hat{\mathbf{L}}+\mathrm{g}_{\mathrm{e}} \hat{\mathbf{S}}\right)+\lambda(\hat{\mathbf{L}} \cdot \hat{\mathbf{S}})=\beta \mathbf{B} \cdot \overrightarrow{\mathbf{g}} \cdot \hat{\mathbf{S}} .
$$

This expresses the electron-Zeeman term as a single term with a g matrix defined as

$$
\overrightarrow{\mathbf{g}}=\mathrm{g}_{\mathrm{e}} \mathbf{I}+2 \lambda \Lambda=\left(\begin{array}{lll}
g_{x x} & g_{x y} & g_{x z} \\
g_{y x} & g_{y y} & g_{y z} \\
g_{z x} & g_{z y} & g_{z z}
\end{array}\right) \text {. }
$$

Here, the $\mathrm{g}$ matrix is symmetric (i.e., $\mathrm{g}_{\mathrm{ij}}=\mathrm{g}_{\mathrm{ji}}$ ), and $\Lambda$ accounts for any anisotropy arising from the orbital angular momentum. The symmetry of the g matrix reduces the number of independent parameters to six. When written in its principal axis system, these are the three principal $g$ values (i.e., the $g$ values along the principal axes $x_{g}, y_{g}$, and $z_{g}$ ) and three 
Euler angles that define a rotation of the $\mathrm{g}$ matrix to another coordinate system. The $\mathrm{g}$ matrix can be written in coordinate systems other than its principal system. In these cases, the g matrix will have the form presented in Eq. (2.10) where the six parameters are the three diagonal and the three independent off-diagonal terms.

For a spin system with $S=1 / 2, L=0$, the basis states $| \pm 1 / 2\rangle$ are the eigenstates and the Pauli spin matrices are written below.

$$
\begin{aligned}
\hat{\mathbf{S}}_{\mathbf{x}} & =\left(\begin{array}{ll}
0 & 1 \\
1 & 0
\end{array}\right) \\
\hat{\mathbf{S}}_{\mathbf{y}} & =\left(\begin{array}{cc}
0 & -i \\
i & 0
\end{array}\right) \\
\hat{\mathbf{S}}_{\mathbf{z}} & =\left(\begin{array}{cc}
1 / 2 & 0 \\
0 & -1 / 2
\end{array}\right)
\end{aligned}
$$

Planck's constant has been left out for simplicity. For $\mathrm{L}=0$, there is no spin-orbit coupling and no interaction of the orbital angular momentum with the applied magnetic field. Thus, by setting $\hat{\mathbf{L}}=0$, the electron-Zeeman term in the spin-Hamiltonian becomes

$$
\hat{H}_{\mathrm{EZ}}=\beta \mathrm{g}_{\mathrm{e}} \mathbf{B} \cdot \hat{\mathbf{S}}
$$

This also means that there is no anisotropy since $\Lambda=0$, and all the principal values of the g matrix have the value $g_{\mathrm{e}}$. Expanding Eq. (2.14) gives

$$
\hat{H}_{\mathrm{EZ}}=\beta \mathrm{g}_{\mathrm{e}}\left(\mathrm{B}_{\mathrm{x}} \mathbf{S}_{\mathbf{x}}+\mathrm{B}_{\mathrm{y}} \mathbf{S}_{\mathbf{y}}+\mathrm{B}_{\mathrm{z}} \mathbf{S}_{\mathrm{z}}\right) .
$$

If a coordinate system is defined with the $\mathrm{z}$ axis along the field $\left(\mathrm{B}_{\mathrm{z}}=\mathrm{B}, \mathrm{B}_{\mathrm{x}}=\mathrm{B}_{\mathrm{y}}=0\right)$, then combining Eqs. (2.13) and (2.15) gives

$$
\hat{H}_{\mathrm{EZ}}=\beta \mathrm{g}_{\mathrm{e}} \mathrm{BS}_{\mathrm{z}}=\left(\begin{array}{cc}
\frac{1}{2} \beta \mathrm{g}_{\mathrm{e}} \mathrm{B} & 0 \\
0 & -\frac{1}{2} \beta \mathrm{g}_{\mathrm{e}} \mathrm{B}
\end{array}\right) .
$$

The energy eigenvalues of this spin-Hamiltonian are $\frac{1}{2} \beta g_{\mathrm{e}} \mathrm{B}$ for the spin up state and $-\frac{1}{2} \beta g_{\mathrm{e}} \mathrm{B}$ for the spin down state.

For a case where $\mathrm{L}$ is nonzero, the orbital angular momentum produces a deviation from the free spin value $g_{e}$ and an anisotropic g matrix. Expressing the electronZeeman term in the principal axis system of the $\mathrm{g}$ matrix , the spin-Hamiltonian becomes 


$$
\hat{H}_{\mathrm{EZ}}=\beta \mathbf{B} \cdot\left(\begin{array}{ccc}
\mathrm{g}_{\mathrm{xx}} & 0 & 0 \\
0 & \mathrm{~g}_{\mathrm{yy}} & 0 \\
0 & 0 & \mathrm{~g}_{\mathrm{zz}}
\end{array}\right) \cdot \hat{\mathbf{s}} .
$$

Upon expanding, the spin-Hamiltonian is similar to Eq. (2.15).

$$
\hat{H}_{\mathrm{EZ}}=\beta\left(\mathrm{B}_{\mathrm{x}} \mathrm{g}_{\mathrm{xx}} \mathbf{S}_{\mathbf{x}}+\mathrm{B}_{\mathrm{y}} \mathrm{g}_{\mathrm{yy}} \mathbf{S}_{\mathbf{y}}+\mathrm{B}_{\mathrm{z}} \mathrm{g}_{\mathrm{zz}} \mathbf{S}_{\mathrm{z}}\right)
$$

For an axial $\mathrm{g}$ matrix $\left(\mathrm{g}_{\mathrm{xx}}=\mathrm{g}_{\mathrm{yy}}=\mathrm{g}_{\perp}\right.$ and $\left.\mathrm{g}_{\mathrm{zz}}=\mathrm{g}_{\|}\right)$, an effective $\mathrm{g}$ value ( $\left.\mathrm{g}_{\text {eff }}\right)$ is defined by the following expression.

$$
\mathrm{g}_{\text {eff }}^{2}=\mathrm{g}_{\|}^{2} \cos ^{2} \theta+\mathrm{g}_{\perp}^{2} \sin ^{2} \theta
$$

Here, the angle $\theta$ represents the angle that the magnetic field makes with the unique axis of the g matrix, i.e., the magnetic field is parallel to the unique axis when $\theta=0^{\circ}$ and is perpendicular to the unique axis when $\theta=90^{\circ}$.

There are many paramagnetic systems with $\mathrm{S}>1 / 2$. For example, transitionmetal-ion impurities (such as $\mathrm{Fe}, \mathrm{Cr}, \mathrm{Mn}$, etc.) are often present in a paramagnetic state with $S>1 / 2$ in a variety of insulator and semiconductor crystals. Fine structure splittings occur for spin systems with $S \geq 1$. In the spin-Hamiltonian, this is called the fine structure or crystal field term. This splitting arises when the crystalline electric field lifts the degeneracies of the $\mathrm{d}$ electrons in a transition-metal ion or when two unpaired electrons on neighboring ions experience dipole-dipole interactions. For an $\mathrm{S}=1$ spin system, the fine structure term in the spin-Hamiltonian can be written as

$$
\hat{H}_{\mathrm{FS}}=\hat{\mathbf{S}} \cdot \overrightarrow{\mathbf{D}} \cdot \hat{\mathbf{S}} .
$$

Like the $g$ matrix, the $D$ matrix can be written with 3 principal values $\left(D_{x x}, D_{y y}\right.$, and $\left.D_{z z}\right)$ and three Euler angles. However, the D matrix is traceless (i.e., the sum of its diagonal elements is zero). The principal values of the $\mathrm{D}$ matrix can be express by two parameters using the following expressions

$$
\begin{aligned}
& D_{x x}=-\frac{1}{3} D+E \\
& D_{y y}=-\frac{1}{3} D-E \\
& D_{z z}=\frac{2}{3} D
\end{aligned}
$$


where D describes the axially symmetric component and $\mathrm{E}$ arises from the fact that the $\mathrm{x}$ and y directions are not equivalent. Equation (2.20) can be written in the following form.

$$
\hat{H}_{\mathrm{FS}}=\mathrm{D}_{\mathrm{xx}} \mathbf{S}_{\mathbf{x}}^{2}+\mathrm{D}_{\mathrm{yy}} \mathbf{S}_{\mathbf{y}}^{2}+\mathrm{D}_{\mathrm{zz}} \mathbf{S}_{\mathbf{z}}^{2}=\mathrm{D}\left[\mathbf{S}_{\mathbf{z}}^{2}-\frac{1}{3} \mathrm{~S}(\mathrm{~S}+1)\right]+\mathrm{E}\left(\mathbf{S}_{\mathbf{x}}^{2}+\mathbf{S}_{\mathbf{y}}^{2}\right)
$$

Since there is no dependence on the magnetic field strength, the fine structure term in the spin-Hamiltonian produces a splitting when no external field is present. This effect is referred to as "zero-field splitting." As an example, Fig. 2.2 shows the energy levels vs. magnetic field for an $\mathrm{S}=1$ spin system. For many $\mathrm{d}$ and f electron systems in transitionion-metal and rare-earth ions, the Hamiltonian given in Eq. (2.20) is not sufficient, and higher order terms are required to explain observed EPR spectra. For these spin systems, the crystal field term of the spin-Hamiltonian is written using crystal field operators.

$$
\hat{H}_{\mathrm{CF}}=\sum_{l=\text { even }} \sum_{m=-l}^{l} B_{l}^{m} O_{l}^{m}
$$

Here, $B_{l}^{m}$ are constants and the $O_{l}^{m}$ are crystal field operators. Stevens operators are a widely used form of the crystal field operators.

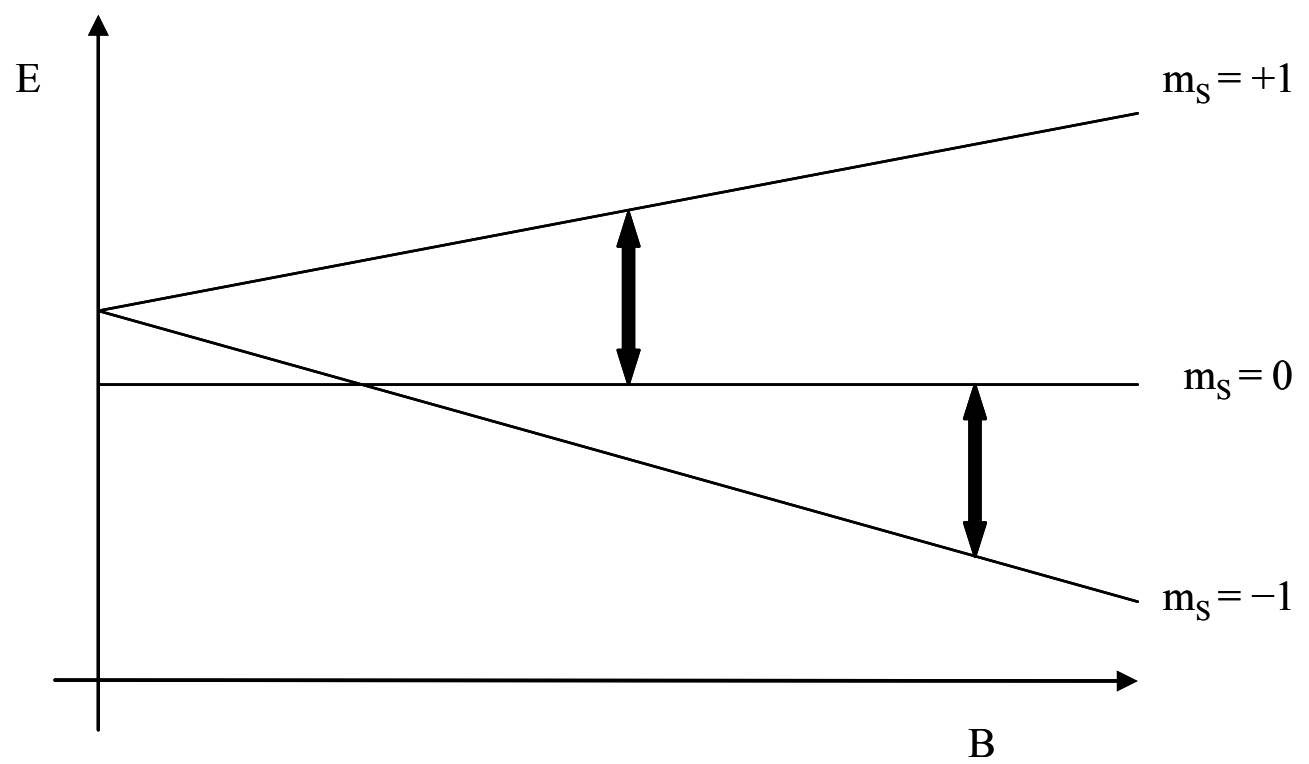

Figure 2.2. Energy level diagram of an $\mathrm{S}=1$ spin system. Solid vertical lines represent the allowed transitions $\left(\Delta \mathrm{m}_{\mathrm{S}}= \pm 1\right)$. 


\subsubsection{Hyperfine Interactions with Nuclei}

Thus far in this Chapter, only electrons have been discussed (i.e., free spins, anisotropic g matrices, and multiple interacting spins). Now, interactions of the unpaired spins with nuclear magnetic moments are considered. Nuclear magnetic moments are described by a nuclear spin I, just as electron magnetic moments are described by an electron spin S. Some elements have isotopes with different values of I. Nuclear magnetic moments quantize along the magnetic field, giving rise to discrete states with quantum numbers $\mathrm{m}_{\mathrm{I}}=\mathrm{I}, \mathrm{I}-1, \ldots, 1-\mathrm{I},-\mathrm{I}$. These nuclear moments lead to splittings in the EPR spectra as a result of overlap of the wave function at the nucleus, dipole-dipole interactions, and interactions with electric field gradients. If the unpaired electron spins interact with the central nucleus (as the case with impurities), the interaction is referred to as hyperfine. For interactions with one or more neighboring nuclei, the effects are called superhyperfine. In many cases, superhyperfine interactions are referred to as hyperfine.

The hyperfine and superhyperfine interactions add additional terms to the spin-

Hamiltonian. For an electron spin S and one nuclear spin I, the hyperfine term appears as

$$
\hat{H}_{\mathrm{HF}}=\hat{\mathbf{I}} \cdot \overrightarrow{\mathbf{A}} \cdot \hat{\mathbf{S}}
$$

where $\hat{\mathbf{I}}$ is the nuclear spin operator and $\overrightarrow{\mathbf{A}}$ is the hyperfine matrix describing the interactions between the magnetic moments. For isotropic $g$ and hyperfine matrices with $\mathrm{S}=1 / 2$ and $\mathrm{I}=1 / 2$, the energy eigenvalues of the Hamiltonian are expressed as

$$
\mathrm{E}\left(\mathrm{m}_{\mathrm{S}}, \mathrm{m}_{\mathrm{I}}\right)=\beta \mathrm{gBm_{ \textrm {S } }}+\mathrm{Am}_{\mathrm{S}} \mathrm{m}_{\mathrm{I}} .
$$

Substituting the possible values of the electron and nuclear spin quantum numbers into Eq. (2.27) gives the following expressions for the resulting four energy levels.

$$
\begin{aligned}
& \mathrm{E}(1 / 2,1 / 2)=\frac{1}{2} \beta \mathrm{gB}+\frac{1}{4} \mathrm{~A} \\
& \mathrm{E}(1 / 2,-1 / 2)=\frac{1}{2} \beta \mathrm{gB}-\frac{1}{4} \mathrm{~A} \\
& \mathrm{E}(-1 / 2,1 / 2)=-\frac{1}{2} \beta \mathrm{gB}-\frac{1}{4} \mathrm{~A} \\
& \mathrm{E}(-1 / 2,-1 / 2)=-\frac{1}{2} \beta g B+\frac{1}{4} \mathrm{~A}
\end{aligned}
$$


Here, A has units of MHz; in other words, the energies $\mathrm{E}$ have been divided by Planck's constant. Figure 2.3 shows how an $S=1 / 2$ spin system evolves into an $S=1 / 2, I=1 / 2$ spin system. The dashed lines in this figure are similar to those found in Fig. 2.1, while the solid lines illustrate the effects of the nuclear spin. There are selection rules that determine which transitions are allowed and forbidden in this four-level spin system. For $\mathrm{S}=1 / 2$ and $\mathrm{I}=1 / 2$, the selections rules are $\Delta \mathrm{m}_{\mathrm{S}}= \pm 1, \Delta \mathrm{m}_{\mathrm{I}}=0$ for allowed EPR transitions, and $\Delta \mathrm{m}_{\mathrm{S}}= \pm 1, \Delta \mathrm{m}_{\mathrm{I}}= \pm 1$ for forbidden EPR transitions.

The hyperfine matrix $\overrightarrow{\mathbf{A}}$ has two contributions, an isotropic part $(a \overrightarrow{\mathbf{I}})$ and an anisotropic part $(\overrightarrow{\mathbf{B}})$. The hyperfine matrix can be written as

$$
\overleftrightarrow{\mathbf{A}}=a \overleftrightarrow{\mathbf{I}}+\overleftrightarrow{\mathbf{B}}
$$

where $\overrightarrow{\mathbf{I}}$ is the identity matrix. The isotropic part $a$ is the Fermi-contact term that arises

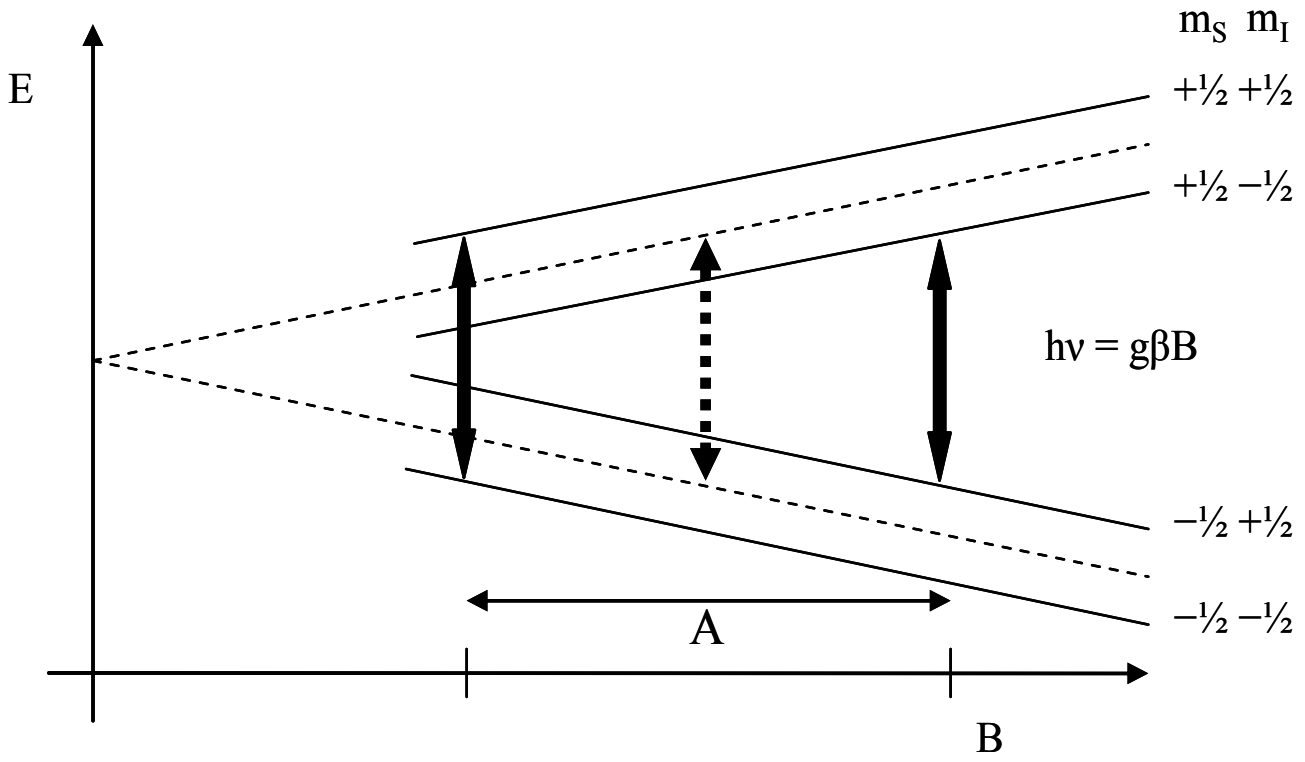

Figure 2.3. Energy level diagram of an $\mathrm{S}=1 / 2, \mathrm{I}=1 / 2$ system. Solid lines represent the energy levels that are split by the hyperfine interaction. Dashed lines represent the energy levels in the absence of a hyperfine interaction. The solid and dashed vertical arrows represent EPR transitions with and without hyperfine interactions, respectively. 
from the overlap of the electron wave function onto the nucleus. The Fermi-contact term can be written as

$$
a=\frac{2}{3} \mu_{0} \mu_{e} \mu_{N}|\psi(\mathbf{r})|^{2}
$$

where $\mu_{\mathrm{e}}$ is the electron magnetic moment, $\mu_{\mathrm{N}}$ is the nuclear magnetic moment, and $\mathrm{r}$ is the position (i.e., the location) of the nucleus. This Fermi-contact term depends directly on the unpaired electron spin density at the nucleus. For example, if the unpaired spin were in a p orbital there would be no unpaired spin density at the nucleus and thus $a$ would be zero. In contrast, an unpaired spin in an s orbital has a large unpaired spin density at the nucleus and thus $a$ is large.

The anisotropic part $\overrightarrow{\mathbf{B}}$ represents the dipole-dipole interaction of the electron magnetic moment and the nuclear magnetic moment. The matrix $\overrightarrow{\mathbf{B}}$ can be written in its principal axis system as a traceless matrix of the form

$$
\overrightarrow{\mathbf{B}}=\left(\begin{array}{ccc}
-\mathrm{b}-\mathrm{b}^{\prime} & 0 & 0 \\
0 & -\mathrm{b}+\mathrm{b}^{\prime} & 0 \\
0 & 0 & 2 \mathrm{~b}
\end{array}\right)
$$

where $b$ represents the primary dipole-dipole interaction and b' represents any deviation from axial symmetry. The elements of $\overrightarrow{\mathbf{B}}$ can be written in general as

$$
\mathrm{B}_{\mathrm{ij}}=\frac{\mu_{0}}{4 \pi} \mathrm{g}_{\mathrm{e}} \mu_{\mathrm{B}} \mathrm{g}_{\mathrm{N}} \mu_{\mathrm{N}} \int\left(\frac{3 \mathrm{x}_{\mathrm{i}} \mathrm{x}_{\mathrm{j}}}{\mathrm{r}^{5}}-\frac{\delta_{\mathrm{ij}}}{\mathrm{r}^{3}}\right)|\psi(r)|^{2} d V
$$

From the experimentally determined principal values of the $\overrightarrow{\mathbf{B}}$ matrix, values for the $b$ and b' parameters can be extracted.

$$
\begin{aligned}
& \mathrm{b}=\frac{1}{2} \mathrm{~B}_{\mathrm{zz}} \\
& \mathrm{b}^{\prime}=\frac{1}{2}\left(\mathrm{~B}_{\mathrm{yy}}-\mathrm{B}_{\mathrm{xx}}\right)
\end{aligned}
$$

These parameters, $b$ and b', yield information on the spatial distribution of the electronic wave function, and also yield information regarding the locations of interacting nuclei.

Nuclear magnetic moments due to spin angular momentum should be considered much like the angular momenta of electrons. In EPR, a nuclear spin interacts with an 
applied magnetic field and usually only shifts the electron-spin energy levels rather than split them further. This is the nuclear-Zeeman interaction, and it is considerably smaller that the electron-Zeeman interaction (approximately three orders of magnitude smaller). Analogous to the electron, the nucleus also has a $g$ factor; however, since there is no orbital angular momentum equivalent for a nucleus, the nuclear $g$ factor is normally isotropic. Thus, the nuclear-Zeeman term is written as

$$
\hat{H}_{\mathrm{NZ}}=-\mathrm{g}_{\mathrm{N}} \beta_{\mathrm{N}} \mathbf{B} \cdot \hat{\mathbf{I}} \text {. }
$$

The value of the nuclear $\mathrm{g}$ value $\left(\mathrm{g}_{\mathrm{N}}\right)$ is a defining property of a nucleus and can be easily obtained from tables in the literature. As will be seen later in this work, this information can be extremely useful in identification of nearby nuclei. Different magnetic isotopes of the same element will have different nuclear g values. This information, when coupled with the knowledge of the relative ratios of isotopes, can be used for further identification of an interacting nucleus.

There is one last term of the spin Hamiltonian needed to fully describe hyperfine patterns in EPR and electron-nuclear double resonance (ENDOR) spectra. Just as an electron spin system with $\mathrm{S}>1 / 2$ has a crystal field interaction (fine structure), a nucleus with I $>1 / 2$ will interact with electric field gradients through the nuclear quadrupole matrix $\overrightarrow{\mathbf{Q}}$. The quadrupole term of the spin Hamiltonian is given by

$$
\hat{H}_{\mathrm{Q}}=\hat{\mathbf{I}} \cdot \overrightarrow{\mathbf{Q}} \cdot \hat{\mathbf{I}}
$$

where $\overrightarrow{\mathbf{Q}}$ can be written as a traceless matrix in its principal axis system.

$$
\overrightarrow{\mathbf{Q}}=\left(\begin{array}{ccc}
-\mathrm{q}+\mathrm{q}^{\prime} & 0 & 0 \\
0 & -\mathrm{q}-\mathrm{q}^{\prime} & 0 \\
0 & 0 & 2 \mathrm{q}
\end{array}\right)
$$

As with the anisotropic part of the hyperfine, q' represents deviations from axial symmetry. The quadrupole contribution causes shifts in the energy levels that are small in comparison to the electron-Zeeman effects and are usually not detectable in EPR spectra. For this reason, the quadrupole information is typically gathered from ENDOR 
experiments. The ENDOR technique is described in Section 2.2.

All the terms of the spin Hamiltonian necessary to describe a typical spin system have now been presented. The full Hamiltonian appears as

$$
\hat{H}=\beta \mathbf{B} \cdot \overrightarrow{\mathbf{g}} \cdot \hat{\mathbf{S}}+\hat{\mathbf{S}} \cdot \overrightarrow{\mathbf{D}} \cdot \hat{\mathbf{S}}+\hat{\mathbf{I}} \cdot \overrightarrow{\mathbf{A}} \cdot \hat{\mathbf{S}}+\hat{\mathbf{I}} \cdot \overrightarrow{\mathbf{Q}} \cdot \hat{\mathbf{I}}-\mathrm{g}_{\mathrm{N}} \beta_{\mathrm{N}} \mathbf{B} \cdot \hat{\mathbf{I}}
$$

This spin-Hamiltonian includes only one nucleus. Sometimes there are two or more interacting nuclei present. The hyperfine splitting may not be the same for each nucleus, and the spin of the nuclei may not even be the same. For systems where the unpaired electron spin interacts with multiple nuclei, each nucleus will have separate nuclearZeeman, hyperfine, and electric quadrupole terms added to the Hamiltonian. In many cases, the hyperfine splittings of nuclei are not resolved in EPR and, as discussed later, the hyperfine information must be obtained from ENDOR data.

\subsubsection{EPR Transitions and Probabilities}

The spin Hamiltonian has been fully discussed and can now be used to examine the probabilities and selection rules of transitions. EPR transitions have two requirements, an external applied magnetic field to lift the degeneracy of the unpaired spin, and an oscillating magnetic field (i.e., microwave photons) to drive the transitions between the Zeeman-split levels. If the oscillating field is perpendicular to the applied static field, then the resulting field at the sample is

$$
\mathbf{B}=\mathrm{B}_{0} \hat{\mathbf{z}}+\mathrm{B}_{1} \cos \omega \mathrm{t} \hat{\mathbf{x}}
$$

where $\mathrm{B}_{0}$ is the static field, $\mathrm{B}_{1}$ is the oscillating field, and $\omega$ is the angular frequency of

the oscillating field. Substituting the field from Eq. (2.42) into a simple spin Hamiltonian with only an electron-Zeeman term gives

$$
H=g \beta B_{0} \mathbf{S}_{\mathbf{z}}+\mathrm{g} \beta \mathrm{B}_{1} \cos \omega \mathrm{t} \mathbf{S}_{\mathbf{x}} .
$$

Usually, the first term due to the static field is much greater than the second term. For any system where this is true, the affect of the oscillating field can be taken as a 
perturbation and Fermi's golden rule can be applied to determine the probabilities of transitions between spin up and spin down states. The probability is then expressed as

$$
W_{+1 / 2 \leftrightarrow-1 / 2} \propto\left|\left\langle+1 / 2\left|\hat{\mathbf{S}}_{\mathbf{x}}\right|-1 / 2\right\rangle\right|^{2}=1
$$

For the case where the oscillating field is perpendicular to the static field, the probability is one and the EPR spectrum has maximum intensity. Now consider the case where the oscillating field is applied parallel to the static field. Fermi's golden rule gives

$$
W_{+1 / 2 \leftrightarrow-1 / 2} \propto\left|\left\langle+1 / 2\left|\hat{\mathbf{S}}_{\mathbf{z}}\right|-1 / 2\right\rangle\right|^{2}=0
$$

and there would be no transitions. Note that the probability is the same for transitions from $-1 / 2$ to $+1 / 2$ (absorption) or $+1 / 2$ to $-1 / 2$ (stimulated emission). The selection rules are then determined from these applications of Fermi's golden rule. The eigenstates of the spin Hamiltonian may not be the simple spin states, instead the eigenstates may be linear combinations of the spin states. When this mixing occurs, "forbidden" transitions may become observable.

Since the probability of absorption is the same as stimulated emission, there must be a population difference between the two involved spin states in order to observe a transition. Typically, cooling the system using liquid helium or liquid nitrogen will enhance this population difference, as the lower energy state becomes more populated. At and near liquid helium temperatures, the spin-lattice relaxation times need to be taken into consideration. These are the characteristic times that it takes for an electron excited to an upper spin state to return to its ground state by emitting one or more phonons. If the temperature is too low, the spin-lattice relaxation time becomes very long and disrupts the population difference. The same effect can occur if the microwave power is too large, as upward transitions will be induced faster than the electrons can relax to their ground state. Optimizing an EPR signal requires adjusting both the temperature and the microwave power to maximize intensity. 


\subsection{Electron-Nuclear Double Resonance}

EPR often provides a large amount of information about point defects. However, there are cases where even EPR is limited in the information that can be obtained. The $\mathrm{g}$ matrix and the crystal field parameters are usually obtained fairly well with EPR. Hyperfine, quadrupole, and the identity of the defect sometimes cannot be obtained from EPR. The identity of the defect can be made from hyperfine splittings, if there are isotopes of measurable abundance present. This is done by comparing the abundance ratios to the ratios of line intensities of the observed spectra. Often, the nuclear hyperfine interactions are small compared to the EPR line width and the splittings cannot be resolved in EPR experiments. Also, the quadrupole effect is not present in first-order in EPR. To obtain these types of information, the electron-nuclear double resonance (ENDOR) technique was developed. ENDOR spectra can provide information that typical EPR spectra cannot, including the identity of neighboring nuclei, and thus a model of the defect.

ENDOR was developed by Feher in 1956. With ENDOR, identification can be made by determining the nuclear spin I and by measuring the nuclear $g$ value. In EPR, quadrupole effects give rise to only second order effects, but in ENDOR the quadrupole interaction is a first-order effect and produces splittings in the spectra, in much the same way as the crystal field splits EPR signals. ENDOR also utilizes an oscillating magnetic field perpendicular to the static magnetic field, however instead of using microwave frequencies, $\mathrm{rf}$ frequencies in the range of 1 to $100 \mathrm{MHz}$ are often used. Since the oscillating field is perpendicular to the static field, the ENDOR selection rules for a simple $\mathrm{S}=1 / 2, \mathrm{I}=1 / 2$ system are $\Delta \mathrm{m}_{\mathrm{S}}=0, \Delta \mathrm{m}_{\mathrm{I}}= \pm 1$. Unlike EPR, for ENDOR the applied static field remains constant while the rf frequency is swept across a preset range.

For the simple example of an $\mathrm{S}=1 / 2, \mathrm{I}=1 / 2$ system, the spin Hamiltonian is

$$
\hat{H}=\beta \mathbf{B} \cdot \overrightarrow{\mathbf{g}} \cdot \hat{\mathbf{S}}+\hat{\mathbf{I}} \cdot \overrightarrow{\mathbf{A}} \cdot \hat{\mathbf{S}}-\mathrm{g}_{\mathrm{N}} \beta_{\mathrm{N}} \mathbf{B} \cdot \hat{\mathbf{I}} .
$$

Taking the field along the $\mathrm{z}$ axis and assuming the matrices are isotropic, the spinHamiltonian becomes 


$$
\hat{H}=\beta g B \hat{\mathbf{S}}_{\mathbf{z}}+\mathrm{A}_{\mathbf{\mathbf { I }}} \hat{\mathbf{S}}_{\mathbf{z}}-v_{\mathrm{N}} \hat{\mathbf{I}}_{\mathbf{z}} .
$$

Here, $g_{N} \beta_{N} B$ is replaced with the nuclear frequency $v_{N}$, also commonly called the free nuclear spin value. The resulting energy levels are

$$
\mathrm{E}\left(\mathrm{m}_{\mathrm{S}}, \mathrm{m}_{\mathrm{I}}\right)=\beta \mathrm{gBm}_{\mathrm{S}}+\mathrm{Am}_{\mathrm{S}} \mathrm{m}_{\mathrm{I}}-v_{\mathrm{N}} \mathrm{m}_{\mathrm{I}}
$$

There are four possible combinations of the two values of $\mathrm{m}_{\mathrm{S}}$ and the two values of $m_{I}$. The relative positions of the energy levels depend on the values of $A / 2$ and $v_{N}$. Figure 2.4 shows the case where $\mathrm{A} / 2>v_{\mathrm{N}}$. Following the selection rules, there are two EPR transitions and two ENDOR transitions. For this case, the two ENDOR transitions are centered around A/2 and separated by $2 v_{\mathrm{N}}$. Thus, by measuring $2 v_{\mathrm{N}}$ for this system, the responsible nucleus can be identified. For $v_{N}>A / 2$ the order of energy levels changes. The diagram for this scenario is shown in Figure 2.5. Again, there are two EPR and two ENDOR transitions, but the difference is that the ENDOR lines are now centered on $v_{N}$ and separated by A. As before, the value of the free spin $v_{N}$ can be measured to make an identification of the responsible nucleus.

Even though ENDOR is a detection of nuclear transitions, it is not a direct observation. ENDOR transitions are observed by monitoring the EPR signal associated with the nucleus. The first step of this process is to saturate the EPR signal by lowering the temperature or increasing the microwave power. The static magnetic field is then held constant at the resonant field position. Because they satisfy the resonance conditions and are power saturated, the two states involved in the EPR transition are equally populated. Nuclear transitions (absorption of rf energy) then change the relative populations of the spin states, and the two states associated with the EPR signal are no longer equally populated. This allows more microwave photons to be absorbed by the unpaired electrons, and thus changes the EPR signal intensity. Figure 2.6 follows from Spaeth et $a l^{47}$ and illustrates the population of states at each step of the ENDOR process. 


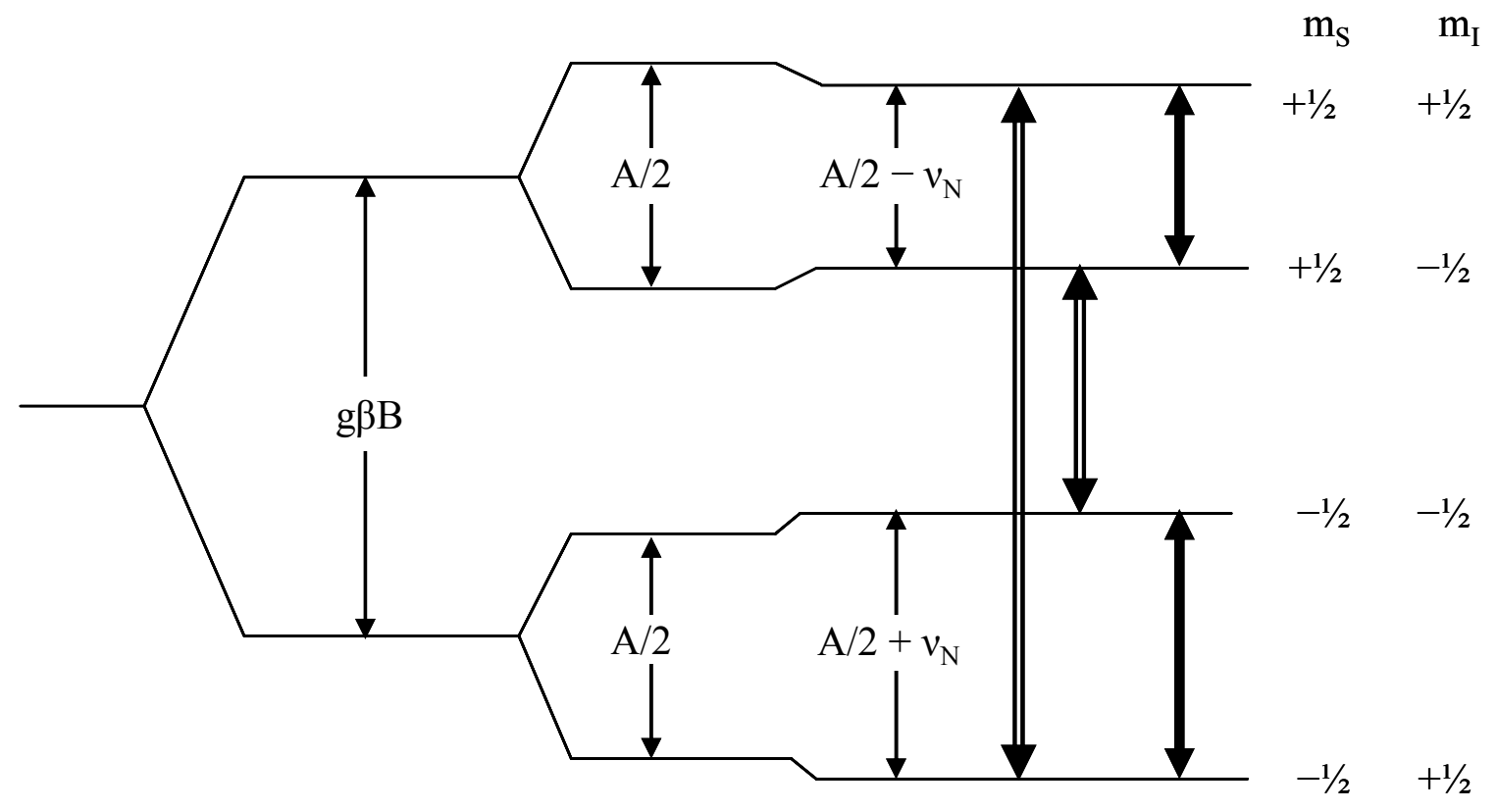

Figure 2.4. Energy level diagram for $\mathrm{A} / 2>v_{\mathrm{N}}$. Included are the electronZeeman, hyperfine, and nuclear-Zeeman effects. The double arrows represent EPR transitions, while the heavy solid arrows represent ENDOR transitions.

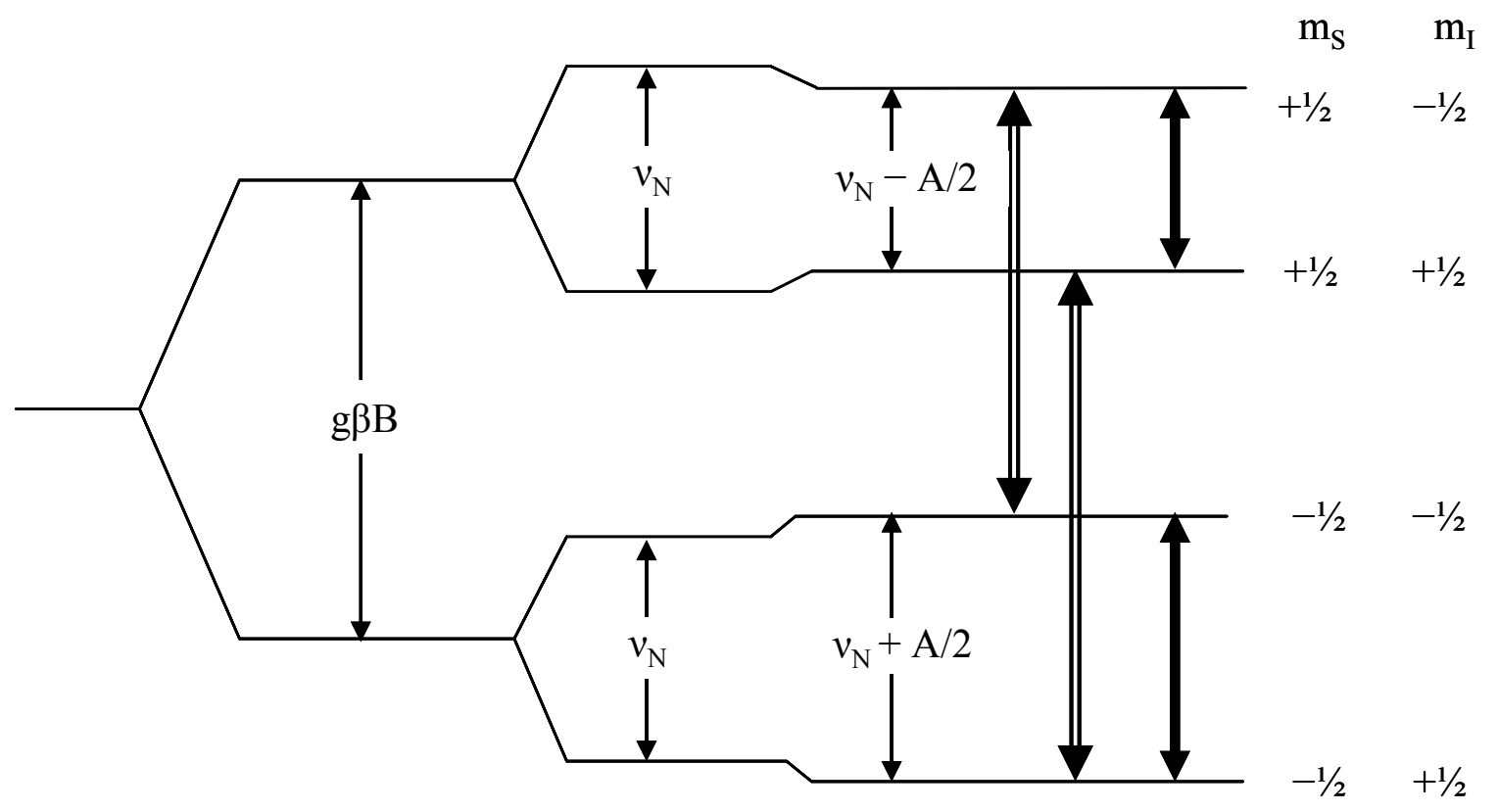

Figure 2.5. Energy level diagram for $v_{\mathrm{N}}>\mathrm{A} / 2$. Included are the electronZeeman, hyperfine, and nuclear-Zeeman effects. The double arrows represent EPR transitions, while the heavy solid arrows represent ENDOR transitions. 


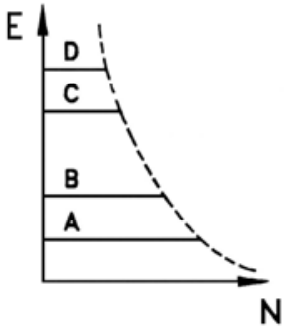

(a)

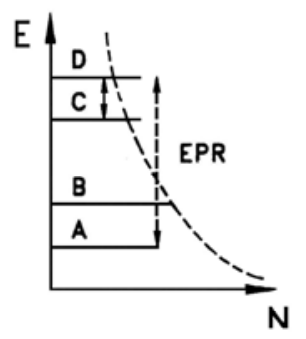

(c)

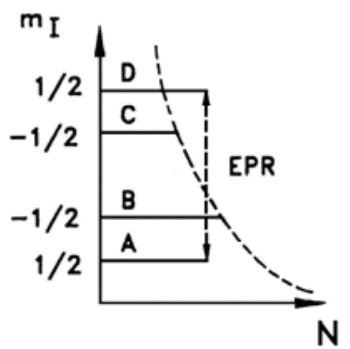

(b)

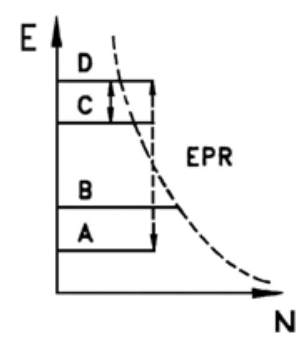

(d)

Figure 2.6. Diagrams of the population of spin states during different stages of EPR and ENDOR. (a) The states are initially in thermal equilibrium. (b) The population of states under a saturated EPR transition between states $A$ and $D$. (c) The states after an induced nuclear transition between $C$ and D. (d) The population of states after the EPR signal reaches a new equilibrium. (after Spaeth $^{47}$ )

\subsection{EPR Instrumentation}

The EPR spectra shown in this dissertation were taken on the X-band EMX Bruker spectrometer in Professor Giles' laboratory at West Virginia University. The system is commercially available from Bruker. The system has four main components: the microwave bridge, microwave cavity, electromagnet, and a computer-controlled acquisition system. Figure 2.7 is a general schematic of this EPR spectrometer. ${ }^{48}$

Most of the electronics pertaining to the microwaves are contained in the microwave bridge. The microwave source (1) is a Gunn diode (many older spectrometers used a klystron). Due to the constraints of fixed cavity dimensions (and thus a fixed cavity 


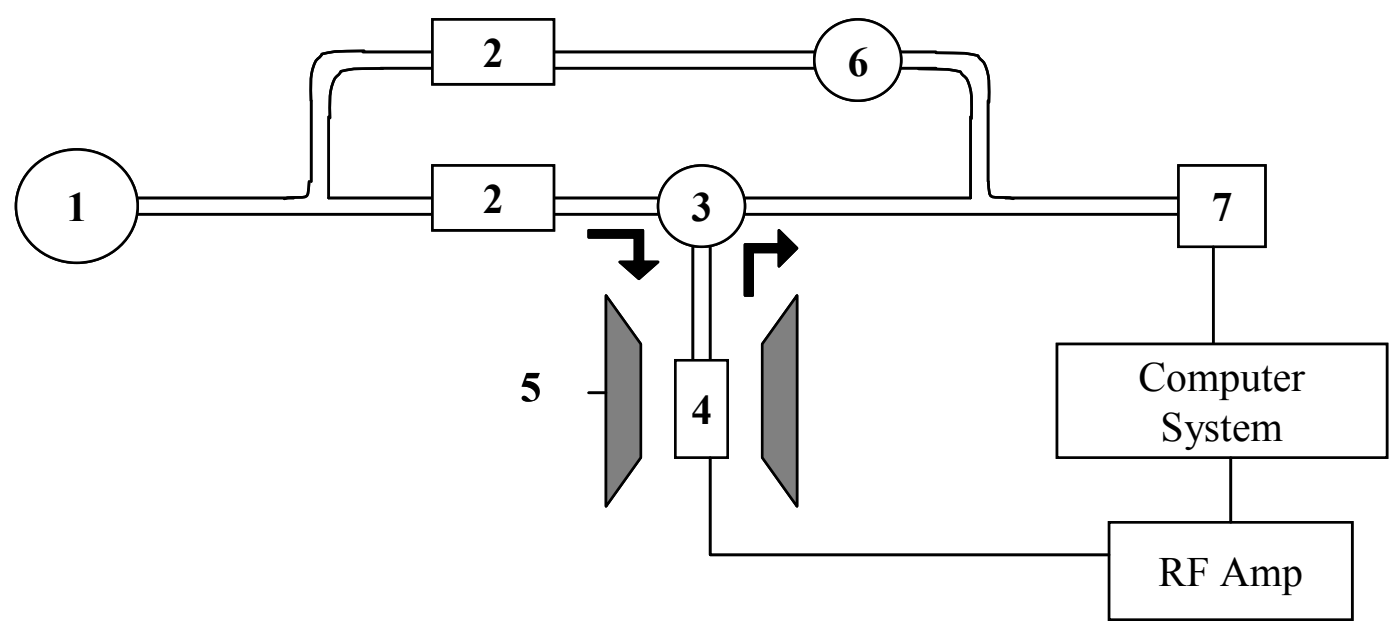

Figure 2.7. Diagram of a typical EPR system. Components 1, 2, 3, 6, and 7 all reside in the microwave bridge.

resonance frequency), the EPR spectrometer varies the magnetic field when taking spectra. This is done by varying the current through the electromagnet (5). In order to vary the power, the microwaves pass through two attenuators (2), one in the sample arm and one in the reference arm (i.e., a source of bias for the detector). The attenuator in the sample arm leads to a circulator (3), a ferrite device that controls the direction of flow of microwaves. The port from which microwaves emerge depends on the port the microwaves enter. Microwaves coming from the attenuator flow down to the microwave cavity, while microwaves coming from the cavity flow on towards a Schottky barrier diode detector (7). The circulator prevents microwaves reflected by the cavity from traveling back towards the Gunn diode. Since the microwaves incident on the detector are reflected from the cavity and no intermediate frequencies are generated, this type of spectrometer is referred to as a homodyne system. The detector is most sensitive when operating in the square-law regime (the diode current is proportional to the square root of the microwave power). A diode current of $200 \mathrm{~mA}$ is maintained using the attenuator in the sample arm and the attenuator and phase shifter in the reference arm. This phase 
shifter (6) is used to match the phase of the microwaves from the reference arm to the phase of the microwaves from the cavity as they converge on the diode detector.

The microwave cavity plays an extremely important role in EPR. Microwave cavities are metal and come in cylindrical or rectangular form. The resonant frequency of the cavity depends on its dimensions and the desired mode. The Gunn diode's output frequency is tuned to the resonant frequency of the cavity. The amount of microwave energy that is stored in the cavity is proportional to the $\mathrm{Q}$ factor of the cavity. The $\mathrm{Q}$ factor is defined as

$$
Q=\frac{2 \pi \text { (microwave energy stored in the cavity) }}{\text { energy dissipated per cycle }} .
$$

A practical "working" definition of the $\mathrm{Q}$ factor is

$$
Q=\frac{v_{\mathrm{r}}}{\Delta v}
$$

where $v_{\mathrm{r}}$ is the resonant frequency of the cavity and $\Delta v$ is the full width at half maximum of the resonance peak. Cylindrical cavities commonly have Q values of 5000 and rectangular cavities are typically 2000 to 3000 . Any material placed in the cavity that absorbs microwaves will lower the Q, and thus lower the sensitivity of the EPR spectrometer. Metal and water are obvious materials that will absorb microwaves, as well as dielectric sample holders made from Teflon or Delrin. Purging the microwave cavity with nitrogen gas is very important during low temperature experiments since water will condense on the outside of the cryogenic glassware placed inside the cavity.

Sample placement in the microwave cavity is important. Electric and magnetic standing waves are produced inside the cavity (the magnetic field maximum occurs at the electric field minimum). Since it is the magnetic field that induces the transitions, the highest sensitivity occurs when the sample is placed within the cavity at the magnetic field maximum (and the electric field minimum). There are two types of cavities used in the present work. The first is a $\mathrm{TE}_{102}$ mode rectangular cavity and is shown in Fig. 2.8, 


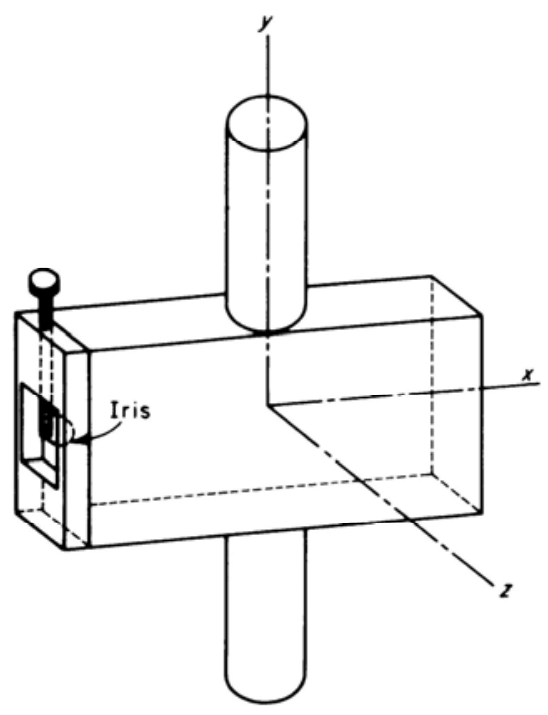

(a)

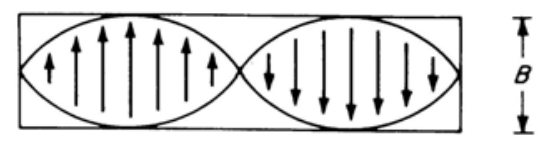

(b)

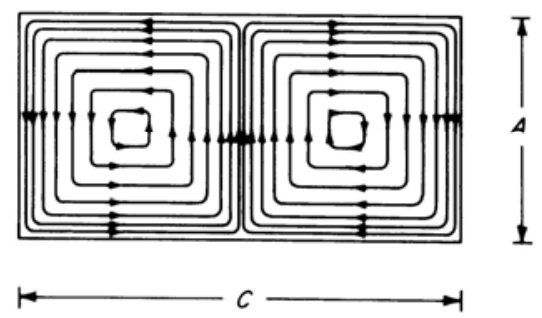

(c)

Figure 2.8. (a) Schematic of a $\mathrm{TE}_{102}$ rectangular cavity. (b) Electric field lines inside the cavity. (c) Magnetic field lines inside the cavity. (after Wertz and Bolton ${ }^{49}$ )

following Wertz and Bolton. ${ }^{49}$ Here, the middle of the cavity has a maximum magnetic field that is perpendicular to the static field from the electromagnet and so the sample is inserted into the cavity along this axis.

The second type of cavity is a $\mathrm{TE}_{011}$ cylindrical cavity shown in Fig. 2.9, also following Wertz and Bolton. ${ }^{49}$ Here, the electric field lines go around the central axis of the cavity with the electric field minimum along the central axis. In the present work, the ENDOR data is taken with a cylinder cavity containing an $\mathrm{rf}$ coil used to produce the $\mathrm{rf}$ fields necessary for ENDOR. A cylindrical cavity is used with the helix coil placed symmetrically around the central axis of the cavity to avoid decreasing the $\mathrm{Q}$ factor.

The most important aspect of an EPR spectrometer is matching the microwave cavity to the waveguide, i.e., impedance matching. The waveguide descending to the microwave cavity (see Fig. 2.7) is treated as a simple transmission line, and the cavity acts as a termination for this transmission line. When the cavity is matched to the waveguide (i.e., when the impedance of the termination is matched to the transmission 


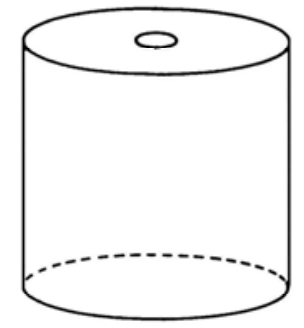

(a)

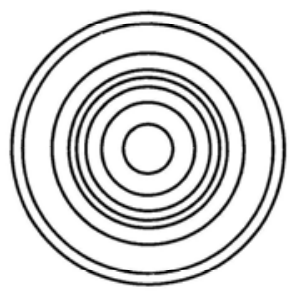

(b)

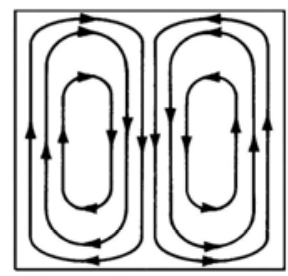

(c)

Figure 2.9. (a) Schematic of a $\mathrm{TE}_{011}$ cylindrical cavity. (b) Electric field lines inside the cavity. (c) Magnetic field lines inside the cavity.

line), there is no reflected microwave energy. In other words, all of the microwave energy is absorbed in the microwave cavity. During an EPR resonance, the impedance becomes slightly mismatched and microwave energy is reflected back up the waveguide to the circulator and ultimately to the detector diode. This is recorded as an EPR signal.

Through the years, methods have been developed to increase the signal-to-noise ratio of EPR spectrometers. A common method, introduced in the early years, was the use of phase-sensitive detection with a lock-in amplifier. Phase-sensitive detection requires that some aspect of the experiment be modulated. In EPR, it is the static magnetic field that is modulated. Small coils in the walls of the microwave cavity are driven at $100 \mathrm{kHz}$, thus producing a time-varying component of magnetic field parallel to the static magnetic field. The $100 \mathrm{kHz}$ oscillation of the static magnetic field modulates the intensity of the reflected microwaves as the static magnetic field is swept through resonance. The intensity of the noise produced in the detector diode is inversely proportional to the frequency used. A higher modulation frequency results in less noise. However, the $100 \mathrm{kHz}$ magnetic field must penetrate the thin metal walls of the cavity in order to reach the sample, and higher frequencies have less penetration into the cavity. The modulation frequency is chosen to balance noise and penetration; a $100 \mathrm{kHz}$ modulation frequency is an excellent compromise for EPR spectrometers. 

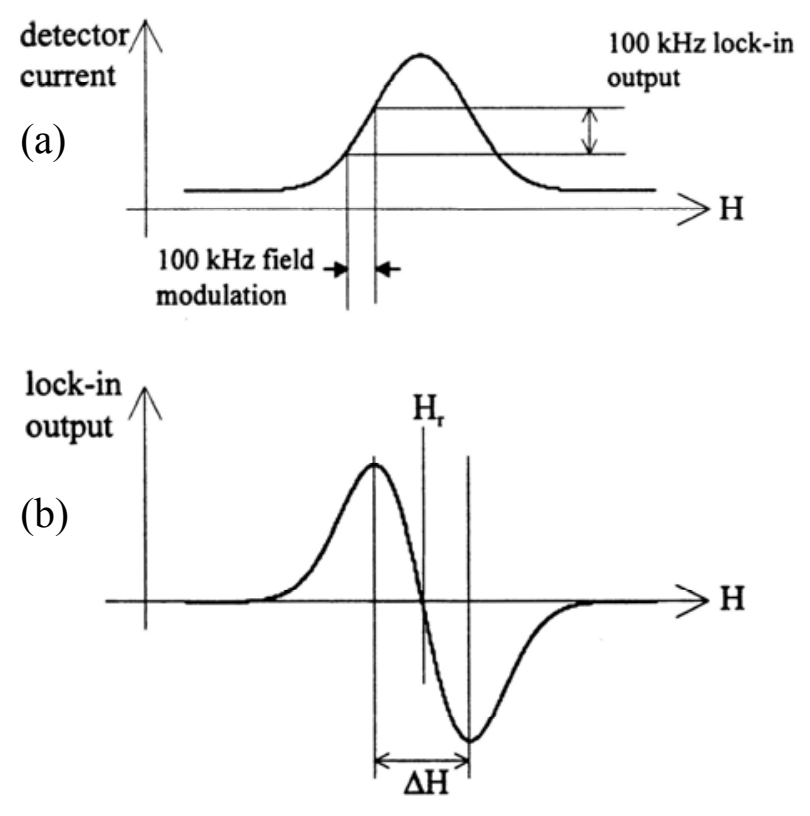

Figure 2.10. (a) Illustration of the effect of the field modulation on the amplitude of the microwave signal. (b) First-derivative output signal of the lock-in amplifier. (after Setzler ${ }^{48}$ )

Figure 2.10, following Setzler, ${ }^{48}$ illustrates the effect the field modulation has on the microwave output when the static field is near a resonance peak. The intensity of the modulated output signal is proportional to the slope of the absorption curve. For this reason the modulation amplitude must be smaller than the linewidth of the EPR curve. The lock-in amplifier filters out any signal not at $100 \mathrm{kHz}$ (or another specified modulation frequency). The phase-sensitive detection compares the phase of the signal to a reference. If the slope is positive, the signals are in phase and the result is a positive value. If the field is at the resonance peak, the slope is zero and thus the result is zero. If the slope is negative, the result is a negative value. This produces an output signal that is a first derivative of the absorption curve.

Many EPR experiments require controlled cryogenic temperatures. Helium-gasflow cryostats from Oxford Instruments are used. These crysostats contain glassware 
with vacuum hookups and are mounted and supported by the base of the cavity. The glassware has a tube that enters into the cavity and surrounds the sample holder. An evacuated transfer line provides a continuous flow of helium to the sample. Temperatures as low as 5 to $8 \mathrm{~K}$ can be achieved using this system.

\subsection{ENDOR Instrumentation}

ENDOR spectrometers are usually an extension of an EPR spectrometer. They utilize all of the components of the EPR spectrometer, with the addition of an rf source, an amplifier, and a coil within the cavity. The spectrometer used to obtain the data presented in this dissertation was a Bruker ELEXSYS EPR system with ENDOR capability. This spectrometer can be used for both EPR and ENDOR. The most important change is the placement of the aforementioned rf coil inside the microwave cavity. Since ENDOR experiments require cryogenic temperatures, the rf coil is simply mounted on the glassware that runs through the cavity, as discussed below. The static magnetic field is held constant on an EPR resonance, while the frequency of the oscillator feeding the rf coil is varied. The rf generator in this spectrometer is capable of producing output from 0.5 to $200 \mathrm{MHz}$. The ouput of the oscillator is fed through an amplifier having a frequency range extending to $105 \mathrm{MHz}$. The static magnetic field is not modulated during an ENDOR experiment, instead the rf is frequency modulated. The same principles apply for the phase-sensitive detection, and a first-derivative shape results. 


\section{CHAPTER 3}

\section{Oxygen Donors in Aluminum Nitride}

\subsection{Introduction}

\subsubsection{Crystal Structure}

Aluminum nitride (AIN) is a III-V compound semiconductor with the wurtzite crystal structure belonging to the $\mathrm{P} 6_{3} \mathrm{mc}$ space group. ${ }^{50}$ The wurtzite structure is similar to the zinc-blende structure. The zinc-blende structure is cubic with the four neighbors forming a perfect tetrahedron about each lattice site (i.e., the four neighbors are at the corners of a cube). In contrast, the wurtzite structure of AlN is hexagonal with the four neighbors surrounding a lattice site forming a distorted tetrahedra (three equivalent neighbors lie in the basal plane while the fourth neighbor with a different bond length defines the $c$ axis). The local structure of the AlN lattice is shown in Fig. 3.1. Each aluminum atom is bonded to four nitrogen atoms, thus forming the distorted tetrahedron around the aluminum atom. Similarly, the four neighboring aluminum atoms bonded to a nitrogen atom form an equivalent distorted tetrahedron.

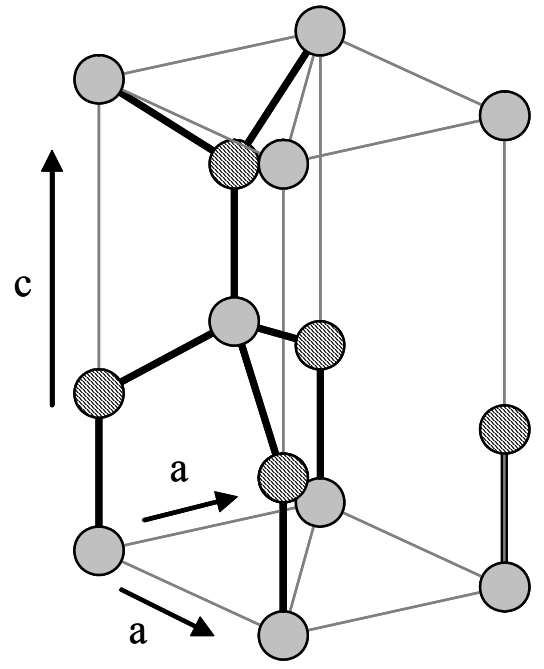

(a)

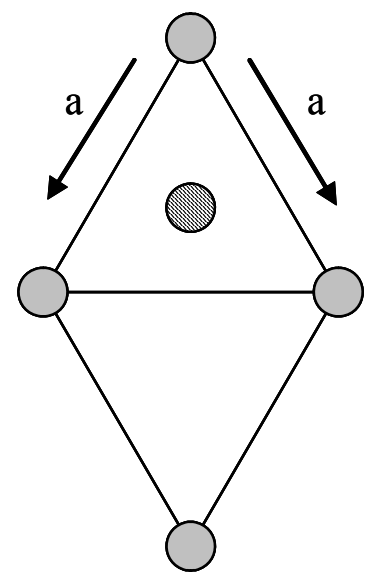

Aluminum atom

Nitrogen atom

Figure 3.1. Unit cell of aluminum nitride. (a) Three-dimensional view. (b) Projection on the basal plane. 
The lattice parameters for AlN are $a=3.11 \AA$ and $c=4.98 \AA .^{50,51}$ This crystal structure is defined by a four-atom basis at each point of the hexagonal lattice. Starting with a cation at the origin, the positions of the three remaining atoms in the basis are given in Table 3.1 as a fraction of the lattice constant in the corresponding direction.

\begin{tabular}{|c|c|c|}
\hline Atom & Position (a,a,c) & Position (a,a,c) \\
\hline Cation & $(0,0,0)$ & $(0.5,0.5,0.5)$ \\
\hline Anion & $(0,0, u)$ & $(0.5,0.5,0.5+u)$ \\
\hline
\end{tabular}

Table 3.1. Positions of basis atoms in a wurtzite unit cell.

The parameter $u$ in Table 3.1, when multiplied by $c$, gives the length of the Al-N bond parallel to the $c$ axis. For an ideal wurtzite structure, the value of the parameter $u$ is $3 / 8$ (or 0.375$).{ }^{52}$ In the ideal wurtzite structure, the four nearest neighbors of an atom form a perfect tetrahedron about that atom. Aluminum nitride deviates slightly from the ideal wurtzite structure and has a value of 0.385 for the parameter $u{ }^{53}$ The ratio c/a is 1.600 for AlN, while c/a is 1.633 in the ideal wurtzite structure. ${ }^{52}$ For AlN, the positions of the four basis atoms are listed in Table 3.2. These crystallographic results for AIN translate into bond lengths of $1.917 \AA$ for the axial neighbor and $1.885 \AA$ for the three basal plane neighbors.

\begin{tabular}{|c|c|c|}
\hline Atom & Position (a,a,c) & Position (a,a,c) \\
\hline $\mathrm{Al}$ & $(0,0,0)$ & $(0.5,0.5,0.5)$ \\
\hline $\mathrm{N}$ & $(0,0,0.385)$ & $(0.5,0.5,0.885)$ \\
\hline
\end{tabular}

Table 3.2. Positions of basis atoms in a unit cell of AlN. 


\subsubsection{Crystallographically Equivalent Defect Orientations}

The symmetry operations of a crystal must often be considered when analyzing and interpreting experimental results. These symmetry operations are especially important in magnetic resonance experiments, as the EPR and ENDOR spectra may shift in field position and split into components as the magnetic field is rotated relative to the crystal axis. The splittings that occur when the magnetic field is rotated away from a high-symmetry direction are due to multiple orientations of the same defect within the crystal. These multiple orientations are referred to as crystallographically equivalent sites of the defect (they become magnetically inequivalent when the field is along an arbitrary direction). The different sites corresponding to a particular defect are related by symmetry operations.

Aluminum nitride, and the wurtzite structure in general, belong to the $\mathrm{P}_{3} \mathrm{mc}$ space group. Rotations of $120^{\circ}$ (i.e., $120^{\circ}, 240^{\circ}, 360^{\circ}$ ) about the $c$ axis reproduce the original lattice. Rotations of $60^{\circ}, 180^{\circ}$, and $300^{\circ}$ (i.e., odd multiples of $60^{\circ}$ ) about the $c$ axis reproduce the original lattice when followed by a reflection through the $c-a$ plane or a set of translations along the $a$ and $c$ axes. Another important symmetry operation is a reflection through a plane perpendicular to the $a$ axis. The local symmetry of a defect, coupled with the symmetry of the crystal, must be known when predicting the angular behavior of EPR and ENDOR spectra. Important factors include the number of crystallographically equivalent but magnetically inequivalent defect sites expected for a single orientation of magnetic field and the expected orientation of the interaction matrices of the spin Hamiltonian. Having information about the local geometries at the defect site leads to a better understanding of observed angular behavior of the EPR and ENDOR spectra when the magnetic field is rotated relative to the crystal axes. In the absence of hyperfine splitting, an $S=1 / 2$ defect will have the same number of signals as there are sites. Degeneracies in the EPR spectra (when two or more EPR signals occur at the same value of magnetic field) often occur for certain high-symmetry orientations of 
magnetic field. This information concerning symmetries is significant in identifying defects and in determining the local distribution of the unpaired spin.

When analyzing the spin Hamiltonian for a hexagonal crystal system, special care must be exercised when defining the coordinate system in which the spin Hamiltonian is written. As shown in Chapter 2, for a system with $S=1 / 2$ and $I>1 / 2$, the spin Hamiltonian can be written as

$$
\hat{H}=\beta \mathbf{B} \cdot \overrightarrow{\mathbf{g}} \cdot \hat{\mathbf{S}}+\hat{\mathbf{I}} \cdot \overrightarrow{\mathbf{A}} \cdot \hat{\mathbf{S}}+\hat{\mathbf{I}} \cdot \overrightarrow{\mathbf{Q}} \cdot \hat{\mathbf{I}}-\mathrm{g}_{\mathrm{N}} \beta_{\mathrm{N}} \mathbf{B} \cdot \hat{\mathbf{I}}
$$

where $\overrightarrow{\mathbf{g}}, \overrightarrow{\mathbf{A}}$, and $\overrightarrow{\mathbf{Q}}$ are three-by-three matrices. For example, each matrix can be written as

$$
\vec{X}=\left(\begin{array}{lll}
X_{11} & X_{12} & X_{13} \\
X_{21} & X_{22} & X_{23} \\
X_{31} & X_{32} & X_{33}
\end{array}\right)
$$

where the subscripts 1,2 , and 3 correspond to the three orthogonal directions of the coordinate system in which the matrix is being expressed. In order to extract energy eigenvalues that can be compared to experiment, all the terms in the spin Hamiltonian need to be written in a common coordinate system. This is often done by first writing each of the matrices in their respective principal axis systems (the systems in which each matrix is diagonal). Then, rotation matrices (defined in terms of Euler angles) transform the principal-axis coordinate system to the crystal coordinate system). This procedure leads to a spin Hamiltonian expressed in a single coordinate system.

For a hexagonal crystal such as AlN, care must be taken in defining the directions of the principal axes of each spin Hamiltonian matrix relative to the crystal axes. This task is easier for orthorhombic, tetragonal or cubic crystal structures. The four high symmetry directions for AlN do not define an orthogonal crystal coordinate system. One of these four is the $c$ axis, which is defined to be along the unique direction of the crystal lattice (see Fig. 3.1). The remaining three high-symmetry directions lie in the plane perpendicular to the $c$ axis and are $120^{\circ}$ apart as shown in Fig. 3.2. 


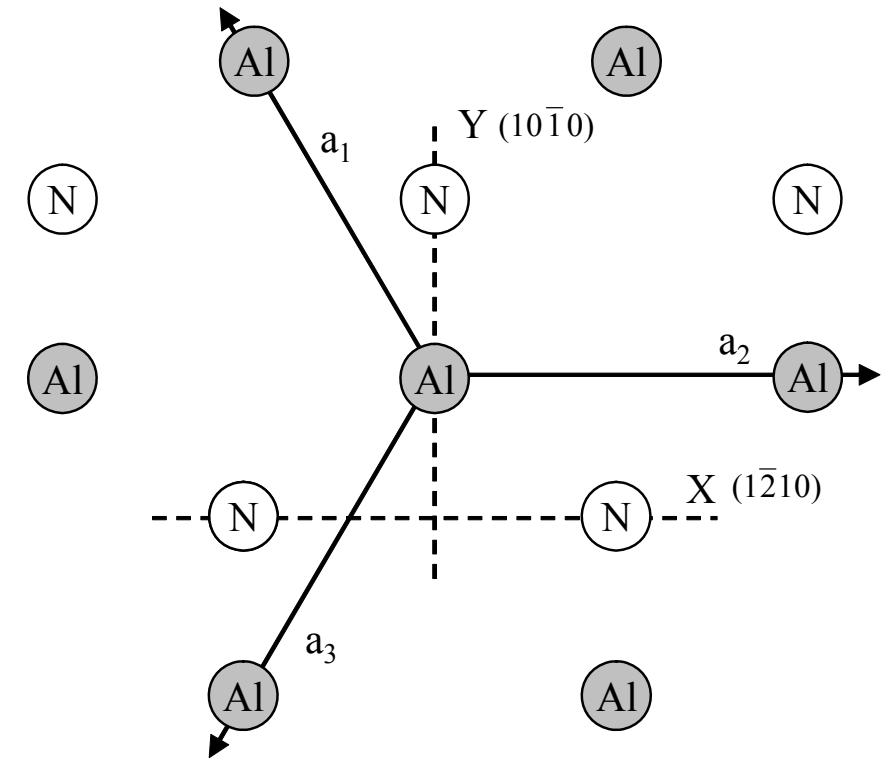

Figure 3.2. Projection of the AIN crystal structure on the basal plane.

Solid arrows indicate the crystal high-symmetry directions $\left(\mathrm{a}_{1}, \mathrm{a}_{2}, \mathrm{a}_{3}\right)$, while the dashed lines $(\mathrm{X}, \mathrm{Y})$ indicate the orthogonal crystal coordinate system.

Since the spin-Hamiltonian matrices must be written in an orthogonal coordinate system, an appropriate coordinate system must be defined specifically for this task. This coordinate system must be used when rewriting each matrix in the spin Hamiltonian. The common coordinate system of the spin Hamiltonian and the principal-axes coordinate systems of the matrices are related by a set of three Euler angles for each respective matrix. These Euler angles define the rotation matrices that rotate the principal-axis system into the common coordinate system. An example of a common coordinate system is shown in Fig. 3.2. The coordinate system $\mathrm{X}, \mathrm{Y}, \mathrm{Z}$ is the common crystal coordinate system. The $\mathrm{Z}$ axis is defined to be along the $c$ axis of the crystal, the $\mathrm{X}$ axis is defined to be along one of the three high-symmetry directions, $a$, and the $\mathrm{Y}$ axis is chosen to complete an orthogonal coordinate system (note that $\mathrm{Y}$ is not a high-symmetry direction in the crystal). There are three equivalent ways to define the $\mathrm{X}$ direction, i.e., there are three $a$ directions in the basal plane separated by $120^{\circ}$ rotations about the $c$ axis. In 
general, a defect will have a minimum of six distinguishable orientations in the AlN lattice. Miller indices (ijkl) are used to describe specific directions in wurtzite crystals. The index 1 refers to the $c$ axis, and the indices $\mathrm{i}, \mathrm{j}, \mathrm{k}$ refer to the three $a$ axes $\left(\mathrm{a}_{1}, \mathrm{a}_{2}, \mathrm{a}_{3}\right)$. When specifying directions, the usual convention is followed where $k=-(i+j)$. For the system defined in Fig. 3.2, the directions are defined as $(1 \overline{2} 10)$ for the $\mathrm{X}$ axis and (1010) for the $\mathrm{Y}$ axis using Miller indices.

\subsubsection{Previous Defect Studies in Aluminum Nitride}

Single crystals of AlN are excellent candidates for use as substrates in the epitaxial production of III-nitride electronic and optoelectronic devices. ${ }^{1}$ Although significant progress has been made in the growth of bulk AlN crystals, ${ }^{54-58}$ the investigation of impurities and native defects in this material is still in a preliminary stage. It is widely acknowledged that oxygen is the primary unintentional impurity in AlN crystals ${ }^{4-8}$ and that native defects, such as nitrogen vacancies and aluminum vacancies, also exist. ${ }^{9-12}$ These oxygen impurities and native defects are present at significant concentrations in many AlN crystals, and can greatly influence the optical and electrical properties of the crystals. To maintain progress in the development of high quality AlN substrates, it is important to use optical absorption, luminescence, and magnetic resonance to identify "characteristic" spectra for each of the major donors and acceptors. Results from these experiments will also complement the first-principles calculations that are being used to investigate the structure and energetics of point defects in the III-nitrides. ${ }^{59}$

Electron paramagnetic resonance (EPR), electron nuclear double resonance (ENDOR), and optically detected magnetic resonance have proven to be effective techniques for identifying point defects in semiconductors. Only a few magnetic resonance studies, however, have been reported for AIN. Atobe et al. ${ }^{60}$ and Honda et $a l .{ }^{50}$ attributed an EPR signal near $\mathrm{g}=2.007$ in neutron-irradiated AlN ceramics to the 
nitrogen vacancy. In a separate investigation of AlN ceramics, Schweizer et al. ${ }^{51}$ suggested that an EPR signal near $\mathrm{g}=2.004$ and its associated ENDOR was due to an oxygen on a nitrogen site with an adjacent aluminum vacancy and that a photoluminescence-EPR signal near $\mathrm{g}=1.990$ was due to an isolated oxygen substituting for nitrogen. The ceramic nature of these samples ${ }^{50,51,60}$ did not allow the angular dependence of the spectra to be investigated. In contrast, Mason et al. ${ }^{61}$ used optically detected EPR (OD-EPR) to observe a series of $S=1$ and $S=1 / 2$ spectra in single crystals of AlN where angular dependence data were available. They suggested that one of their $\mathrm{S}=1 / 2$ spectra (labeled D5, with $\mathrm{g}_{\|}=2.001$ and $\mathrm{g}_{\perp}=2.007$ ) was associated with a displaced host aluminum atom.

In this chapter, the results of an EPR and ENDOR study of single crystals of AIN are described. A broad EPR signal near $\mathrm{g}=2.0$ is present in the as-grown crystals, and its intensity becomes much larger when a sample is irradiated at room temperature with $\mathrm{x}$ rays. The ENDOR results show that this defect has a large hyperfine interaction with one ${ }^{27} \mathrm{Al}$ nucleus ( $\mathrm{I}=5 / 2,100 \%$ abundant) and weaker, but equivalent, hyperfine interactions with three other aluminum nuclei. The axial symmetry of this center and the hyperfine interactions with neighboring aluminums suggest that the responsible defect is an isolated donor on a nitrogen site, either a neutral oxygen substituting for nitrogen $\left(\mathrm{O}_{\mathrm{N}}^{0}\right)$ or a neutral nitrogen vacancy $\left(\mathrm{V}_{\mathrm{N}}^{0}\right)$. This EPR spectrum appears to be the same as the D5 spectrum reported by Mason et al. ${ }^{61}$ but a different assignment is proposed here. Mason et al.$^{61}$ observed an increase in their D5 spectrum after irradiating with high-energy electrons, and suggested that an atomic displacement may have occurred. A similar increase in this signal is observed during an irradiation with $\mathrm{x}$ rays, but only pre-existing defects are considered as possible models for this center since the $\mathrm{x}$ rays are not expected to produce displacements in this material. The $\mathrm{x}$-irradiation produces transient changes in charge states, lasting for hours or days at room temperature, that result from the capture of radiation-induced electrons and holes. 


\subsection{Experimental Results}

\subsubsection{EPR Study}

The four bulk AlN crystals used in the present investigation were grown by a selfseeded sublimation-recondensation technique at Crystal IS (Green Island, NY). These small c-plate samples with typical dimensions of $5.0 \times 3.0 \times 1.3 \mathrm{~mm}^{3}$ were cut from a larger boule. The EPR and ENDOR data were taken near $9.48 \mathrm{GHz}$ using Bruker EMX and ELEXSYS spectrometers, respectively, while Oxford Instruments ESR-900 heliumgas-flow systems maintained the temperature of the samples at selected values in the 20 to $40 \mathrm{~K}$ range. Values of the static magnetic field were obtained with a proton gaussmeter. A small $\mathrm{Cr}$-doped $\mathrm{MgO}$ crystal was used to correct for the difference in magnetic field between the sample and the probe tip of the gaussmeter (the isotropic $\mathrm{g}$ value for $\mathrm{Cr}^{3+}$ in $\mathrm{MgO}$ is 1.9800). The source of $\mathrm{x}$ rays was a Varian OEG-76H tube operating at $60 \mathrm{kV}$ and $30 \mathrm{~mA}$.

The as-grown AlN crystals had a slight reddish color due to the presence of one or more weak defect-related optical absorption bands peaking in the 350 to $450 \mathrm{~nm}$ region. A small, but easily detected, EPR signal was also present in the as-grown crystals. Exposure to ionizing radiation (i.e., $\mathrm{x}$ rays) at $77 \mathrm{~K}$ or room temperature for $30 \mathrm{~min}$ caused the EPR signal to increase by a factor between 5 and 10 (depending on the sample) and also caused the short-wavelength visible absorption to increase (making the sample appear more red to the eye). This effect was stable at room temperature as the EPR signal only returned to its original intensity after several days if the sample was kept in the dark at room temperature. However, this restoration occurred in hours at both room temperature and in liquid nitrogen if the sample was held in room light. Figure 3.3 shows the EPR spectra taken at $40 \mathrm{~K}$ with the magnetic field parallel and perpendicular to the crystal's $c$ axis. These signals in Fig. 3.3 correspond to a concentration of spins of approximately $7 \times 10^{18} \mathrm{~cm}^{-3}$. There is a slight shift of the center of the EPR spectrum when the magnetic field is rotated from the $c$ axis to the basal plane, and no shift when 
(a)
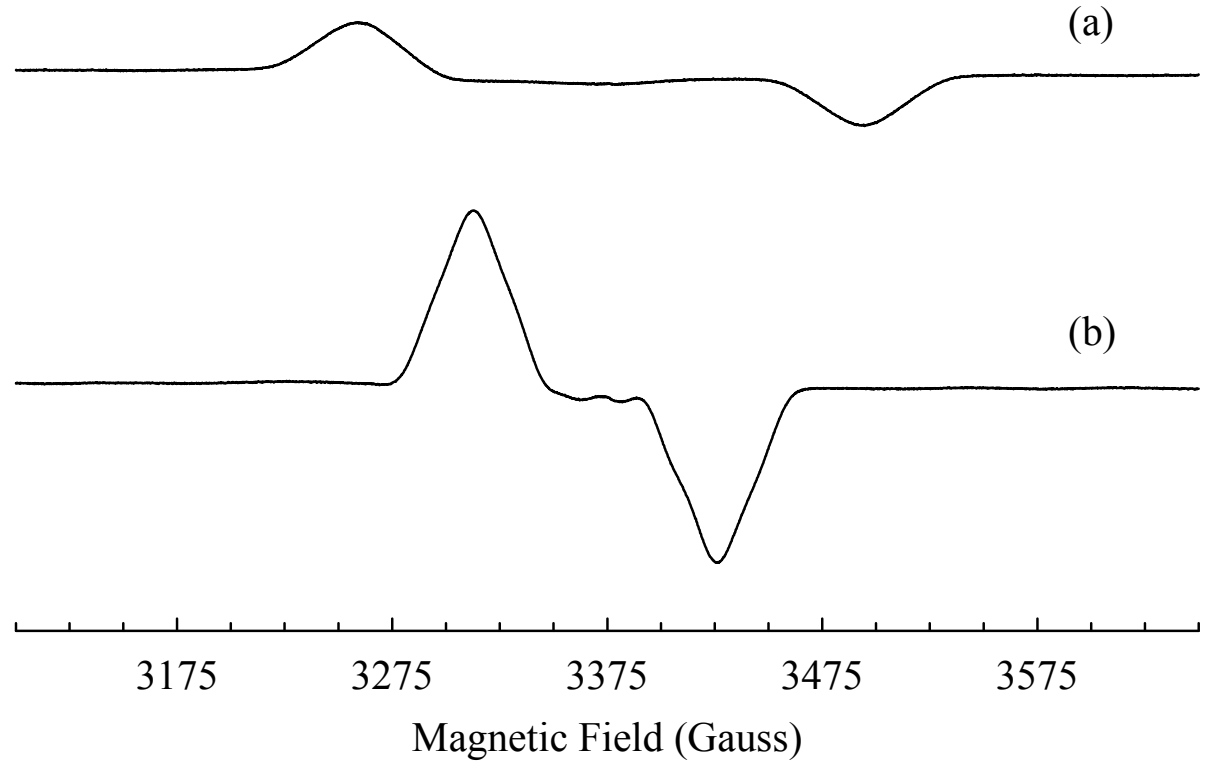

Figure 3.3. EPR from a donor in a single crystal of AlN. The sample was irradiated with $\mathrm{x}$ rays at room temperature to increase the intensity of the EPR signal. These data were taken at $40 \mathrm{~K}$ with the magnetic field (a) parallel to the $c$ axis and (b) perpendicular to the $c$ axis. The microwave frequency was $9.480 \mathrm{GHz}$.
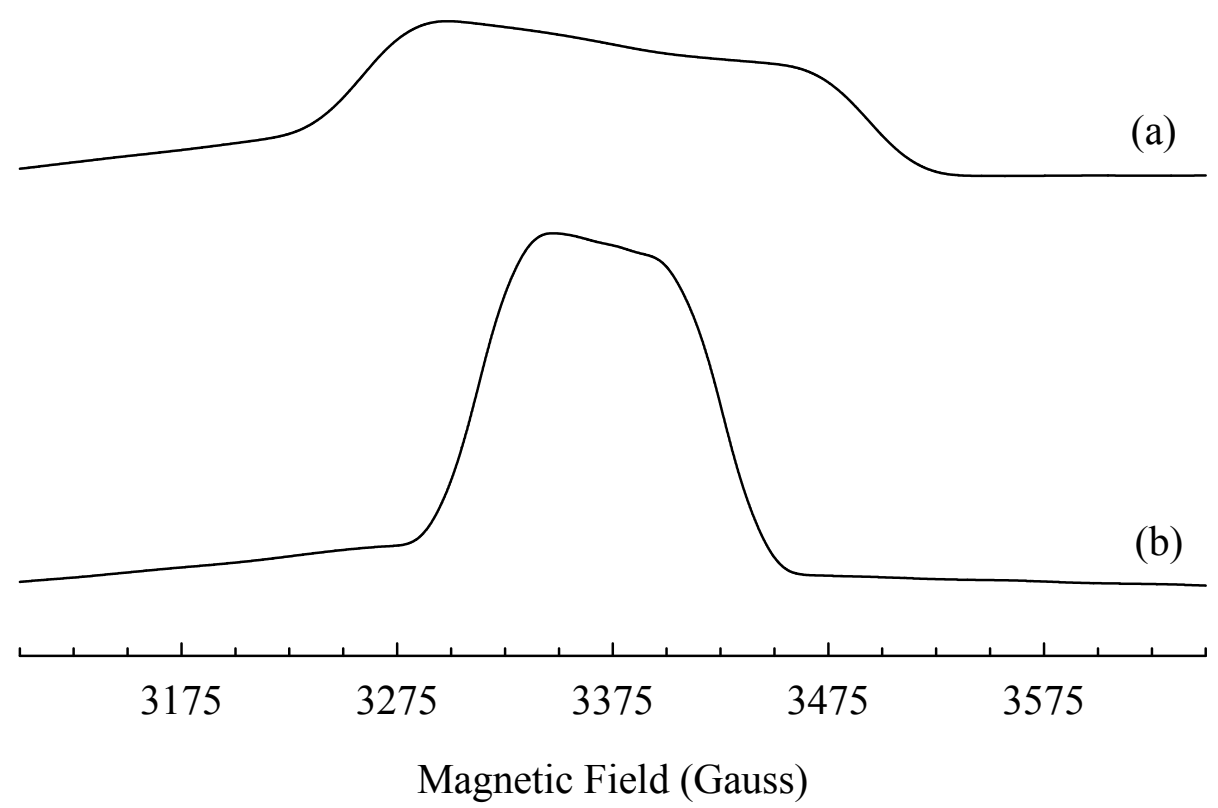

Figure 3.4. Integration of the spectra in Fig. 3.3. This shows the top-hat shape of the absorption curve for the magnetic field (a) parallel to the $c$ axis and (b) perpendicular to the $c$ axis. 
the magnetic field is rotated within the basal plane. This indicates an axial g matrix. By choosing the centers of the two spectra in Fig. 3.3 to be midway between the upper and lower peaks on each side, the $g$ values were found to be $g_{\|}=2.002$ and $g_{\perp}=2.006$.

The most noticeable feature of this EPR spectrum is the shape of the signal. The EPR signal does not have the usual derivative shape. The absorption curve represented by the EPR signal has a "top hat" shape which makes the first derivative shown in Fig. 3.3 extend up at lower field, stay near zero for a center field region, and extend down at higher field. To illustrate the "top hat" shape of the absorption, the EPR spectra in Fig. 3.3 were integrated to produce the corresponding absorption curves and the results are shown in Fig. 3.4. This absorption shape can be explained by multiple bands that overlap in such a way to produce the observed plateau effect.

\subsubsection{ENDOR of the Axial ${ }^{27}$ Al Neighbor}

The EPR spectrum in Fig. 3.3 displays some interesting behavior and features. As noted before, there is the irregular line shape. Also, the width of the spectrum in Fig. 3.3 changes significantly as the magnetic field is rotated from the $c$ axis to the basal plane (approximately $238 \mathrm{G}$ when the field is parallel to the $c$ axis and $116 \mathrm{G}$ when the field is perpendicular to the $c$ axis). ENDOR can be used to study the unresolved hyperfine associated with this EPR signal. The ENDOR spectrum shown in Fig. 3.5 was taken with the magnetic field oriented perpendicular to the $c$ axis, and with the field set at a value corresponding to the middle of the EPR signal. The ENDOR spectrum for this orientation shows two sets of five lines. With resolved hyperfine, an ENDOR spectrum would contain two sets of at most two signals for each EPR line. For the EPR spectra in Fig. 3.3, the hyperfine splitting is unresolved. The EPR signals overlap, allowing all the ENDOR transition to be observed in Fig 3.5 for one magnetic field value. When the magnetic field is parallel to the $c$ axis, the EPR signals spread out far enough that not all the ENDOR signals can be observed by setting the magnetic field at just one value. 
The ENDOR spectrum in Fig. 3.5 shows that there is a large hyperfine interaction with one aluminum nucleus, as illustrated by the two sets of five lines centered at 23.31 and $31.05 \mathrm{MHz}$. These two sets are located at $\mathrm{A} / 2 \pm v_{\mathrm{N}}$. They are centered on $\mathrm{A} / 2$ and separated by $2 v_{\mathrm{N}}$. The observed $7.74 \mathrm{MHz}$ separation of these two sets agrees with the prediction of $7.51 \mathrm{MHz}$ for $2 v_{\mathrm{N}}$ for the "free" ${ }^{27} \mathrm{Al}$ nucleus at $3382 \mathrm{G}$. Additional evidence that the ${ }^{27} \mathrm{Al}$ nucleus is responsible for the ENDOR spectrum in Fig. 3.5 comes from the five lines in each set. These lines arise from a nuclear electric quadrupole interaction involving an $\mathrm{I}=5 / 2$ nucleus. To better explain how this information is helpful in assigning ${ }^{27} \mathrm{Al}$ as the responsible nucleus for the observed pattern, Fig. 3.6 shows the energy-level diagram for an $\mathrm{I}=5 / 2$ nucleus such as ${ }^{27} \mathrm{Al}$, and Table 3.3 gives energy values for these levels in terms of the hyperfine parameters. The EPR transitions $\left(\Delta \mathrm{m}_{\mathrm{S}}=\right.$ $\left.\pm 1, \Delta \mathrm{m}_{\mathrm{I}}=0\right)$ and the ENDOR transitions $\left(\Delta \mathrm{m}_{\mathrm{S}}=0, \Delta \mathrm{m}_{\mathrm{I}}= \pm 1\right)$ are shown in Fig. 3.6.

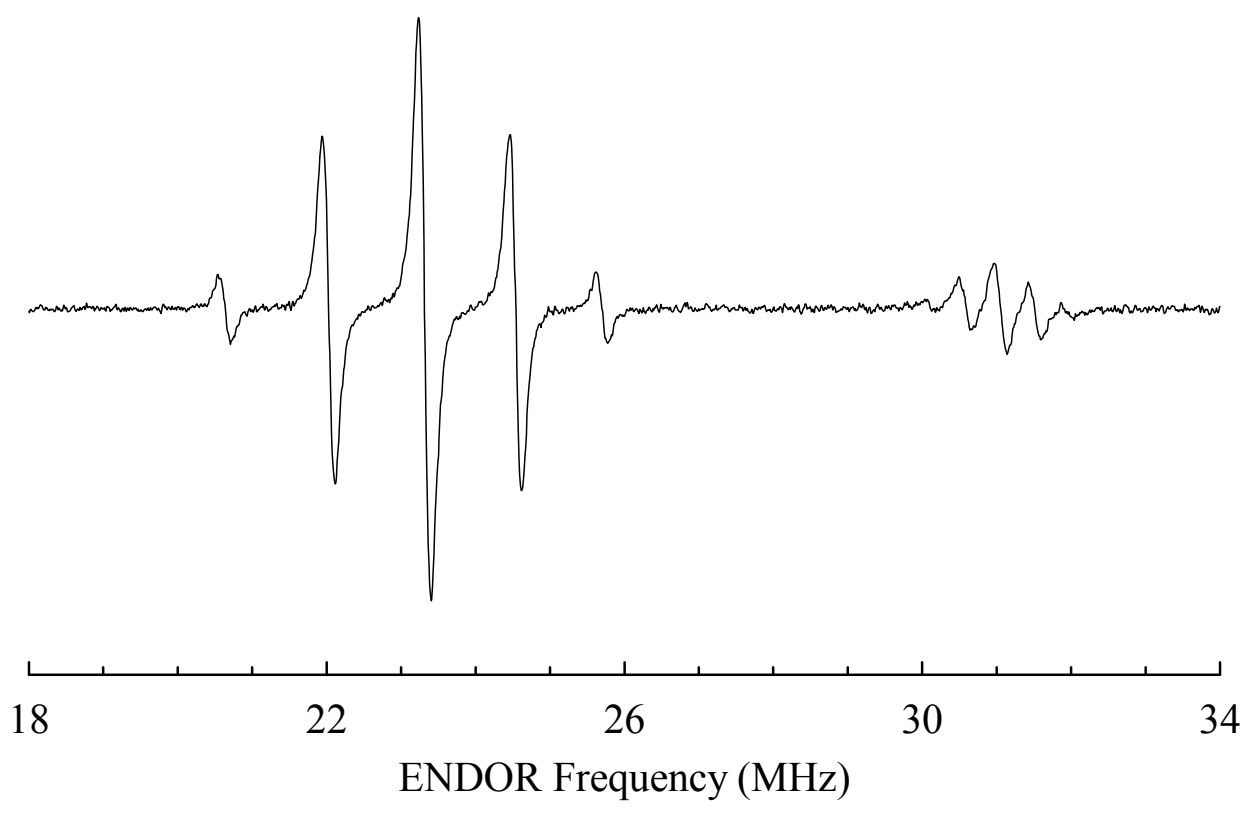

Figure 3.5. An ENDOR spectrum from AlN showing the primary aluminum hyperfine interaction. These data were taken at $22 \mathrm{~K}$ with the magnetic field $(3382 \mathrm{G})$ perpendicular to the $c$ axis. The five lines in each set result from the nuclear electric quadrupole interaction with the $\mathrm{I}=5 / 2$ ${ }^{27} \mathrm{Al}$ nucleus. 


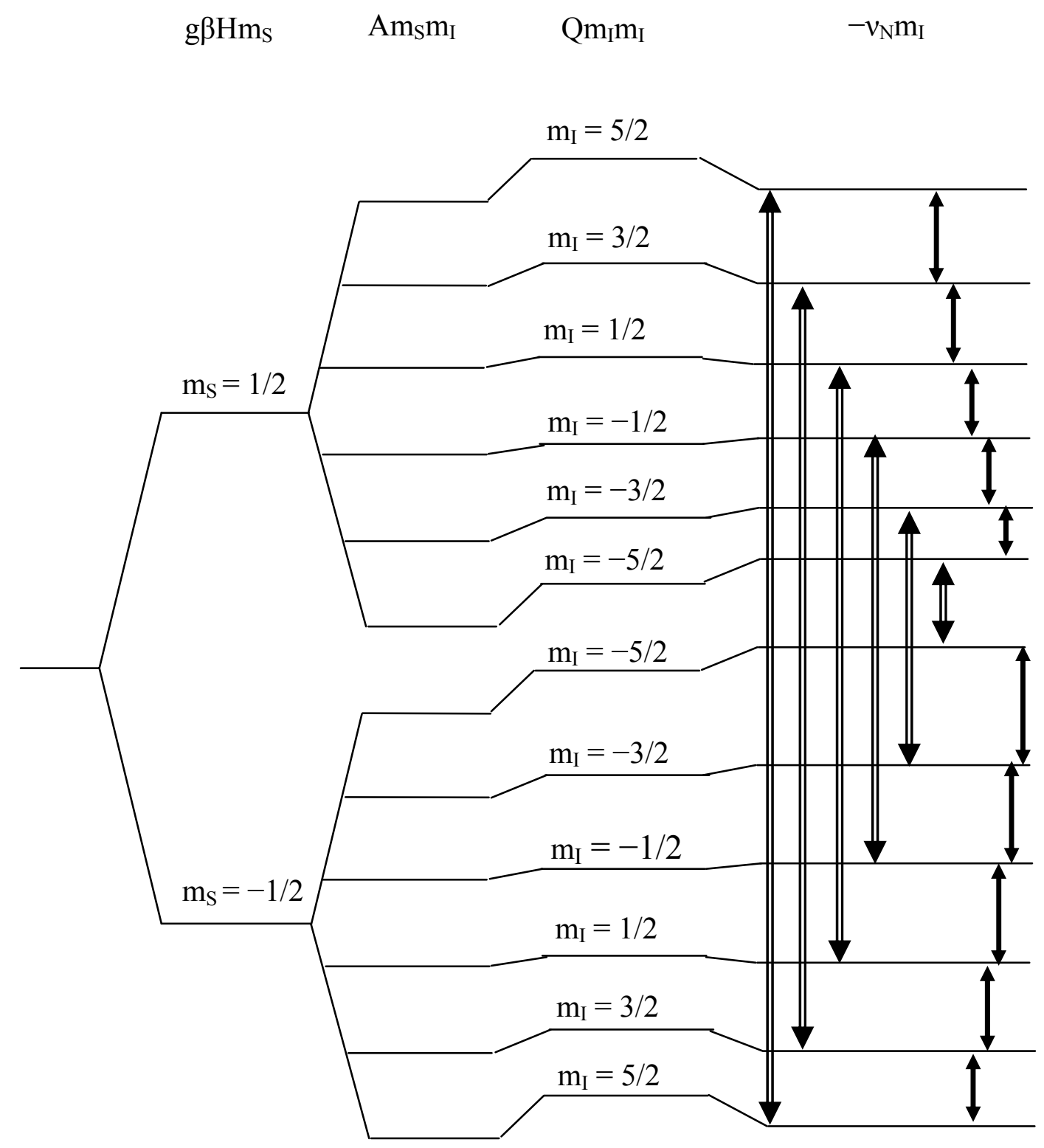

Figure 3.6. Energy level diagram of a spin system with $\mathrm{S}=1 / 2$ and $\mathrm{I}=$ $5 / 2$. The diagram shows the splitting caused by each term in the spin Hamiltonian. Each energy level is labeled by the appropriate $m_{I}$ value. EPR and ENDOR transitions are indicated by the double and single arrows, respectively. Here, the parameters $A, Q$, and $v_{N}$ are taken to be positive and $\mathrm{A} / 2$ is greater than $v_{\mathrm{N}}$. 


\begin{tabular}{|c|c|}
\hline$\left(\mathrm{m}_{\mathrm{S}}, \mathrm{m}_{\mathrm{I}}\right)$ & Energies of Spin Levels \\
\hline$(1 / 2,5 / 2)$ & $\frac{1}{2} \beta \mathrm{gH}+\frac{5}{4} \mathrm{~A}+\frac{25}{4} \mathrm{Q}-\frac{5}{2} v_{\mathrm{N}}$ \\
$(1 / 2,3 / 2)$ & $\frac{1}{2} \beta \mathrm{gH}+\frac{3}{4} \mathrm{~A}+\frac{9}{4} \mathrm{Q}-\frac{3}{2} v_{\mathrm{N}}$ \\
$(1 / 2,1 / 2)$ & $\frac{1}{2} \beta \mathrm{gH}+\frac{1}{4} \mathrm{~A}+\frac{1}{4} \mathrm{Q}-\frac{1}{2} v_{\mathrm{N}}$ \\
$(1 / 2,-1 / 2)$ & $\frac{1}{2} \beta \mathrm{gH}-\frac{1}{4} \mathrm{~A}+\frac{1}{4} \mathrm{Q}+\frac{1}{2} v_{\mathrm{N}}$ \\
$(1 / 2,-3 / 2)$ & $\frac{1}{2} \beta \mathrm{gH}-\frac{3}{4} \mathrm{~A}+\frac{9}{4} \mathrm{Q}+\frac{3}{2} v_{\mathrm{N}}$ \\
$(1 / 2,-5 / 2)$ & $\frac{1}{2} \beta \mathrm{gH}-\frac{5}{4} \mathrm{~A}+\frac{25}{4} \mathrm{Q}+\frac{5}{2} v_{\mathrm{N}}$ \\
$(-1 / 2,-5 / 2)$ & $-\frac{1}{2} \beta \mathrm{gH}+\frac{5}{4} \mathrm{~A}+\frac{25}{4} \mathrm{Q}+\frac{5}{2} v_{\mathrm{N}}$ \\
$(-1 / 2,-3 / 2)$ & $-\frac{1}{2} \beta \mathrm{gH}+\frac{3}{4} \mathrm{~A}+\frac{9}{4} \mathrm{Q}+\frac{3}{2} v_{\mathrm{N}}$ \\
$(-1 / 2,-1 / 2)$ & $-\frac{1}{2} \beta \mathrm{gH}+\frac{1}{4} \mathrm{~A}+\frac{1}{4} \mathrm{Q}+\frac{1}{2} v_{\mathrm{N}}$ \\
$(-1 / 2,1 / 2)$ & $-\frac{1}{2} \beta g \mathrm{gH}-\frac{1}{4} \mathrm{~A}+\frac{1}{4} \mathrm{Q}-\frac{1}{2} v_{\mathrm{N}}$ \\
$(-1 / 2,3 / 2)$ & $-\frac{1}{2} \beta \mathrm{gH}-\frac{3}{4} \mathrm{~A}+\frac{9}{4} \mathrm{Q}-\frac{3}{2} v_{\mathrm{N}}$ \\
$(-1 / 2,5 / 2)$ & $-\frac{1}{2} \beta \mathrm{gH}-\frac{5}{4} \mathrm{~A}+\frac{25}{4} \mathrm{Q}-\frac{5}{2} v_{\mathrm{N}}$
\end{tabular}

Table 3.3. Levels and energies for an $\mathrm{S}=1 / 2, \mathrm{I}=5 / 2$ spin system. The results in this table are based on the energy level diagram in Fig. 3.6.

\begin{tabular}{|c|c|}
\hline Transition & ENDOR Frequencies \\
\hline$(1 / 2,5 / 2) \Leftrightarrow(1 / 2,3 / 2)$ & $\frac{1}{2} \mathrm{~A}-v_{\mathrm{N}}+4 \mathrm{Q}$ \\
$(1 / 2,3 / 2) \Leftrightarrow(1 / 2,1 / 2)$ & $\frac{1}{2} \mathrm{~A}-v_{\mathrm{N}}+2 \mathrm{Q}$ \\
$(1 / 2,1 / 2) \Leftrightarrow(1 / 2,-1 / 2)$ & $\frac{1}{2} \mathrm{~A}-v_{\mathrm{N}}$ \\
$(1 / 2,-3 / 2) \Leftrightarrow(1 / 2,-5 / 2)$ & $\frac{1}{2} \mathrm{~A}-v_{\mathrm{N}}-2 \mathrm{Q}$ \\
& $\frac{1}{2} \mathrm{~A}-v_{\mathrm{N}}-4 \mathrm{Q}$ \\
$(-1 / 2,5 / 2) \Leftrightarrow(-1 / 2,3 / 2)$ & \\
$(-1 / 2,3 / 2) \Leftrightarrow(-1 / 2,1 / 2)$ & $\frac{1}{2} \mathrm{~A}+v_{\mathrm{N}}-4 \mathrm{Q}$ \\
$(-1 / 2,1 / 2) \Leftrightarrow(-1 / 2,-1 / 2)$ & $\frac{1}{2} \mathrm{~A}+v_{\mathrm{N}}-2 \mathrm{Q}$ \\
$(-1 / 2,-1 / 2) \Leftrightarrow(-1 / 2,-3 / 2)$ & $\frac{1}{2} \mathrm{~A}+v_{\mathrm{N}}$ \\
$(-1 / 2,-3 / 2) \Leftrightarrow(-1 / 2,-5 / 2)$ & $\frac{1}{2} \mathrm{~A}+v_{\mathrm{N}}+2 \mathrm{Q}$ \\
\end{tabular}

Table 3.4. Frequencies for ENDOR transitions associated with an $\mathrm{S}=1 / 2$, $\mathrm{I}=5 / 2$ spin system. The results in this table are based on the energies in Table 3.3. 
Table 3.4 gives the ENDOR frequencies corresponding to an $\mathrm{S}=1 / 2, \mathrm{I}=5 / 2$ system with nuclear quadrupole included. Two sets of five ENDOR lines are predicted, and this agrees with the experimental data in Fig. 3.5. The separation between the middle line of one set and the middle line of the other set is $2 v_{\mathrm{N}}$ and these two middle lines are centered on $\mathrm{A} / 2$. These results support the assignment of $a{ }^{27} \mathrm{Al}$ nucleus as the responsible nucleus in the ENDOR spectrum shown in Fig. 3.5. Since no other spin manifolds (i.e., $\mathrm{m}_{\mathrm{S}}$ values) were observed in the ENDOR spectra, the defect in Fig. 3.3 has $\mathrm{S}=1 / 2$.

The ENDOR spectrum in Fig. 3.5 has an angular dependence. The positions of the ENDOR lines change as the field is rotated from the $c$ axis to the basal plane, but the positions do not change when the magnetic field remains within the basal plane during a rotation. This demonstrates that the hyperfine and nuclear electric quadrupole matrices are axial, with the unique axis oriented parallel to the $c$ axis. The following spin Hamiltonian (with $\mathrm{S}=1 / 2, \mathrm{I}=5 / 2$ ) describes the EPR and ENDOR spectra in Fig. 3.3 and Fig. 3.5 and the angular dependence in Fig. 3.7.

$$
\hat{H}=\beta \mathbf{B} \cdot \overrightarrow{\mathbf{g}} \cdot \hat{\mathbf{S}}+\hat{\mathbf{I}} \cdot \overrightarrow{\mathbf{A}} \cdot \hat{\mathbf{S}}+\hat{\mathbf{I}} \cdot \overrightarrow{\mathbf{Q}} \cdot \hat{\mathbf{I}}-\mathrm{g}_{\mathrm{N}} \beta_{\mathrm{N}} \mathbf{B} \cdot \hat{\mathbf{I}}
$$

Only three parameters $\left(\mathrm{A}_{\|}, \mathrm{A}_{\perp}\right.$, and $\left.\mathrm{P}\right)$ are needed for this axial system. The $\mathrm{Q}$ matrix is traceless with $\mathrm{Q}_{\mathrm{xx}}=-\mathrm{P}, \mathrm{Q}_{\mathrm{yy}}=-\mathrm{P}$, and $\mathrm{Q}_{\mathrm{zz}}=2 \mathrm{P}$. The parameter $\mathrm{P}$ is defined as $\mathrm{e}^{2} \mathrm{qQ} /[4 \mathrm{I}(2 \mathrm{I}-1)]$, where eq is the electric field gradient and $\mathrm{Q}$ is the nuclear quadrupole moment. A least squares fitting program (written in MATLAB), with the data points in Fig. 3.7 as input, gave $\mathrm{A}_{\|}=111.30 \mathrm{MHz}, \mathrm{A}_{\perp}=54.19 \mathrm{MHz}$, and $\mathrm{P}=0.289 \mathrm{MHz}$. The solid curves in Fig. 3.7 were then generated using these "best fit" values of the parameters. Only the relative signs of the hyperfine and nuclear quadrupole parameters could be determined, and a positive sign has been assigned to $\mathrm{A}_{\|}$because the nuclear $\mathrm{g}$ factor for ${ }^{27} \mathrm{Al}$ is positive. The most striking feature of this large aluminum hyperfine interaction is its significant anisotropy. 


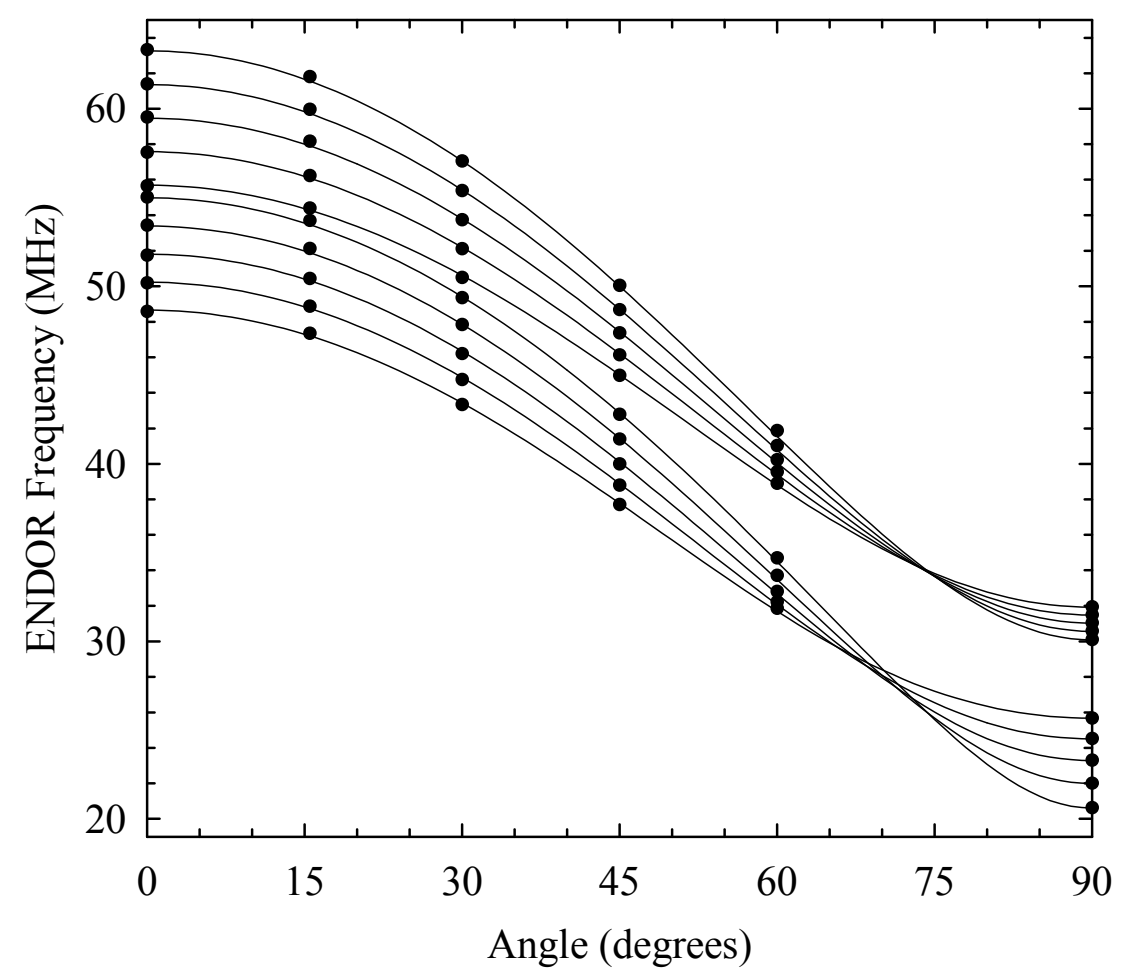

Figure 3.7. Angular dependence of the primary aluminum ENDOR spectrum. The magnetic field was rotated from the $c$ axis $\left(0^{\circ}\right)$ to the basal plane $\left(90^{\circ}\right)$. The solid lines are calculated using the best fit parameters and the discrete points represent experimental data.

\subsubsection{EI-EPR of the Axial ${ }^{27} \mathrm{Al}$ Neighbor}

ENDOR-induced EPR (EI-EPR) is an experimental technique that can be used to extract one EPR spectrum from a set of overlapping EPR spectra. In the present study, EI-EPR is used to verify that the ENDOR spectrum in Fig. 3.5 is associated with the EPR spectrum in Fig. 3.3 and that it arises from the $\mathrm{I}=5 / 2{ }^{27} \mathrm{Al}$ nucleus. When performing an EI-EPR experiment, the frequency of the rf source is set at the peak of an ENDOR signal and is then kept constant as the magnetic field is slowly varied. Thus, EI-EPR is opposite to ENDOR, where the magnetic field is kept constant and the rf frequency is slowly varied. EI-EPR is analogous to taking ENDOR data at a series of discrete values of magnetic field, and then plotting the intensity of a specific ENDOR line as a function of 
magnetic field. EI-EPR spectra are presented as absorption curves, rather than first derivatives as is the case with EPR and ENDOR data.

A complication arises when doing EI-EPR because the position of an ENDOR line depends on the magnetic field intensity. More specifically, the value of the nuclear resonance frequency $\left(v_{N}\right)$ depends on the magnitude of the magnetic field. If $A / 2>v_{N}$, then sets of ENDOR lines will be centered on $A / 2$, and the separation of $2 v_{N}$ between the sets will increase with increasing magnetic field. If $A / 2<v_{N}$, then sets of ENDOR lines will be separated by A and centered on $v_{\mathrm{N}}$. The ENDOR lines move with field in both cases. Thus, it is necessary to know the identity of the responsible nucleus of the ENDOR signal in order to optimize EI-EPR signals. In practice, the operator provides the spectrometer control software with the identity of the nucleus and the direction in which the rf frequency should change with increasing magnetic field. The control software uses this data to shift (to first order) the monitored ENDOR frequency as the magnetic field sweeps. If these parameters are not correct the resulting EI-EPR spectrum will appear distorted. There are, however, other sources of distortion in EI-EPR. For example, if the ENDOR spectrum is complex and contains multiple resonances from different nuclei, these signals may cross each other as the field is swept, thus distorting the ENDOR spectrum at certain fields and distorting the resulting EI-EPR spectrum.

Using the assignment of ${ }^{27} \mathrm{Al}$ as the responsible nucleus for the ENDOR spectrum in Fig. 3.5, EI-EPR data were taken to obtain more information about the underlying structure of the EPR signal. The resulting EI-EPR spectra, obtained by using the ENDOR signals associated with the axial ${ }^{27} \mathrm{Al}$, confirms the source of the unusual shape of the EPR signal. Figure 3.8 shows EI-EPR spectra (solid lines) obtained while setting on three of the ENDOR lines in the lower frequency set in Fig. 3.5. The frequencies corresponding to these three ENDOR lines are included in Fig. 3.8. Only EI-EPR from three lines is necessary to reconstruct the original EPR spectrum because the data from the other two ENDOR lines would be redundant. Each EI-EPR curve represents two 


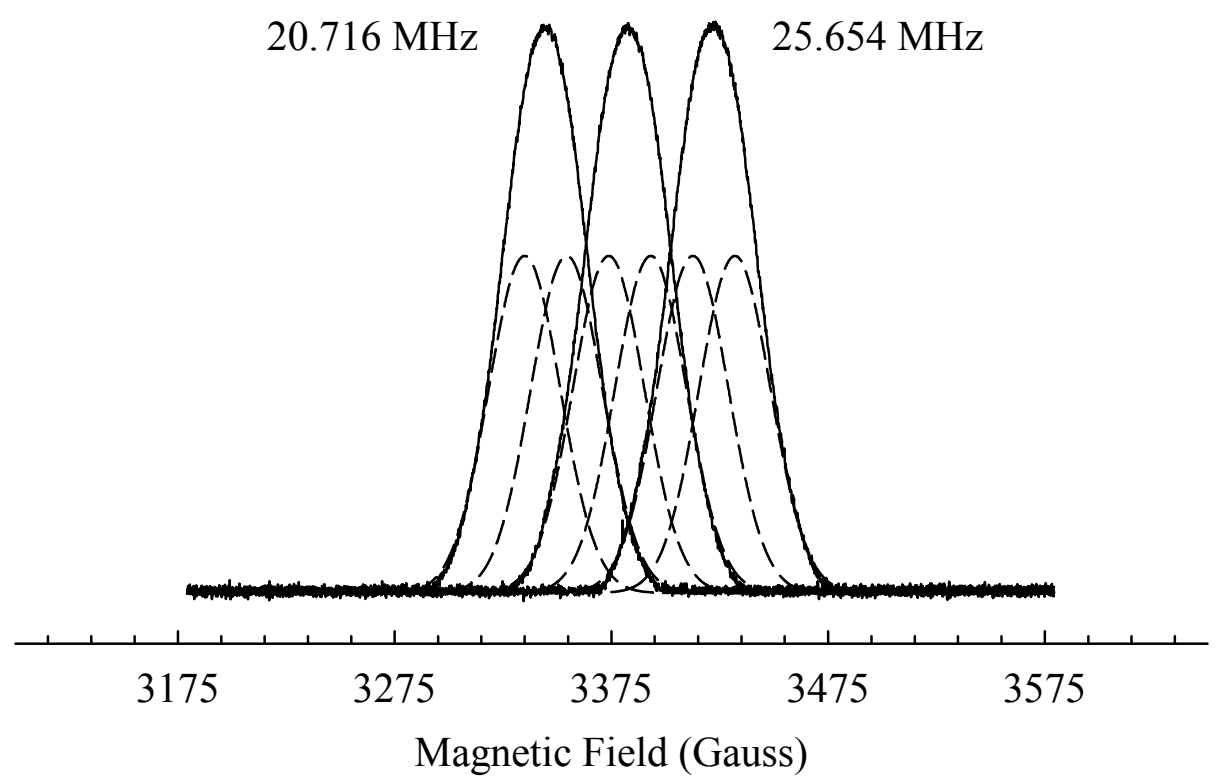

Figure 3.8. EI-EPR data (solid curves) taken using three ENDOR signals of the axial ${ }^{27} \mathrm{Al}$ neighbor. Spectra were taken at $20 \mathrm{~K}$ with the magnetic field aligned perpendicular to the $c$ axis. Each solid curve represents two unresolved EPR transitions (dashed curves).

unresolved EPR signals in Fig. 3.8. The dashed lines are computer-generated Gaussian curves that represent the resolved EPR signals. Six total lines can be counted across the spectrum, thus confirming the $\mathrm{I}=5 / 2$ assignment.

\subsubsection{Additional ENDOR at Low Frequency}

In addition to the large hyperfine interaction with the one aluminum neighbor along the $c$ axis, the EPR spectrum in Fig. 3.3 has weaker equivalent hyperfine interactions with the three aluminum neighbors in the basal plane. Figure 3.9 shows the ENDOR spectrum for the basal-plane neighbors. With the magnetic field parallel to the $c$ axis, the three nearest-neighbor aluminum nuclei in the basal plane have equivalent hyperfine interactions, as verified by the observation of two sets of ENDOR lines located 
at $\mathrm{A} / 2 \pm v_{\mathrm{N}}$ (each set contains five nuclear-quadrupole-split lines). The centers of the two sets are at 5.34 and $12.85 \mathrm{MHz}$ for a magnetic field of $3381 \mathrm{G}$. The separation of 7.51 $\mathrm{MHz}$ is clearly in agreement with the expected ${ }^{27} \mathrm{Al}$ value of $7.51 \mathrm{MHz}$ for $2 v_{\mathrm{N}}$ at this field. EI-EPR was used to verify that all ten components within these two sets of lines were associated with the EPR spectrum shown in Fig. 3.3(a). The EI-EPR experiments also showed that other weak $c$ axis ENDOR lines appearing in the 7.4 to $15 \mathrm{MHz}$ region were due to one or more separate broad EPR signals underlying the primary EPR signal.

For an arbitrary direction of magnetic field in the basal plane, the three weaker aluminum interactions are inequivalent, giving two sets of five ENDOR lines for each nucleus. When the magnetic field was rotated in the basal plane, ENDOR lines from all three basal-plane aluminums were observed and the centers of the high-frequency group (i.e., the five-line sets that correspond to $\mathrm{A} / 2+v_{\mathrm{N}}$ ) stayed between 8 and $13 \mathrm{MHz}$. The
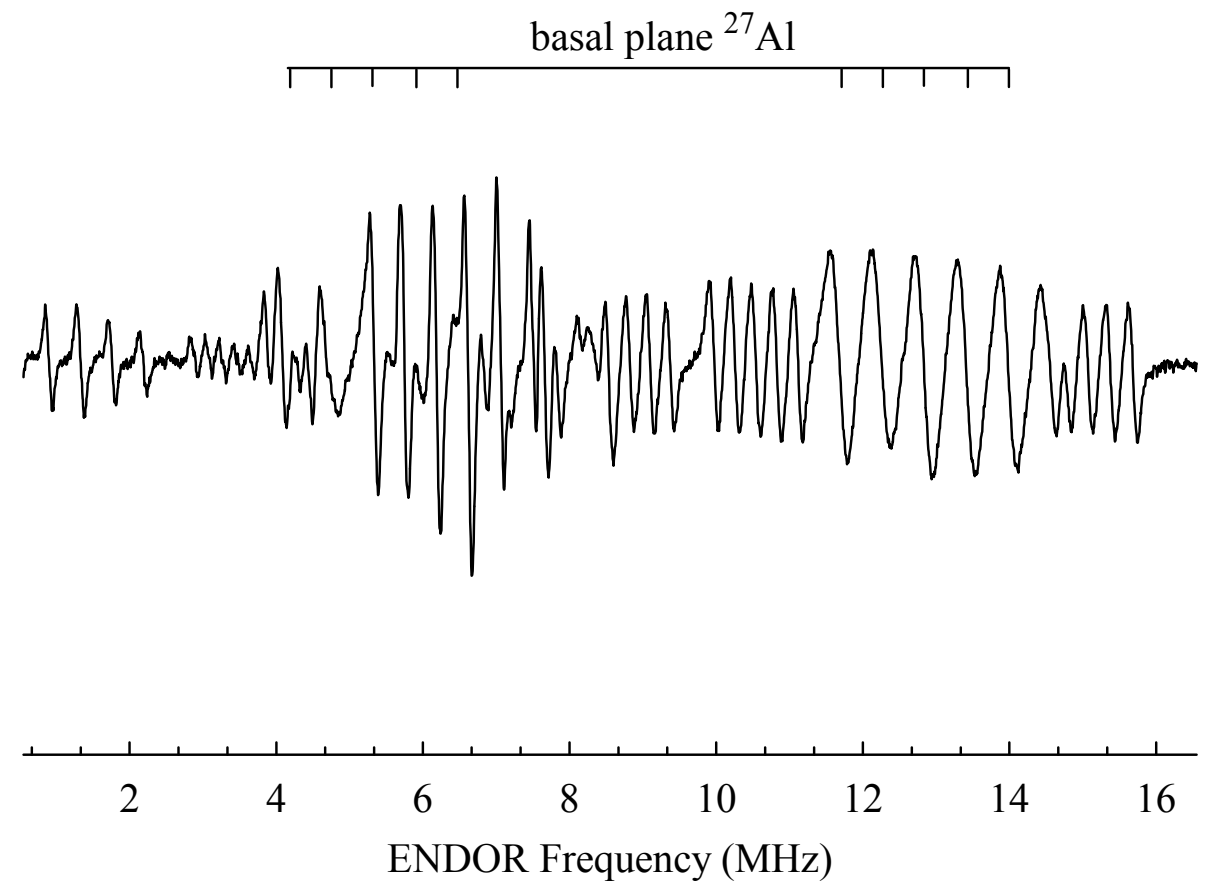

Figure 3.9. ENDOR spectrum of the basal plane ${ }^{27} \mathrm{Al}$ neighbors associated with the donor defect EPR signal. These data were taken at $38 \mathrm{~K}$ with the magnetic field $(3381 \mathrm{G})$ parallel to the $c$ axis. 
ENDOR spectrum repeated itself every $60^{\circ}$. There are two orientations of magnetic field where two of the three five-line ENDOR sets become degenerate, along a crystal axis (the $\mathrm{X}$ direction in Fig. 3.2) and halfway between two crystal axes (the Y direction in Fig. 3.2). The basal plane angular dependence of the three high-frequency sets of quadrupolesplit ENDOR lines reflects the equivalency of the three basal plane aluminum nuclei. The high-frequency groups stayed below $13 \mathrm{MHz}$ when the magnetic field was rotated from the $c$ axis to the basal plane. These results from the two rotation planes suggest that the principal values of the hyperfine matrices describing the interactions with the aluminum neighbors in the basal plane are in the 8 to $20 \mathrm{MHz}$ range.

\subsection{Conclusions}

The ENDOR results presented in this chapter have demonstrated that the defect associated with the EPR signal in Fig. 3.3 has a strong interaction with an axial aluminum nucleus and weaker interactions with three basal plane aluminum nuclei. This suggests that the responsible defect is located at a nitrogen site within the lattice, as shown in Fig. 3.10 , and thus is a donor. Substitutional oxygen impurities or nitrogen vacancies are the most likely candidates. The neutral charge state of the substitutional oxygen donor $\left(\mathrm{O}_{\mathrm{N}}^{0}\right)$ and the neutral charge state of the nitrogen vacancy donor $\left(\mathrm{V}_{\mathrm{N}}^{0}\right)$ are both expected to be paramagnetic with $\mathrm{S}=1 / 2$.

Before irradiation with $\mathrm{x}$ rays, the majority of these donors in the crystal were in the nonparamagnetic singly ionized charge state (either $\mathrm{V}_{\mathrm{O}}^{+}$or $\mathrm{V}_{\mathrm{N}}^{+}$). Only a relatively small portion of the donors were in the neutral charge state, thus accounting for the weak EPR signal observed in the as-grown crystals. Since a crystal must always be electrically neutral, these singly ionized donors in the as-grown crystals are most likely compensated by singly ionized or doubly ionized aluminum vacancy acceptors $\left(\mathrm{V}_{\mathrm{Al}}^{-}\right.$and/or $\left.\mathrm{V}_{\mathrm{Al}}^{2-}\right)$. Exposure to $\mathrm{x}$ rays (ionizing radiation) at room temperature then converts the singly ionized donors to the paramagnetic neutral state by trapping a radiation-induced electron 


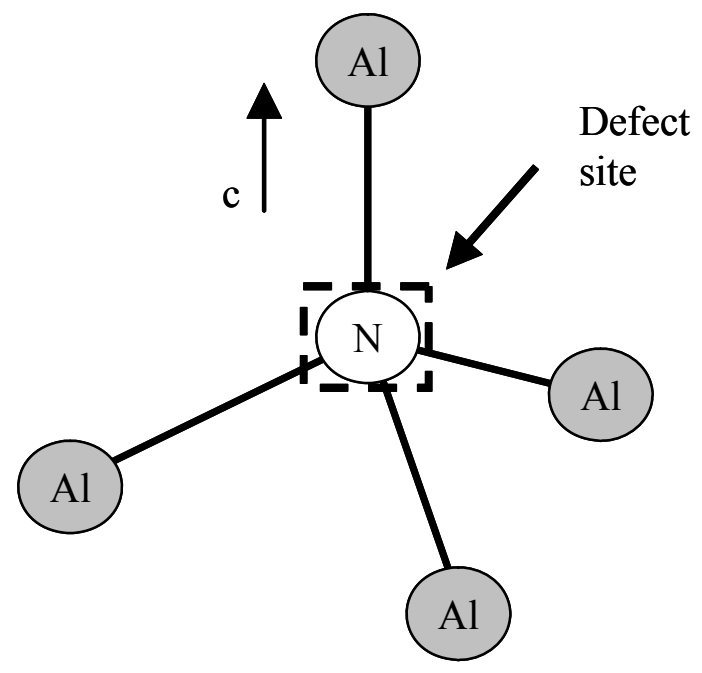

Figure 3.10. Diagram of the location of the defect. There is a primary hyperfine interaction with the axial aluminum atom, and three equivalent interactions with the basal plane aluminum atoms.

at the donor, while the corresponding radiation-induced hole is trapped at an acceptor elsewhere in the crystal. The identity of the trapping site for the holes is not known, although the most likely possibilities are the singly and doubly ionized aluminum vacancies, which would convert to the $\mathrm{V}_{\mathrm{Al}}^{0}$ and $\mathrm{V}_{\mathrm{Al}}^{-}$charge states upon capturing a hole.

From the EPR and ENDOR data, it is difficult to prove whether the defect responsible for the spectrum in Fig. 3.3 is a substitutional oxygen or a nitrogen vacancy. The ${ }^{16} \mathrm{O}$ and ${ }^{18} \mathrm{O}$ isotopes of oxygen together are $99.962 \%$ abundant with $\mathrm{I}=0$, thus ENDOR would not be expected to be observed unless the crystals were isotopically enriched during growth with the ${ }^{17} \mathrm{O}$ isotope. Determination of a responsible nucleus from EPR or ENDOR requires that the nucleus have a nuclear spin to produce a hyperfine interaction and have significant abundance. This means that the only ENDOR expected for both defect models would be from the neighboring aluminum and nitrogen nuclei.

The distribution of the unpaired spin within the defect provides information about 
the possible model. In the case of the nitrogen vacancy, the unpaired spin would be shared by the four neighboring aluminum ions, with a possibility that the axial aluminum ion might have a slightly greater portion of the spin density than the three basal plane ions. This spreading of the unpaired spin over the nearest-neighbor cations is a typical behavior for electrons trapped at anion vacancies, such as the oxygen vacancy in zinc oxide. ${ }^{25}$ For the oxygen vacancy in $\mathrm{ZnO}$, the hyperfine splittings from the axial ${ }^{67} \mathrm{Zn}$ nucleus and nonaxial ${ }^{67} \mathrm{Zn}$ nuclei are $20 \mathrm{G}$ ( $56 \mathrm{MHz}$ ) and $28 \mathrm{G}(78.4 \mathrm{MHz})$, respectively, when the magnetic field is parallel to the $c$ axis. ${ }^{25}$ For $\mathrm{ZnO}$, the difference of $8 \mathrm{G}(22.4$ $\mathrm{MHz}$ ) between the hyperfine values for the axial and nonaxial nuclei is a relatively small percentage (40\%) of the axial value when the field is parallel to the $c$ axis. For AlN, the difference between the hyperfine values for the axial and nonaxial ${ }^{27} \mathrm{Al}$ nuclei is about 33 $\mathrm{G}(92 \mathrm{MHz})$ when the field is along the $c$ axis, which is a much larger percentage (83\%) of the axial value of $39.8 \mathrm{G}(111.3 \mathrm{MHz})$. This comparison with $\mathrm{ZnO}$ suggests that the nitrogen-vacancy model in AIN is not consistent with the observed hyperfine results.

The substitutional oxygen model is consistent with the observed hyperfine results. The electronic configuration of oxygen is $2 s^{2} 2 p^{4}$. An oxygen atom substituting for a nitrogen atom $\left(2 s^{2} 2 p^{3}\right)$ brings with it one extra electron. With this unpaired electron in a p orbital along the $c$ axis of the crystal, the interaction with the axial aluminum nucleus will be considerably larger than the interaction with the three basal plane aluminum nuclei. The isotropic and anisotropic portions of this interaction are both expected to be larger for the axial neighboring nucleus because the lobe of the $\mathrm{p}$ orbital will lie in close proximity to this nucleus. Although absolute evidence does not exist, the observed hyperfine interactions clearly favor the model of an unpaired electron in a p orbital on an oxygen ion. Computational modeling of the hyperfine interactions with neighboring aluminum nuclei for the two separate defects (i.e., the nitrogen vacancy and the substitutional oxygen) is expected to help distinguish between the two models.

In summary, a broad EPR signal has been observed in as-grown single crystals of 


\begin{abstract}
AlN. Exposure to ionizing radiation at room temperature significantly increases the concentration of the defect and causes the crystal to have a deeper reddish color. The paramagnetic center exhibits the axial symmetry of the lattice, and ENDOR reveals a large hyperfine interaction with an axial aluminum neighbor and weaker equivalent hyperfine interactions with three basal plane aluminum neighbors. The present study suggests that the defect responsible for the observed EPR and ENDOR spectra is a neutral donor. The isolated substitutional oxygen $\mathrm{O}_{\mathrm{N}}^{0}$ or the isolated nitrogen vacancy $\mathrm{V}_{\mathrm{N}}^{0}$ are possible models, with the isolated substitutional oxygen being the most likely candidate.
\end{abstract}




\section{CHAPTER 4 \\ Silicon Donors in Aluminum Nitride}

\subsection{Introduction}

Aluminum nitride and its alloys $\left(\mathrm{Al}_{\mathrm{x}} \mathrm{Ga}_{1-\mathrm{x}} \mathrm{N}\right)$ are of considerable interest to the compound semiconductor industry for use in electronic and optoelectronic devices. When applications require n-type group-III nitride material, silicon is often the donor dopant of choice. ${ }^{62}$ Many aspects of this donor impurity, however, are not well understood. In particular, there is a debate about whether silicon in aluminum nitride acts as a shallow effective mass donor, or does it lower its energy by formation of a DX center and become a deep donor. A brief introduction to DX centers is provided in the next section. For more details, the book Deep Centers in Semiconductors, edited by S. T. Pantelides $^{63}$ is a good source of information on DX centers and related subjects.

\subsubsection{Background of DX Centers}

The label of "DX center" was first used by Lang et al. ${ }^{63,64}$ in a study of Te-doped $\mathrm{Al}_{\mathrm{x}} \mathrm{Ga}_{1-\mathrm{x}} \mathrm{As}$. This name was proposed as a short-hand description of their proposed explanation of the experimental results. The relation of the persistent photoconductivity in the sample and the donor concentration indicated the presence of a donor, which is the " $D$ " in the name. However, because the center's concentration was not consistently proportional to the donor concentration, these investigators concluded that the center must be a complex of a donor and some other unidentified defect " $\mathrm{X}$ ". Thus, the center was named a "DX center." In this model, a configuration coordinate diagram and Stoke's shifts are used to explain the difference between the energy necessary to thermally excite an electron to the conduction band and the energy to photoexcite an electron to the conduction band. Although subsequent hydrostatic pressure experiments showed that these DX centers are isolated donors ${ }^{63}$ and not a complex of a donor and some other unknown defect, "DX center" persists. The DX center is now explained as an isolated 
donor that lowers its energy by lattice relaxation. For a DX center this lattice relaxation is usually quite large, and a configuration coordinate diagram is still used to describe transitions.

A DX center is typically associated with a persistent photoconductivity, a Stoke's shifting of the energy required to thermally excite and optically excite electrons to the conduction band, and a negative $U$ behavior. ${ }^{63}$ Negative $U$ behavior is associated with large lattice relaxation. When a defect center traps an electron, there is a change in energy U. Under normal circumstances, meaning no large lattice relaxation, this energy change, $\mathrm{U}$, is positive. The positive change in energy is a result of repulsion arising from the electron-electron interaction. If the lattice undergoes a distortion after trapping an electron, the energy will undergo an additional change. The total energy change of the defect state is then the increase due to the trapping of the electron minus the decrease in energy due to the lattice distortion. If the energy change, due to the lattice distortion, is larger than the energy change from the capture of an electron, then the overall change in energy is negative and thus the name "negative U center."

A negative U behavior allows for "self compensation" of a defect. For example, consider a donor in an otherwise perfect lattice that has three charge states corresponding to two captured electrons, one captured electron, and no captured electrons. Without the lattice relaxation, the charge state with no trapped electrons would be the lowest energy state and the state with two trapped electrons would be the highest energy state. If the large lattice relaxation lowers the energy enough, the charge state with two trapped electrons may have its energy below that of the state with only one trapped electron. If this happens, the one-electron charge state can lower its energy by either losing an electron or trapping an electron. This results in the no-electron and two-electron states being populated at the same time while the one-electron state is not populated. This self compensation arising from negative $U$ behavior is the reason why the negative- $U$ concept was introduced in the first place. Anderson initially proposed the negative- $U$ concept to 
explain the lack of paramagnetism in chalcogenide glasses. For EPR studies, when a defect exhibiting negative-U behavior is in its lowest energy configuration, there are no states with an unpaired spin, and thus there is no EPR signal present.

\subsubsection{Silicon in III-Nitrides}

DX centers have been studied for several decades now, starting initially with Se, Te, $\mathrm{Si}$, and $\mathrm{Sn}$ donors in $\mathrm{AlGaAs}{ }^{63-68}$ and $\mathrm{S}$ and Te donors in GaAsP. ${ }^{69,70}$ In recent years, theoretical and experimental studies have been performed on $\mathrm{GaN}, \mathrm{AlN}$, and $\mathrm{Al}_{\mathrm{x}} \mathrm{Ga}_{1-\mathrm{x}} \mathrm{N}$ alloys. $^{3-5,71,72,73-76}$ The studies relevant to the work presented in this chapter are those pertaining to silicon. Silicon has gained interest as a dopant that produces $n$ type material, but the microscopic structure of the defect is not completely understood. Some investigators have suggested that silicon forms a DX center in AlN and in AlGaN alloys above certain Al concentrations, ${ }^{3,71-75}$ while other investigators have suggested that silicon is a shallow effective mass donor in AlN. ${ }^{5}$

In AlN, silicon substitutes for aluminum in the lattice. A silicon atom has one more electron than an aluminum atom and is thus a donor in AlN. Silicon donors have three possible charge states. A silicon atom has four electrons available for bonding, but only three are needed when silicon replaces aluminum in this III-V lattice. This leaves one unpaired spin for the neutral charge state, $\mathrm{Si}_{\mathrm{Al}}{ }^{2}$. The neutral state is paramagnetic and an EPR signal is observed. For the singly ionized charge state, $\mathrm{Si}_{\mathrm{Al}}^{+}$, one electron has been removed and three electrons remain for bonding. The $\mathrm{Si}_{\mathrm{Al}}^{+}$charge state is not paramagnetic, and an EPR signal is not expected. The third charge state, $\mathrm{Si}_{\mathrm{Al}}^{-}$, is less likely than the other two, but should also be considered. This negative charge state occurs if the neutral donor traps an additional electron, thus resulting in two extra electrons beyond the three needed for bonding. These two extra electrons are expected to pair off and leave a nonparamagnetic center. To compare with the example mentioned in the previous section, the singly ionized positively charge state would be the state with no 
trapped electrons, the neutral charge state would be the state with one trapped electron, and the negatively charged state would be the state with two trapped, but paired, electrons.

In 1998, Van de Walle ${ }^{5}$ conducted a theoretical study of the formation of DX centers in $\mathrm{Al}_{\mathrm{X}} \mathrm{Ga}_{1-\mathrm{x}} \mathrm{N}$ for $\mathrm{x}=0$ and $\mathrm{x}=1$. His calculations suggested that the $\mathrm{DX}$ center was not a stable configuration for silicon in AIN. In AlGaAs alloys, silicon is known to form a DX center. ${ }^{63}$ Van de Walle suggests that the lack of $\mathrm{DX}$ formation in $\mathrm{Al}_{\mathrm{X}} \mathrm{Ga}_{1-\mathrm{x}} \mathrm{N}$ is due to the fact that $\mathrm{AlGaN}$ alloys have smaller lattice constants and larger elastic constants than AlGaAs alloys. In 2000, Zeisel et $a l^{71}$ performed an experimental study of silicon in AlN thin films. This investigation explored the conductivity of the sample and characterized an EPR signal from the silicon. The results of their study demonstrated that samples of AlN doped with silicon show persistent photoconductivity, exhibit a difference between the thermal activation and photoexcitation of the conductivity, and have EPR behavior that could be explained if silicon is a DX center.

If silicon is a shallow donor in a material with no other defects, or if the Fermi level is high enough, then silicon would remain in the neutral charge state and an EPR signal would is expected to be present without the need for photoexcitation. If the material is compensated (i.e., a significant concentration of acceptors is present), then the neutral silicon would lose an electron to an acceptor and be in the nonparamagnetic singly ionized positive charge state.

\subsection{Experimental Results}

The results presented in this chapter consist of optical absorption and EPR data from two samples of bulk, single crystal aluminum nitride. One sample was intentionally doped with silicon during growth and had a concentration of silicon exceeding approximately $10^{18} \mathrm{~cm}^{-3}$. The other sample was not deliberately doped, but the presence of silicon was verified by the observation of an EPR signal associated with silicon ${ }^{71}$ as 
well as by subsequent elemental analysis. The two samples were cut from larger boules that were grown at Crystal IS (Green Island, NY) and then sent to West Virginia University for characterization. The dimensions of the intentionally doped sample were approximately $3 \times 6.5 \times 0.62 \mathrm{~mm}^{3}$, and the dimensions of the undoped sample were approximately $3 \times 5 \times 0.26 \mathrm{~mm}^{3}$. All the EPR data were taken at $9.48 \mathrm{GHz}$ on a Bruker EMX EPR spectrometer. An Oxford Instruments ESR-900 helium-gas-flow system was used to control the sample temperature during the EPR measurements. Values of the static magnetic field were obtained with a proton gaussmeter. Optical absorption data were taken at room temperature using a Cary-14 uv-vis-nir spectrophotometer (modernized by Olis in Bogart, GA).

\subsubsection{Optical Absorption and EPR Signal}

Although both contain silicon, the behaviors of the undoped and doped samples were measurably different. The EPR signal in the doped sample closely resembles the EPR signal previously observed in highly doped thin films of AlN. ${ }^{3,71,72}$ In contrast, the EPR signal in the undoped sample has a significantly different behavior. The work described in this Chapter is still in progress, and the continuation of these studies in the future will hopefully resolve questions presently being raised.

Figure 4.1 shows the optical absorption of each sample taken at room temperature. The undoped sample has a significant peak at $440 \mathrm{~nm}$ with a small peak at $250 \mathrm{~nm}$. The doped sample is dominated by a large absorption below $300 \mathrm{~nm}$ with a small peak at $440 \mathrm{~nm}$ on the tail of the dominating band.

The two samples also have differing EPR behavior. At room temperature, the undoped sample shows a sharp EPR signal with a width of about 1.5 Gauss occurring near $\mathrm{g}=1.9905$. This spectrum in shown in Fig. 4.2(a). At room temperature, light has

no observable effect on the intensity or shape of this EPR signal. As the sample is cooled in the dark, the signal broadens and is not observable for temperatures below $100 \mathrm{~K}$ while 

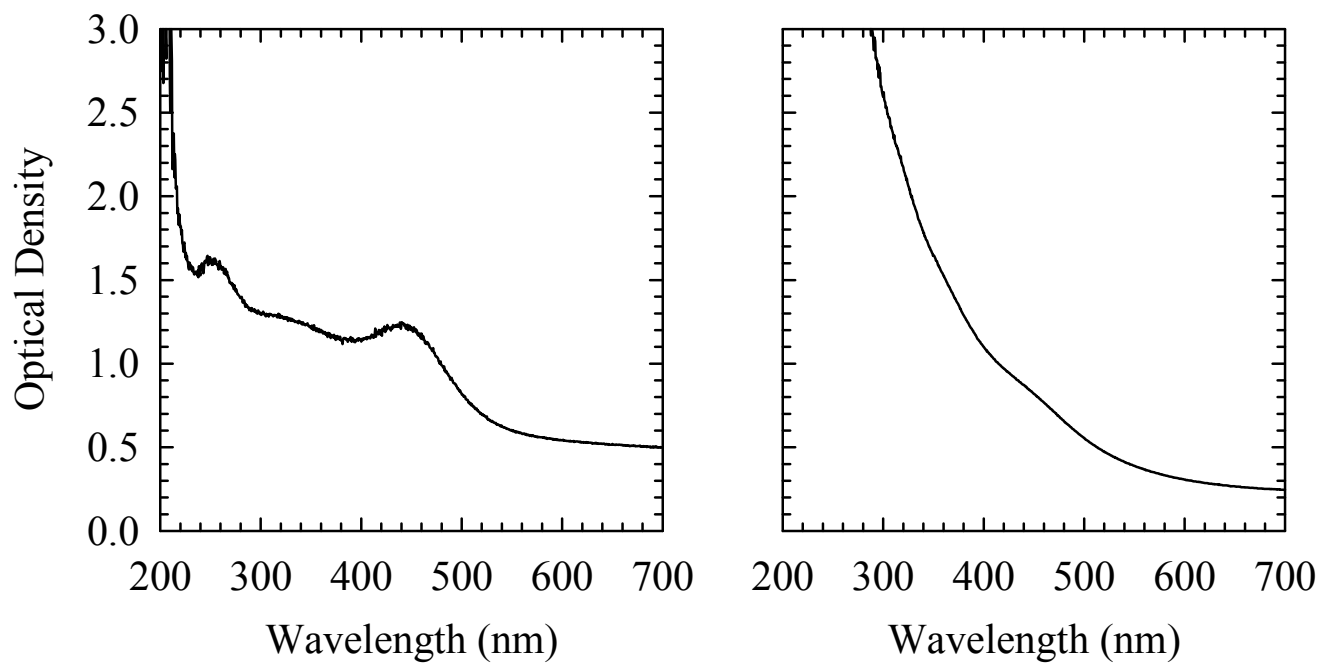

Figure 4.1. Comparison of the optical absorption of undoped (left) and doped (right) samples.

still in the dark. At low temperatures, photoexcitation causes this EPR signal to become much larger, as shown in Fig. 4.2(b) for illumination at $20 \mathrm{~K}$. The g values at room temperature and low temperature are the same within experimental error and agree with the previously reported $g$ value of $g=1.9885$ for the shallow donor silicon EPR signal. ${ }^{3}$ At $20 \mathrm{~K}$, the photoinduced signal has a line width of approximately 12 Gauss. Once the signal is photoinduced at low temperatures, the sample can be restored to its pre-illumination state by warming to room temperature. This recovery process is fast and only requires a few minutes or less at room temperature.

The temperature dependence of the photoinduced effect was explored in the undoped sample for temperatures ranging from 5 to $200 \mathrm{~K}$. For temperatures below 150 $\mathrm{K}$, the silicon EPR signal is not observable without illumination. This is explained by the signal broadening sufficiently that the intensity of the signal is below the noise level. Above $150 \mathrm{~K}$, the EPR is observable without illumination, but still has a photoinduced growth. As the temperature is raised, above $150 \mathrm{~K}$ the pre-illumination intensity of the signal increases while the photoinduced effect when light is turned on decreases. At 

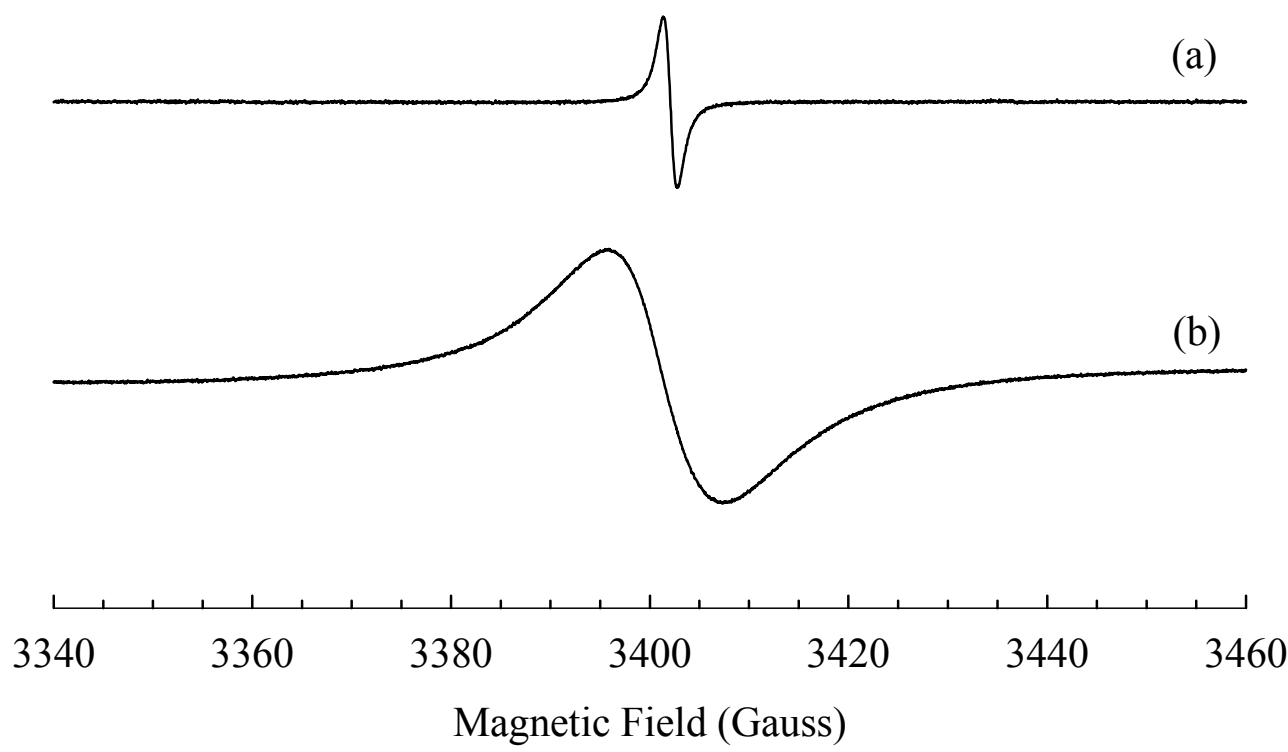

Figure 4.2. EPR spectra taken from the undoped sample (a) at room temperature with no light, and (b) at $20 \mathrm{~K}$ during illumination with $442 \mathrm{~nm}$ light.

room temperature, there is little or no difference in intensity of the EPR signal with and without illumination.

The silicon-doped sample displays a slightly different behavior. At room temperature there is no observable EPR signal with or without light. As the sample is cooled in the dark, the EPR signal is still not observable. When the sample is illuminated with light at $20 \mathrm{~K}$, an EPR signal appears at the same $\mathrm{g}$ value as the signal in the undoped sample. The photoinduced signal in the silicon-doped sample, however, has a much larger intensity than the photoinduced signal in the undoped sample, when compared under similar illumination conditions. At $20 \mathrm{~K}$, the linewidth of this signal is approximately 15 gauss. Figure 4.3 shows the EPR signals for the doped and undoped samples on the same vertical scale for comparison. As with the undoped sample, the photoinduced effect in the doped sample can be annealed out by warming to room temperature. 


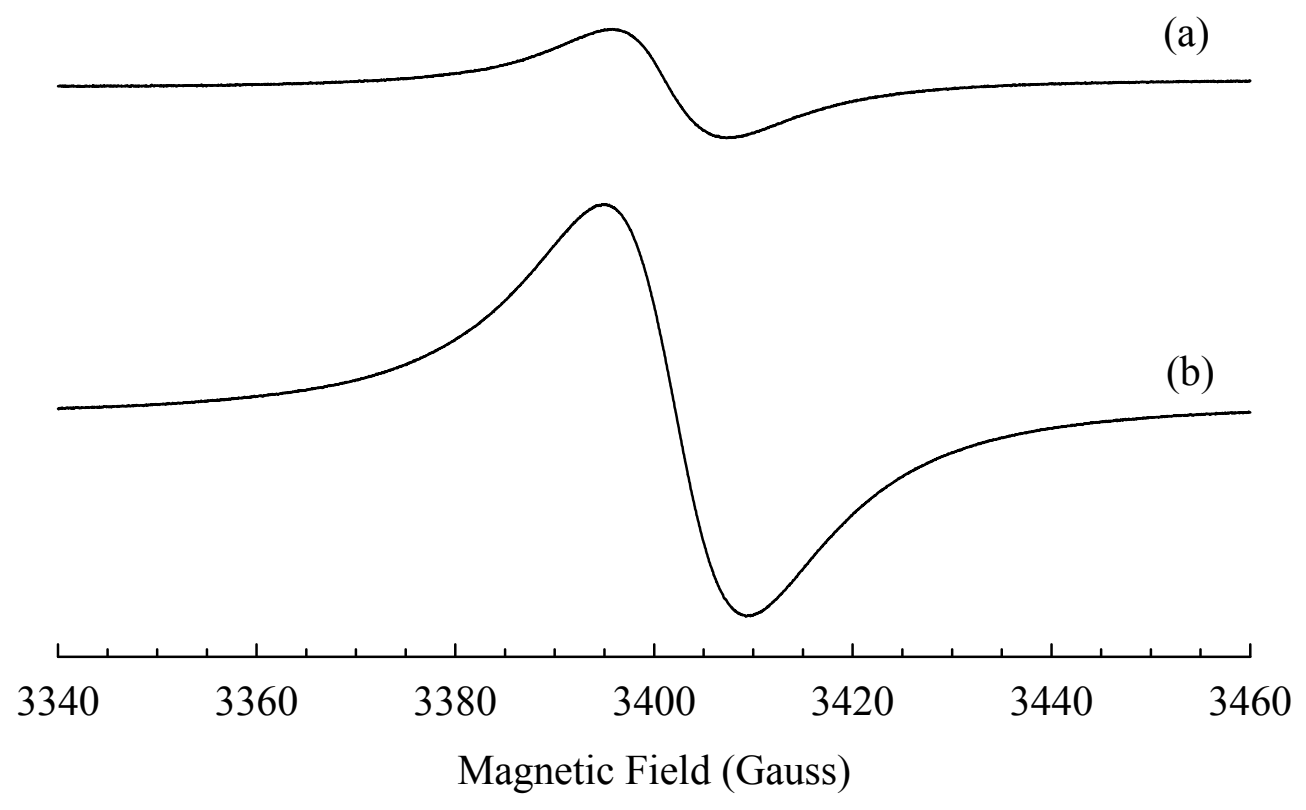

Figure 4.3. EPR of (a) undoped and (b) doped samples under illumination at $20 \mathrm{~K}$ with $442 \mathrm{~nm}$ light.

\subsubsection{Photoexcitation Wavelength Dependence}

The wavelength dependence of the production of the silicon EPR signal was studied in each sample at $35 \mathrm{~K}$. The light source was a xenon arc lamp coupled to a monochromator. The intensity of the light incident on the microwave cavity was adjusted to maintain constant photon flux by using a neutral density filter. The power incident on the sample was $0.74 \mathrm{~mW}$ at $400 \mathrm{~nm}$.

Before taking each data point, the sample was warmed to room temperature to anneal out any photoinduced changes from the previous data point. The sample was then cooled back down to $35 \mathrm{~K}$ in the dark. The EPR spectrometer was set to an operating mode that recorded the signal intensity versus time at a fixed magnetic field. After ten seconds of collecting data with the sample in the dark, the sample was illuminated and the growth of the intensity of the signal was recorded as a function of time. This process was repeated for each subsequent data point. Figure 4.4 is a plot of the intensity of the 

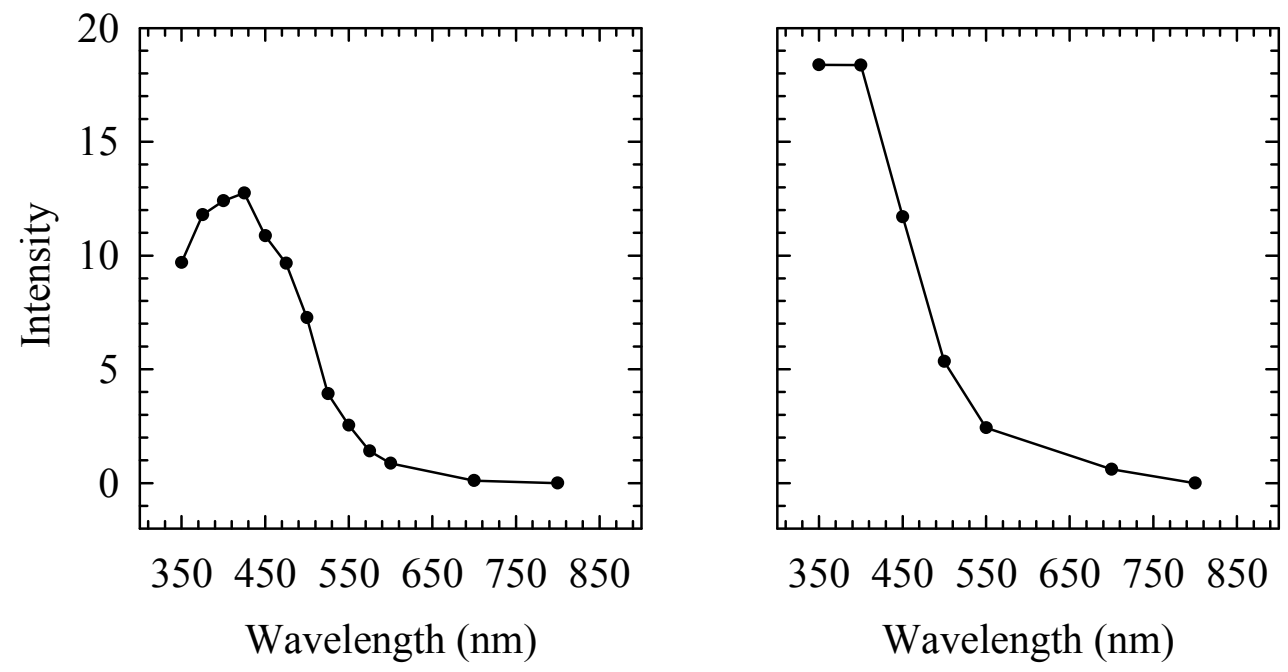

Figure 4.4. Wavelength dependence of the production of the photoinduced silicon EPR signal from the undoped (left) and doped (right) samples.

EPR signal 140 seconds after the light was turned on for each wavelength. The wavelength dependences of the two samples are shown side by side for comparison. For both samples, the signal reaches a maximum around 400 to $425 \mathrm{~nm}$.

\subsubsection{Photoinduced Growth}

The photoinduced growth of the EPR signal in each sample was monitored using the same spectrometer operating mode to record the intensity as a function of time. Figure 4.5 shows the growth of the signal from the two samples taken at $35 \mathrm{~K}$ with 400 $\mathrm{nm}$ light using the xenon lamp and monochromator. The first ten seconds of each plot is without light to establish a baseline. After ten seconds of taking data, the shutter was opened and the sample was illuminated. The undoped sample has a rapid growth. As soon as the light is turned on, the growth in the undoped sample has a large slope that decreases with time. After ten minutes this growth has significantly slowed. The doped sample shows a slightly different behavior. The intensity of the EPR signal in the doped sample doesn't saturate until much longer times, compared to the undoped sample. After ten minutes of illumination, the signal is still growing in the silicon-doped sample. 

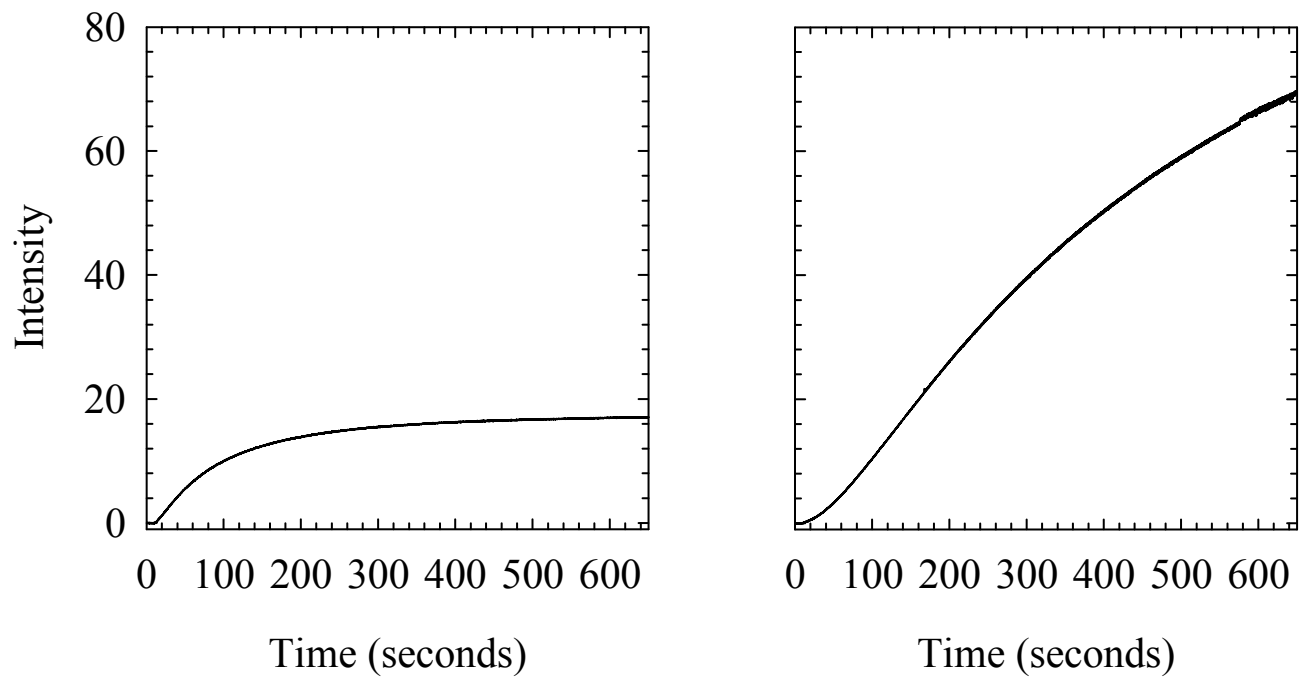

Figure 4.5. Growth curves for the silicon EPR signal in the undoped (left) and doped (right) samples. Data were taken at $35 \mathrm{~K}$ with $400 \mathrm{~nm}$ excitation light at $0.74 \mathrm{~mW}$ power.

\subsubsection{Production and Decay}

During the course of the present study, it was noticed that the intensity of the EPR signal from the undoped sample decreased after the excitation light was removed. To explore this behavior, $442 \mathrm{~nm}$ light from a He-Cd laser was used to quickly produce the EPR signal. Figure 4.6 shows the growth and decay of the EPR signal in both samples at $35 \mathrm{~K}$ when the laser is turned on and then shuttered. The first ten seconds of data were taken while the sample was in the dark to establish a baseline. At the ten-second point, the sample was illuminated. After the intensity of the EPR signal saturated, the light was shuttered. Here, the behavior described earlier is clearly shown. The signal from the undoped sample shows a sharp growth that quickly saturates. Upon shuttering the light, there is a significant and rapid drop in signal intensity. The signal from the doped sample shows a distinctly different behavior. When the light is shuttered, there is some decay but it is small and slow in comparison to the rapid decay of the signal in the undoped 

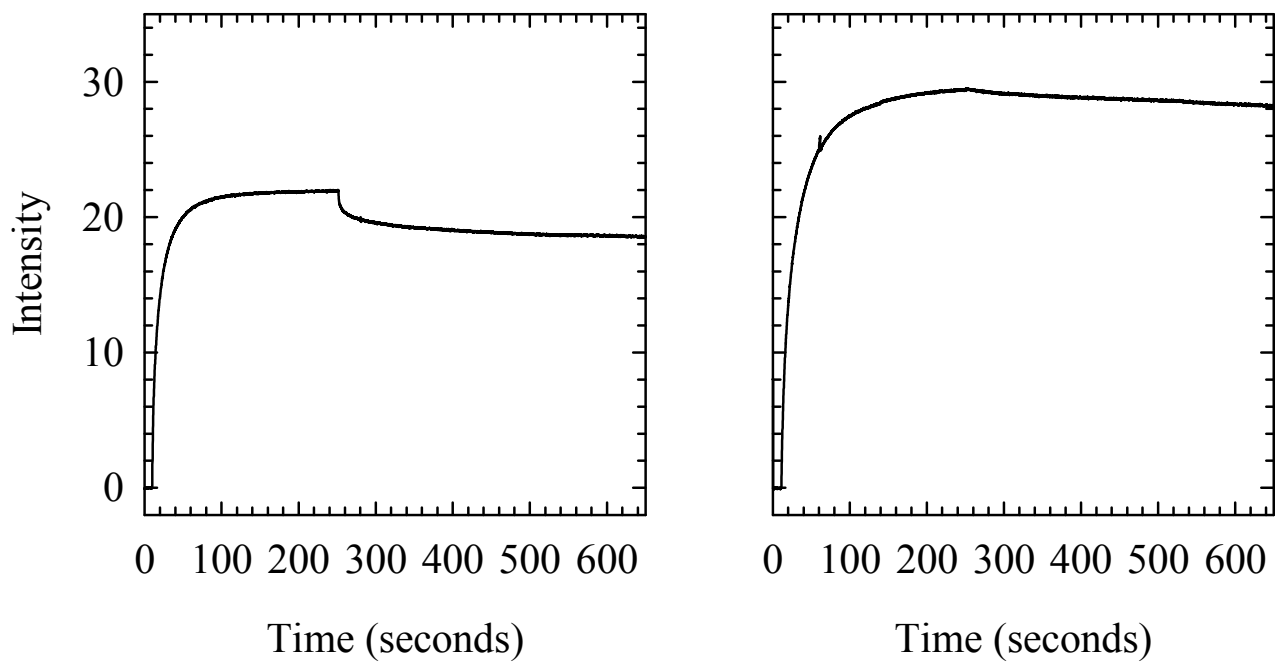

Figure 4.6. Growth and decay curves for the undoped (left) and doped (right) samples. Samples were illuminated at $35 \mathrm{~K}$ with $442 \mathrm{~nm}$ laser light and the light was shuttered once the signal reached saturation.

sample. This behavior of growth and decay was observed for the entire range of temperatures from 10 to $160 \mathrm{~K}$.

\subsubsection{Pulsed Thermal Anneal}

A pulsed thermal anneal study was performed on both of the samples. In this study, the sample is illuminated at low temperature to produce changes in the charge states of the defects. For example, a donor may trap an electron and become paramagnetic. As the sample temperature is raised, the defect gains enough thermal energy for the defect to release the trapped electron. In this study, the silicon EPR signal was photoinduced at $20 \mathrm{~K}$ using $442 \mathrm{~nm}$ light from a $\mathrm{HeCd}$ laser. The light was then shuttered and the intensity was measured after the initial rapid decay was complete. Since there is an initial drop in intensity in the signal from the undoped sample once the light is turned off, data were not taken until some time after the light was removed to ensure that the signal had reached a steady state. The sample was quickly warmed to a set temperature and held at that temperature for two minutes. The sample was then 
quickly cooled back to $20 \mathrm{~K}$ and the signal intensity was recorded. This procedure was repeated for sequentially higher temperatures.

Figure 4.7 shows the data from the pulsed thermal anneal study for both samples. The EPR signal from the doped sample (on the right) decays quickly between 40 and 60 $\mathrm{K}$. This result agrees with the observations of Zeisel et al. ${ }^{71}$ that the signal disappears around $60 \mathrm{~K}$. The undoped sample is a more complex case. The signal appears to be relatively stable below $40 \mathrm{~K}$, however, between 40 and $80 \mathrm{~K}$ the EPR signal decays to about half the initial intensity. When the sample is heated to temperatures between 80 and $120 \mathrm{~K}$, the decay is slower. At temperatures above $120 \mathrm{~K}$ the signal once again quickly decays, completely removing the photoinduced effect between 120 and $160 \mathrm{~K}$.

\subsection{Discussion}

The EPR signal from each of the AIN samples has been identified as a shallow silicon donor in a neutral charge state by the g value near 1.9909 . This value is in agreement with the value obtained by Zeisel et al. ${ }^{71}$ The behavior of the EPR signal from each of the two present samples is different. The reasons for this difference in behavior

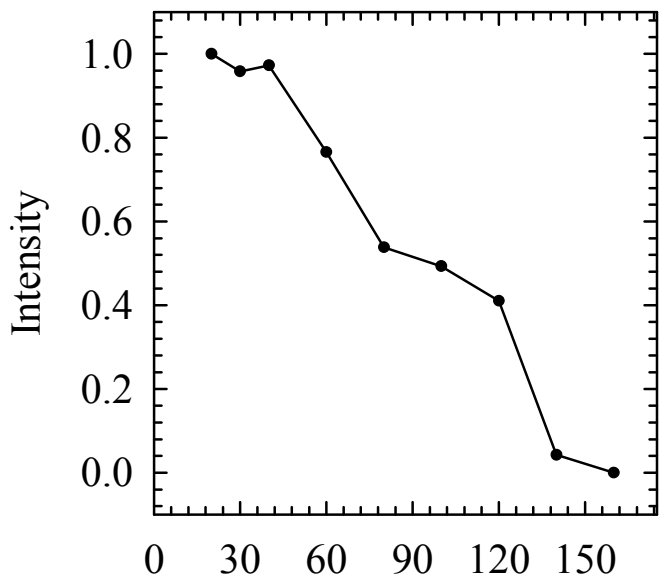

Temperature (K)

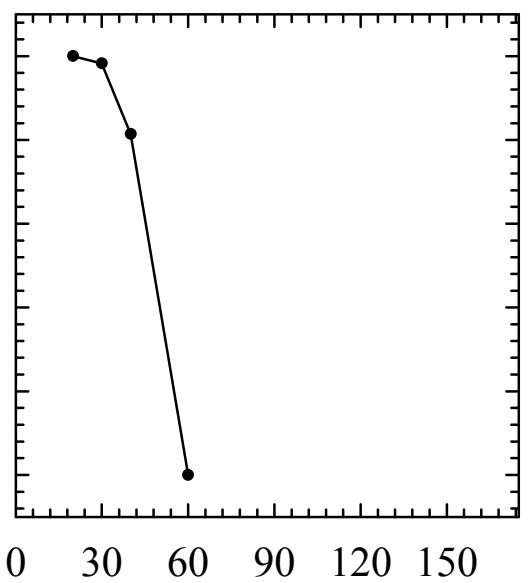

Temperature (K)

Figure 4.7. Pulsed thermal anneal results for the silicon EPR signal in the undoped (left) and doped (right) AlN samples. 
are unclear and are still being investigated. The purpose of this chapter has been to present the data acquired to date and to discuss possible explanations. The behavior of the doped sample is simpler. There is no EPR signal observed at room temperature with or without light, or at low temperature when cooled in the dark. When illuminated at low temperature the silicon EPR signal appears. In the undoped sample, however, an EPR signal is observed at room temperature without the need for any light. As the temperature is lowered with the sample in the dark, the signal broadens until it is no longer observable. At the low temperatures, illumination with light with wavelengths shorter than 500 $\mathrm{nm}$ increases the intensity of the signal and makes it observable once again.

Aluminum nitride is expected to contain significant amounts of other point defects, besides silicon, that could play a significant role in the present observations. It is reasonable to assume the presence of one or more acceptor levels in the band gap. This could explain the behavior of the silicon EPR signal from both samples. Assuming silicon is a shallow donor and acceptors are present in the sample, the acceptor levels would lower the Fermi level and allow electrons from the neutral silicon states to drop to the acceptor states and convert a portion of the acceptors to singly ionized negative charge states. If the concentration of acceptors is greater than that of the concentration of donors, then all the donors would be in a nonparamagnetic state and no EPR signal would be present under dark steady-state conditions. If there are more donors than acceptors, then some of the donors would remain in a paramagnetic state and some would lose their electrons and form a nonparamagnetic state. This corresponds to the case when an EPR signal is present without light, but increases in intensity during illumination.

In the silicon-doped AIN sample, there is no EPR signal observed at room temperature or at low temperature in the dark. This could mean that there simply are more acceptor states than donor states and all the silicon defects are in the singly ionized negative charge state. This behavior can also be explained using the DX center model. ${ }^{71,72}$ If silicon in AIN is a DX center, no EPR signal would be expected when the 
sample is cooled in the dark because the silicon donors would be present in both the singly ionized positive and singly ionized negative charge states simultaneously (both nonparamagnetic). Illumination then converts both types of singly ionized charge states to the shallow neutral charge states and produces an EPR signal. This scenario, however, assumes that there are no other defects in the crystal, or at least that there is no exchange of charge with other defects.

There is also the matter of the room temperature behavior of the undoped AlN sample. In this sample, there is a silicon EPR signal present at room temperature without illumination, but no observable EPR signal at low temperature. As stated before, the silicon EPR signal broadens as the temperature is lowered. The lack of an observable signal at low temperature could simply mean that there are still silicon donors in the neutral charge state, and the resulting EPR signal is too broad to be observed above the noise. The presence of an EPR signal in the absence of illumination is consistent with silicon being a non-DX shallow donor, if the donor concentration is higher than the acceptor concentration. Under this scenario, the lack of a silicon EPR signal in the doped sample would require a much larger concentration of acceptors than the undoped sample since the doped sample has a much larger concentration of silicon. This large variation of acceptor concentration is, of course, possible since the two samples are from different boules. Examination of samples where the acceptor concentrations are better known may prove useful in clarifying this situation. The undoped sample clearly raises the following question about the DX center model for silicon. Why is there a silicon EPR signal present without illumination in the undoped sample if all the silicon defects are expected to be in nonparamagnetic charge states?

Another interesting behavior of these EPR signals is their initial decay after the light is turned off at low temperature. In previous studies, the silicon EPR signal was reported to be stable after the light was removed. ${ }^{3,71,72}$ This is the case for the doped sample; however, this is not the case for the undoped sample, at least for a portion of the 
signal. Once the silicon EPR signal is produced in the undoped sample using light, the EPR signal intensity will quickly decrease by about $15-20 \%$ in a matter of a few minutes when the light is removed. This occurs for any temperature where the EPR signal is affected by light. It is possible, if the concentration of defects is sufficiently large, that some donors and some acceptors are in close physical proximity within the crystal. When the distance between the donor and acceptor is small, the electron may be able to "tunnel" from the donor to the acceptor without the need to go through the conduction band. This would require that the donor concentration, the acceptor concentration, or both concentrations be large. When comparing the EPR signal intensity in the undoped sample to that of the silicon-doped sample (which does not have this quick decay), it seems unlikely that the donor concentration would be large enough to lead to the tunneling mechanism described above. However, if the concentration of acceptors is larger than that of the donors, the silicon donors would be fully compensated and no EPR signal would be expected without illumination.

The observed behavior of the doped sample raises questions about the possibility of donors and acceptors recombining in the undoped sample by tunneling. If silicon is a non-DX shallow donor in AlN, the undoped sample must contain acceptors. Furthermore, the concentration of donors must be greater than the concentration of acceptors, and the combined concentration of silicon donors and acceptors must be large to allow for tunneling. The relative intensities of the silicon EPR signals from the two samples (see Fig. 4.3) suggest that the doped sample has a larger concentration of silicon impurities than the undoped sample. There is no EPR signal in the doped sample in the absence of light, thus the concentration of silicon donors must be less than the concentration of available acceptors in the doped sample. The doped sample would be expected to exhibit more tunneling than the undoped sample because there is a greater concentration of silicon donors and acceptors in the doped sample than the undoped sample. Experimentally, however, there is little, if any, initial decay in the EPR signal of 
the doped sample once the light is removed. This observation is not consistent with the tunneling explanation used above to explain the initial decay in the EPR signal of the undoped sample, once the light is removed.

The last interesting set of data comes from the thermal anneal study. Again, the silicon-doped sample behaves in much the same way as previously reported., ${ }^{3,71,72}$ Once the silicon EPR signal is photoinduced in the doped sample at low temperatures, the signal persists until the sample is warmed to between 40 and $60 \mathrm{~K}$. Above $60 \mathrm{~K}$, the signal decays to its pre-illumination state. In the shallow donor model, the trapped electron on the neutral silicon donor is thermally ionized into the conduction band and then becomes trapped at an acceptor. The DX center model would describe this in terms of the thermal energy required to overcome the barrier in the configuration coordinate diagram associated with the conversion of the $\mathrm{d}^{0}$ (the neutral donor state without the lattice relaxation) to the $\mathrm{DX}^{-}$state. The undoped sample is very different. The EPR signal in the undoped sample also begins to decay in the temperature range between 40 and $80 \mathrm{~K}$, but the signal intensity is only reduced to about half of the intensity existing before beginning the warming process. In the range between 80 and $120 \mathrm{~K}$ the signal intensity has very little decay. When the sample is warmed above $120 \mathrm{~K}$, the intensity drops rapidly again and returns to its pre-illumination value when annealed to $160 \mathrm{~K}$.

The behavior of silicon in the two samples is unexpected and confusing. In the simplest view of silicon in AlN as a shallow donor, the singly ionized positive silicon substitutional traps an electron, converting it to the paramagnetic neutral charge state, when illuminated with wavelengths below $550 \mathrm{~nm}$. At low temperatures, there is not enough thermal energy to excite the trapped electron back to the conduction band, thus the EPR signal is stable. When the temperature is sufficiently raised, it is expected that the electron is thermally excited to the conduction band and retrapped by some acceptor. It would also be expected that this would happen for all the isolated silicon substitutional defects in the crystal, converting all the defects to their pre-illumination state, thus 
leaving no observable EPR once again. The data from the doped sample supports this model; however, this model does not explain the behavior of the undoped sample.

Further work is needed on silicon in aluminum nitride to answer these and other questions about the nature of this donor and the behavior it exhibits. 


\section{CHAPTER 5}

\section{Oxygen Vacancies and Zinc Vacancies in Zinc Oxide}

\subsection{Introduction}

\subsubsection{Crystal Structure and Symmetry of ZnO}

Zinc oxide $(\mathrm{ZnO})$ is a II-VI compound semiconductor with the same hexagonal crystal lattice and wurtzite crystal structure as aluminum nitride. $\mathrm{ZnO}$, having the wurtzite structure, belongs to the $\mathrm{P}_{3} \mathrm{mc}$ space group. ${ }^{52}$ For the unit cell of $\mathrm{ZnO}$, refer to the unit cell of AlN shown in Fig 3.1. In Fig 3.1, the positions of the Al and $\mathrm{N}$ sites are analogous to positions of the $\mathrm{Zn}$ and $\mathrm{O}$ sites, respectively. The structural differences between $\mathrm{AlN}$ and $\mathrm{ZnO}$ lie in the dimensions of the unit cell and the lengths of bonds.

The lattice parameters for the unit cell of $\mathrm{ZnO}$ are $a=3.2496 \AA$ and $c=5.2042$ $\AA .^{77}$ The crystal structure is defined by the same four atom basis as AlN. Starting with zinc at the origin, the positions of the remaining atoms in the unit cell are given in Table 5.1 as fractions of the lattice constant in the corresponding direction.

\begin{tabular}{|c|c|c|}
\hline Atom & Position (a,a,c) & Position (a,a,c) \\
\hline $\mathrm{Zn}$ & $(0,0,0)$ & $(0.5,0.5,0.5)$ \\
\hline $\mathrm{O}$ & $(0,0,0.3819)$ & $(0.5,0.5,0.8819)$ \\
\hline
\end{tabular}

Table 5.1. Positions of basis atoms in a unit cell of $\mathrm{ZnO}$.

$\mathrm{ZnO}$ deviates slightly from the ideal wurtzite structure and has a value of 0.3819 for the parameter $u .^{77}$ Note that the $\mathrm{c} / \mathrm{a}$ ratio of 1.6018 for $\mathrm{ZnO}$ differs by a small amount from the 1.600 value of AlN and from the perfect wurtzite value of 1.633. These crystallographic results for $\mathrm{ZnO}$ translate into bond lengths of $1.9875 \AA$ for the axial neighbor and $1.9743 \AA$ for the three basal plane neighbors. The band gap of $\mathrm{ZnO}$ is approximately $3.372 \mathrm{eV}$ at room temperature. It is a direct-band-gap material. 


\subsubsection{Growth of Bulk ZnO Crystals}

Currently, the three main methods used to grow bulk single crystals of zinc oxide are hydrothermal, high pressure melt, and chemical vapor transport. ${ }^{52}$ Each of these methods have advantages and limitations. In general, it is important to know how a crystal was grown, since this information allows the researcher to better appreciate the relative types of point defects that may be present. A comprehensive review paper on past studies of $\mathrm{ZnO}$, including growth techniques of bulk single-crystal $\mathrm{ZnO}$, has been published by Ozgur et $a .^{52}$

The hydrothermal technique is used commercially by companies such as Tokyo Denpa in Japan. ${ }^{78}$ In this method, a small single crystal of $\mathrm{ZnO}$ is used as a seed, and the growth vessel is a steel autoclave with an inert metal liner (such as gold or platinum). The seed is supported in the upper half of the autoclave by a platinum wire. Nutrient, consisting of small polycrystalline pieces of $\mathrm{ZnO}$, is placed in the lower half of the autoclave, and the autoclave is then partially filled with water containing a small amount of mineralizer, $\mathrm{KOH}(3 \mathrm{~mol} / \mathrm{l})$ and $\mathrm{LiOH}(1 \mathrm{~mol} / \mathrm{l})$. The autoclave is then sealed. The temperature in the lower part of the autoclave, containing the nutrient, is held at a value slightly higher than in the upper part of the autoclave, containing the seed crystals. Programmable temperature controllers precisely determine these temperatures as a function of time. Nutrient is dissolved by the mineralizer, material is then transported to the upper portion of the autoclave, and deposits onto the cooler seed. The temperature gradient between the upper and lower portions of the autoclave determines the growth rate. This hydrothermal growth process takes anywhere from weeks to months to grow large boules. During growth, temperatures typically range from 300 to $400{ }^{\circ} \mathrm{C}$ with pressures of 70 to $100 \mathrm{MPa}$.

Advantages of the hydrothermal method include high crystal quality and large boules. Limitations of the hydrothermal method include nonuniform distribution of impurities and large concentrations of lithium and hydrogen. The lithium compensates 
group-III donors and results in crystals with significantly reduced carrier concentrations (i.e., that are nearly semi-insulating). ${ }^{79}$ Lithium in $\mathrm{ZnO}$ is an acceptor, and thus has been proposed in recent years as a possible dopant to produce p-type material. ${ }^{52}$

High-pressure melt growth is another method used to produce commercial $\mathrm{ZnO}$ bulk crystals. Cermet, a company in Atlanta, Georgia, employs this technique. ${ }^{80}$ Cermet's high-pressure melt growth method uses an RF induction heater and a pressurized growth vessel. $\mathrm{ZnO}$ powder is loaded into a crucible, and the RF generator produces large currents and associated joule heating. At high enough temperature and pressure, the $\mathrm{ZnO}$ powder melts (around $1900{ }^{\circ} \mathrm{C}$ ). Once a melt is formed, the crucible is either slowly lowered out of the heated region to allow crystallization or a seed crystal is dipped into the melted and slowly pulled up while rotating.

Chemical vapor transport produces high quality $\mathrm{ZnO}$ crystals. $\mathrm{ZnO}$ powder is used as the source and is placed at one end of a horizontal tube with a seed crystal placed at the other end. The end containing the powder is heated to about $1150{ }^{\circ} \mathrm{C}$. The end containing the seed is kept at a lower temperature of about $1100{ }^{\circ} \mathrm{C}$. The low vapor pressure of $\mathrm{Zn}$ and $\mathrm{O}$ at these temperatures makes a carrier gas necessary to transport the material to the cooler end. Hydrogen gas is normally used for this task. In the hotter end, the $\mathrm{ZnO}$ reacts with the hydrogen gas to form $\mathrm{Zn}$ vapor and water vapor. When the vapor reaches the cooler end, the reverse reaction occurs. Newly formed $\mathrm{ZnO}$ crystallizes on the seed to make a larger single crystal. Growth runs lasting from 150 to 175 hours will produce two-inch-diameter wafers approximately $1 \mathrm{~cm}$ thick. Samples grown by this technique may contain hydrogen, iron, nickel, cobalt, and manganese impurities.

\subsubsection{Previous Studies of Vacancies in $\mathrm{ZnO}$}

The role of oxygen vacancies and zinc vacancies in $\mathrm{ZnO}$ continues to be a topic of considerable interest. ${ }^{81-89}$ These native defects can exist in more than one charge state, and they are often invoked to explain the observed optical and electrical properties of 
$\mathrm{ZnO}^{52}$ As bulk crystals, thin films, and nanoparticles of $\mathrm{ZnO}$ are produced and studied today, it is important to develop a more detailed understanding of these basic native defects. Electron paramagnetic resonance (EPR) is well suited for this task since it provides a direct method to monitor the paramagnetic charge states of vacancies in $\mathrm{ZnO}$, and thus complements other experimental techniques such as optical absorption, photoluminescence, and positron annihilation. EPR signals from vacancies in $\mathrm{ZnO}$ are sharp and easily distinguished because these centers are deep within the band gap (i.e., localized) and have minimal hyperfine interactions that cause broadening $\left({ }^{67} \mathrm{Zn}\right.$ is $4.1 \%$ abundant with $\mathrm{I}=5 / 2$ and ${ }^{17} \mathrm{O}$ is $0.037 \%$ abundant with $\mathrm{I}=5 / 2$ ).

In 1970, Smith and Vehse ${ }^{25}$ used EPR to observe the singly ionized charge state of oxygen vacancies $\left(\mathrm{V}_{\mathrm{O}}^{+}\right.$) (often referred to as an $\mathrm{F}^{+}$center) in a bulk $\mathrm{ZnO}$ crystal that had been irradiated with high-energy electrons of energy of $0.6,1.8$, and $2.0 \mathrm{MeV}$. EPR was observed only for the samples irradiated with 1.8 and $2.0 \mathrm{MeV}$. In the past, the $\mathrm{g}=$ 1.96 shallow donor EPR signal has been misidentified as the oxygen vacancy. ${ }^{28,29}$ The signal observed by Smith and Vehse occurred at $\mathrm{g}_{\|}=1.9948$ and $\mathrm{g}_{\perp}=1.9963$, and was greatly enhanced by visible light. These investigators detected and measured hyperfine interactions with the four neighboring ${ }^{67} \mathrm{Zn}$ nuclei, and thus conclusively proved that isolated singly ionized oxygen vacancies were being monitored. ${ }^{25}$ Additional EPR studies quickly followed this initial work. The threshold energy for oxygen displacement was investigated by irradiating $\mathrm{ZnO}$ crystals with electron beams having different energies, ${ }^{90,91}$ and a comprehensive angular-dependence study of the resolved hyperfine associated with the singly ionized oxygen vacancy provided the complete ${ }^{67} \mathrm{Zn}$ hyperfine and nuclear electric quadrupole matrices for the nearest neighbors. ${ }^{26}$ Later EPR studies explored photoinduced changes in charge state of oxygen vacancies. ${ }^{27,91,117,118}$

Also in 1970, Taylor et al. ${ }^{34}$ and Galland and Herve ${ }^{22}$ used EPR to observe singly ionized zinc vacancies $\left(\mathrm{V}_{\mathrm{Zn}}^{-}\right.$) in $\mathrm{ZnO}$ crystals after irradiations with high-energy neutrons and electrons, respectively. These spectra had principal $g$ values ranging from 2.0024 to 
2.0193. Singly ionized zinc vacancies are deep acceptors in $\mathrm{ZnO}$, and EPR shows that the hole (the unpaired spin) is localized on a single oxygen neighbor. This gives rise to two distinct versions of the singly ionized zinc vacancy that are easily distinguished by EPR. The vacancy having the hole localized on the oxygen neighbor located along the $c$ axis is referred to as the axial center, while a vacancy having the hole localized on one of the three oxygen neighbors in the basal plane is referred to as a nonaxial center. Models are shown in Fig. 5.1 for these zinc vacancies. In Fig. 5.1, a hole is schematically represented by the symbol $\oplus$.

In the initial studies of zinc vacancies in $\mathrm{ZnO}$, both groups ${ }^{22,34}$ observed the EPR signal from the nonaxial form of the isolated singly ionized zinc vacancy. Galland and Herve $^{22}$ also detected signals from the isolated axial singly ionized zinc vacancy and the neutral zinc vacancy $\left(\mathrm{V}_{\mathrm{Zn}}^{0}\right)$. The ground state of the neutral isolated zinc vacancy is an

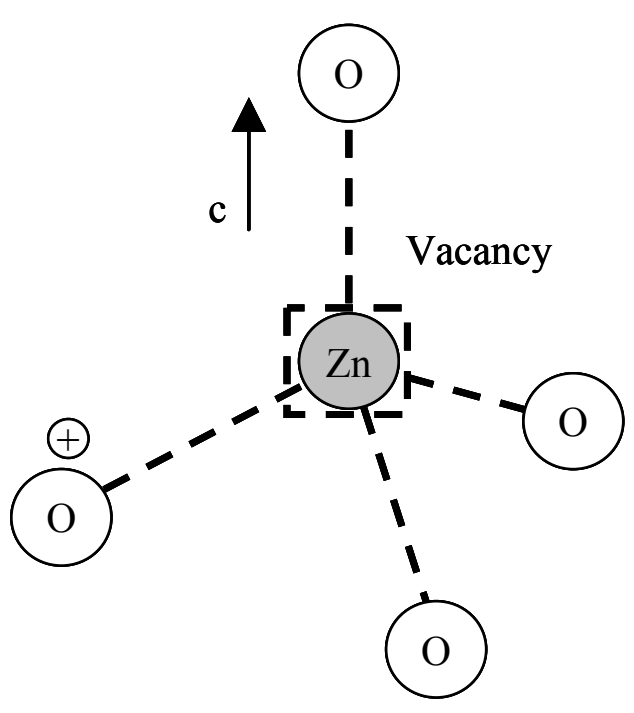

(a)

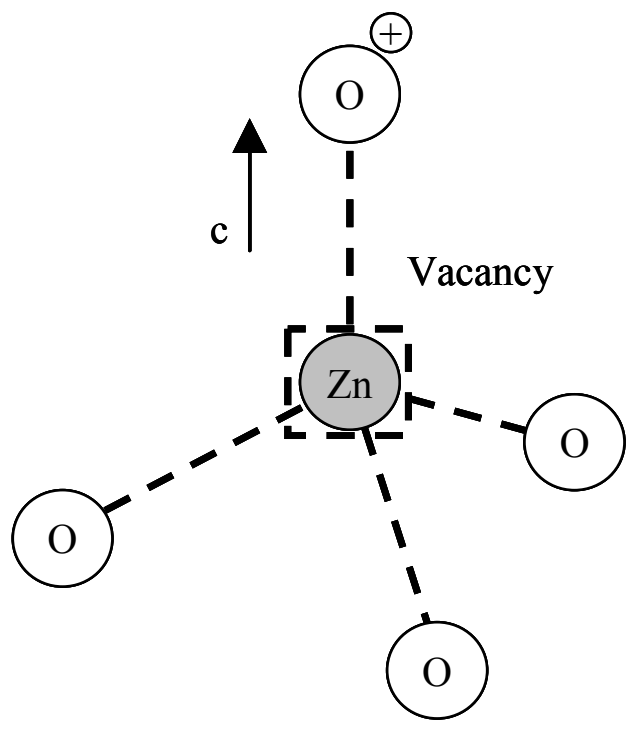

(b)

Figure 5.1. (a) Model of the non-axial zinc vacancy showing the trapped hole on a neighboring basal plane oxygen atom. Note the three possible sites for the trapped hole. (b) Model of the axial zinc vacancy with the hole trapped on the axial oxygen atom. 
$\mathrm{S}=1$ spin system where the two holes are localized separately on two of the nonaxial oxygen neighbors. ${ }^{22}$ Follow-up EPR studies verified the isolated nonaxial singly ionized zinc vacancy and the isolated neutral zinc vacancy assignments, ${ }^{92,93}$ and further explored the thermal activation of the hole as it hops between axial and nonaxial oxygen positions around the isolated zinc vacancy. ${ }^{94}$ Recently, both zinc and oxygen vacancies have been observed in optically detected magnetic resonance spectra obtained from electronirradiated crystals. $^{95-98}$

In this chapter, a comprehensive EPR and optical excitation study of oxygen and zinc vacancies in $\mathrm{ZnO}$ is described. These defects were produced by irradiating a crystal near room temperature with $1.5 \mathrm{MeV}$ electrons. Prior to the irradiation, only a " $\mathrm{g}=1.96$ " EPR signal from shallow donors was observed at low temperature (i.e., no EPR or photoinduced EPR signals from vacancies could be detected in the as-grown crystal). After the irradiation, an EPR signal from $\mathrm{Fe}^{3+}$ impurities was observed. These iron ions were unintentionally present in the crystal, and were in the $\mathrm{Fe}^{2+}$ charge state prior to the irradiation. The presence of the $\mathrm{Fe}^{3+}$ ions in the irradiated crystal proved to be an important feature of the study, as they provided deep donor traps to hold photoexcited electrons when the irradiated crystal was exposed to light while at low temperature. ${ }^{78}$ This work demonstrates an optimum procedure to monitor the low-temperature EPR signals from the oxygen and zinc vacancies and the $\mathrm{Fe}^{3+}$ ions, identifies new EPR signals from zinc vacancies having an $\mathrm{OH}^{-}$ion on an adjacent oxygen site, and uses different wavelengths of light at low temperature to establish the positions of defect states in the energy gap. The results illustrate the interrelated behavior of zinc vacancies, oxygen vacancies, and iron impurity ions when the irradiated crystal is illuminated at low temperature.

\subsection{Experimental Results}

A thin $c$-plate $\mathrm{ZnO}$ crystal grown by the seeded chemical vapor transport (CVT) 
method $^{99}$ at Eagle-Picher (Miami, OK) was used in the present study. This bulk n-type crystal, with dimensions of $3.0 \times 5.0 \times 0.5 \mathrm{~mm}^{3}$, was irradiated for 2 hours with a beam of $1.5 \mathrm{MeV}$ electrons from a van de Graaff accelerator (approximately $2 \times 10^{17}$ electrons were incident on the crystal). The irradiation was performed by Professor L. A. Kappers at the University of Connecticut. During the irradiation, the crystal was mounted on a water-cooled metal block and stayed near room temperature. Before the irradiation, the primary defects in the crystal were shallow Group III donors and deeper $\mathrm{Fe}^{2+}$ donors. The crystal remained $\mathrm{n}$ type after the irradiation, but the concentration of carriers at room temperature decreased from $7 \times 10^{16}$ to $3 \times 10^{13} \mathrm{~cm}^{-3}$ (according to Hall measurements). The EPR data were taken with a Bruker EMX spectrometer operating at $9.48 \mathrm{GHz}$. An Oxford Instruments helium gas flow system provided temperature control. Slots in the end of the rectangular $\mathrm{TE}_{102}$ microwave cavity allowed optical access to the sample while at low temperature. Illumination sources were a He-Cd laser $(10 \mathrm{~mW}$ at $325 \mathrm{~nm})$ and a $150 \mathrm{~W}$ short-arc xenon lamp coupled to a $0.22 \mathrm{~m}$ monochromator (with a $1200 \mathrm{~g} / \mathrm{mm}$ grating blazed at $500 \mathrm{~nm}$ ).

After the electron irradiation, the $\mathrm{ZnO}$ crystal appeared pale yellow to the eye. At room temperature, the broad optical absorption band responsible for this yellow color was found to have a peak near $409 \mathrm{~nm}(3.03 \mathrm{eV})$ and a full width at half maximum of $0.78 \mathrm{eV} .{ }^{100}$ Recently, a much more intense absorption band, with an identical position and shape, was observed in $\mathrm{ZnO}$ crystals heated to $1100{ }^{\circ} \mathrm{C}$ in $\mathrm{Zn}$ vapor, and assigned to the neutral charge state of the oxygen vacancy. ${ }^{81}$ The work presented in this chapter now provides further support for the assignment ${ }^{81}$ of the $409 \mathrm{~nm}$ band to neutral oxygen vacancies, since the EPR spectra described in the following section clearly show that neutral oxygen vacancies are a primary defect produced by the electron irradiation. This near-band-edge optical absorption feature was observed by earlier investigators, ${ }^{101-103}$ but they did not suggest a specific model for the responsible defect. 


\subsubsection{EPR Spectra}

Before being subjected to an electron irradiation, EPR spectra from the sample looked similar to many other as-grown ZnO crystals. The only observable EPR signal present was the shallow donor signal at $\mathrm{g}=1.96$. Figure 5.2 shows the EPR spectra after the electron irradiation for the magnetic field oriented along the $c$ axis (a) and for the magnetic field oriented perpendicular to the $c$ axis (b). These data were taken at $30 \mathrm{~K}$ after the electron irradiation, but before any illumination (the sample was cooled in the dark). The spin-lattice relaxation times of the paramagnetic defects in these spectra are long at $30 \mathrm{~K}$, and their EPR signals are microwave-power saturated and weak when the spectrometer is operated in the conventional manner. To circumvent this problem and greatly enhance the observed signal intensities at low temperature, out-of-phase detection was used for the $100 \mathrm{kHz}$ magnetic field modulation. ${ }^{78,104}$ The signal from isolated $\mathrm{Fe}^{3+}$ ions, ${ }^{78,105}$ near $3375.6 \mathrm{G}$, is observable because the electron irradiation has significantly

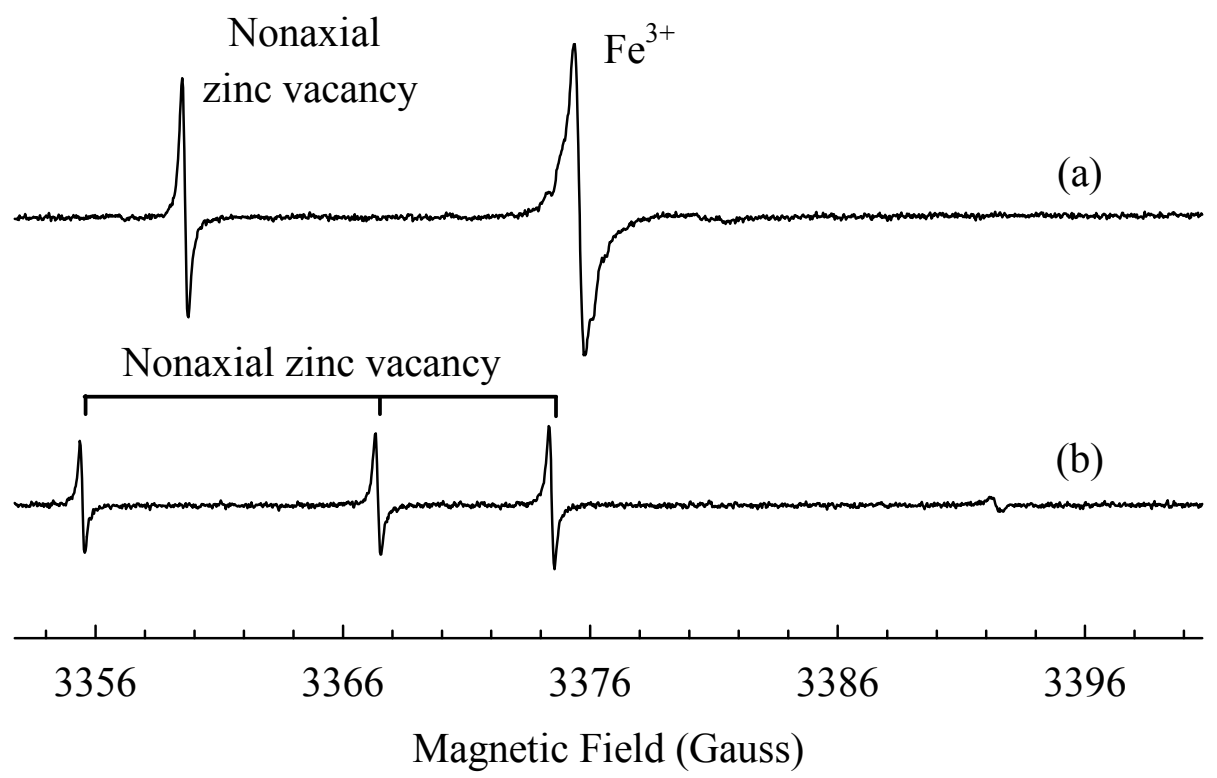

Figure 5.2. EPR spectra taken at $30 \mathrm{~K}$ from a $\mathrm{ZnO}$ crystal irradiated with 1.5 MeV electrons. No illumination was used. The magnetic field was (a) parallel to the $c$ axis and (b) perpendicular to the $c$ axis (at an arbitrary angle in the basal plane). 
lowered the Fermi level. The concentration of $\mathrm{Fe}^{3+}$ ions in the irradiated crystal, determined in separate measurements made in-phase at room temperature, is approximately $2.8 \times 10^{16} \mathrm{~cm}^{-3}$. Also present in Fig. 5.2(a) is the nonaxial singly ionized zinc vacancy $\left(\mathrm{V}_{\mathrm{Zn}}^{-}\right)$at $3359.6 \mathrm{G}$. This latter signal, labeled Center A by Galland and Herve, ${ }^{22}$ represents a zinc vacancy with the hole localized on one of the three nonaxial oxygen neighbors. The observation of a nonaxial singly ionized zinc vacancy signal in Fig. 5.2(a), before illumination, suggests that there were an insufficient number of donors (i.e., iron and shallow Group III ions) available in the crystal to provide the electrons needed to convert all of the isolated zinc vacancies to their doubly ionized form $\left(\mathrm{V}_{\mathrm{Zn}}^{2-}\right)$. The observation of a nonaxial singly ionized zinc vacancy signal before illumination is also consistent with the expectation that the ground state of the isolated doubly ionized isolated zinc vacancy is relatively high in the band gap.

The spectrum in Fig. 5.2(b) was taken with the magnetic field perpendicular to the $c$ axis of the crystal at an arbitrary angle in the basal plane. The three lines at 3351,3363 , and $3370 \mathrm{G}$ are due to nonaxial singly ionized zinc vacancies. When the field is perpendicular to the $c$ axis, the threefold degeneracy of the EPR spectrum shown in Fig. 5.2(a) is lifted and the EPR spectrum now consists of three signals as shown in Fig. 5.2(b). This is expected as there are three possible inequivalent sites for the hole next to the vacancy when the magnetic field is in the basal plane. There are six possible sites total for this defect. When the magnetic field is along the $c$ axis of the crystal, all six sites are equivalent, thus producing a single line as shown in Fig. 5.2(a). When the magnetic field is rotated away from the $c$ axis, all six sites are observable as six distinct EPR lines. When in the basal plane, the sites "pair up," meaning there are three inequivalent pairs of sites. This results in an EPR spectrum consisting of three equally intense lines as shown in Fig. 5.2(b).

Figure 5.3 shows EPR spectra obtained at low temperature after the $\mathrm{ZnO}$ crystal was electron irradiated at room temperature. These data were taken with the magnetic 
field parallel to the $c$ axis. The EPR spectrum in Fig. 5.3(a) was taken after the electron irradiation, but before any illumination (the sample was cooled in the dark). The EPR spectrum in Fig. 5.3(b) was taken after a brief illumination at $30 \mathrm{~K}$ with $325 \mathrm{~nm}$ laser light. For reasons explained later in Section 5.2.2, the sample was illuminated only for a few seconds and the concentrations of the photoinduced defects did not reach equilibrium. The $325 \mathrm{~nm}$ above-band-gap light produces large numbers of electrons and holes, some of which become trapped at existing defects. The $\mathrm{Fe}^{3+}$ ions trap electrons and their EPR signal disappears. [Note: Large zero-field splittings ${ }^{106,107}$ prevent the observation of EPR signals from $\mathrm{Fe}^{2+}$ ions in $\mathrm{ZnO}$ at $9.5 \mathrm{GHz}$.] Some of the holes are trapped at neutral oxygen vacancies, thus causing an EPR signal from singly ionized oxygen vacancies $\left(\mathrm{V}_{\mathrm{O}}^{+}\right)$to appear at $3395.1 \mathrm{G}$. Other holes are trapped at defect complexes consisting of a zinc vacancy and an impurity ion (either hydrogen or a Group III donor), and give rise to two EPR signals at 3357.6 and $3358.7 \mathrm{G}$ and four EPR signals

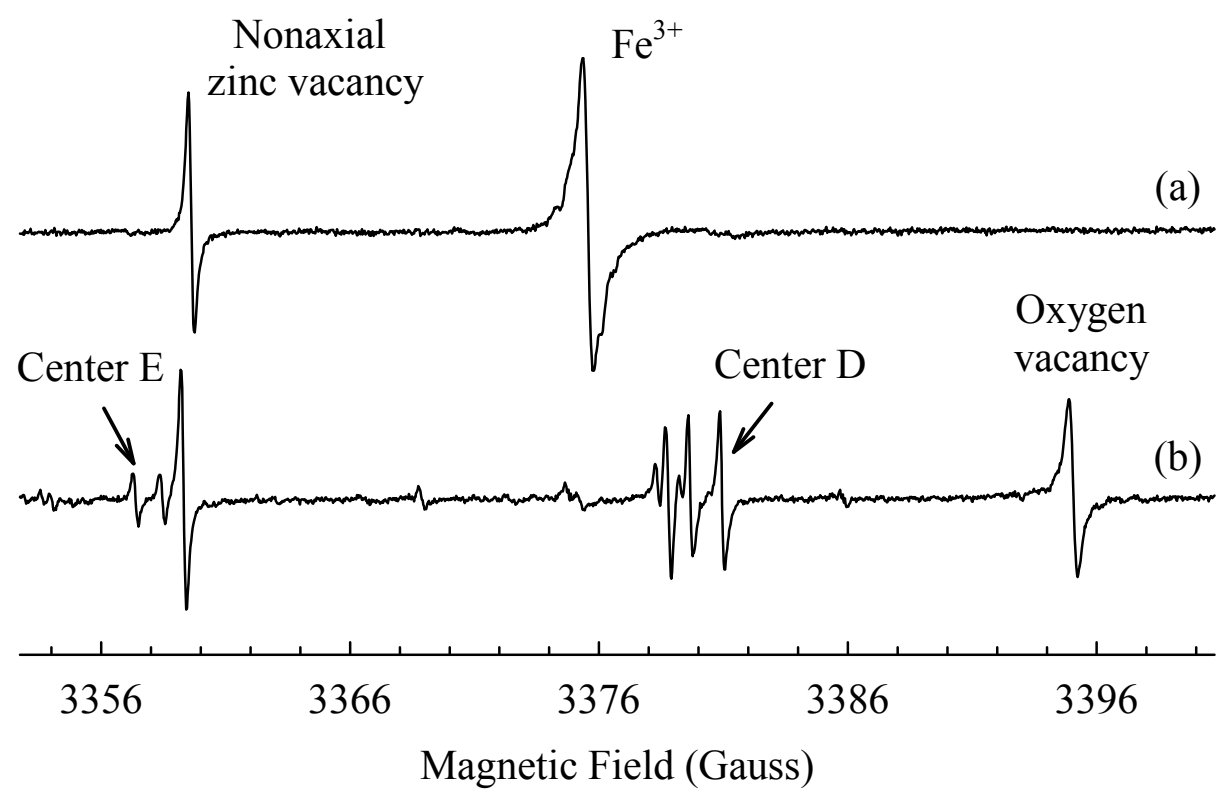

Figure 5.3. EPR spectra taken at $30 \mathrm{~K}$ from a $\mathrm{ZnO}$ crystal irradiated with $1.5 \mathrm{MeV}$ electrons. (a) Before illumination. (b) After a brief exposure to $325 \mathrm{~nm}$ laser light. 
between 3378.6 and 3380.9 G. Also, isolated axial singly ionized zinc vacancies, labeled Center B by Galland and Herve, ${ }^{22,94}$ appear near $3379 \mathrm{G}$ during the illumination. These latter five lines, representing four centers, in the center portion of Fig. 5.3(b) are shown in Fig. 5.4 with expanded horizontal and vertical scales. The $\mathrm{Fe}^{2+}$ ions formed in the irradiated crystal by the light are thermally stable only up to approximately $120 \mathrm{~K}$; they release the trapped electrons at this temperature ${ }^{78}$ and allow the oxygen and zinc vacancies to return to their pre-illumination charge states.

There are two doublets among these new acceptor-like EPR signals in Fig. 5.3(b). The doublet with lines at 3379.7 and $3380.9 \mathrm{G}$ is labeled Center D and the doublet with lines at 3357.6 and $3358.7 \mathrm{G}$ is labeled Center E. Hyperfine interactions with a nearby proton $(I=1 / 2)$ cause the observed 1.2 and $1.1 \mathrm{G}$ splittings. Direct evidence that the hyperfine splitting for Center $\mathrm{D}$ is due to a proton comes from the two forbidden

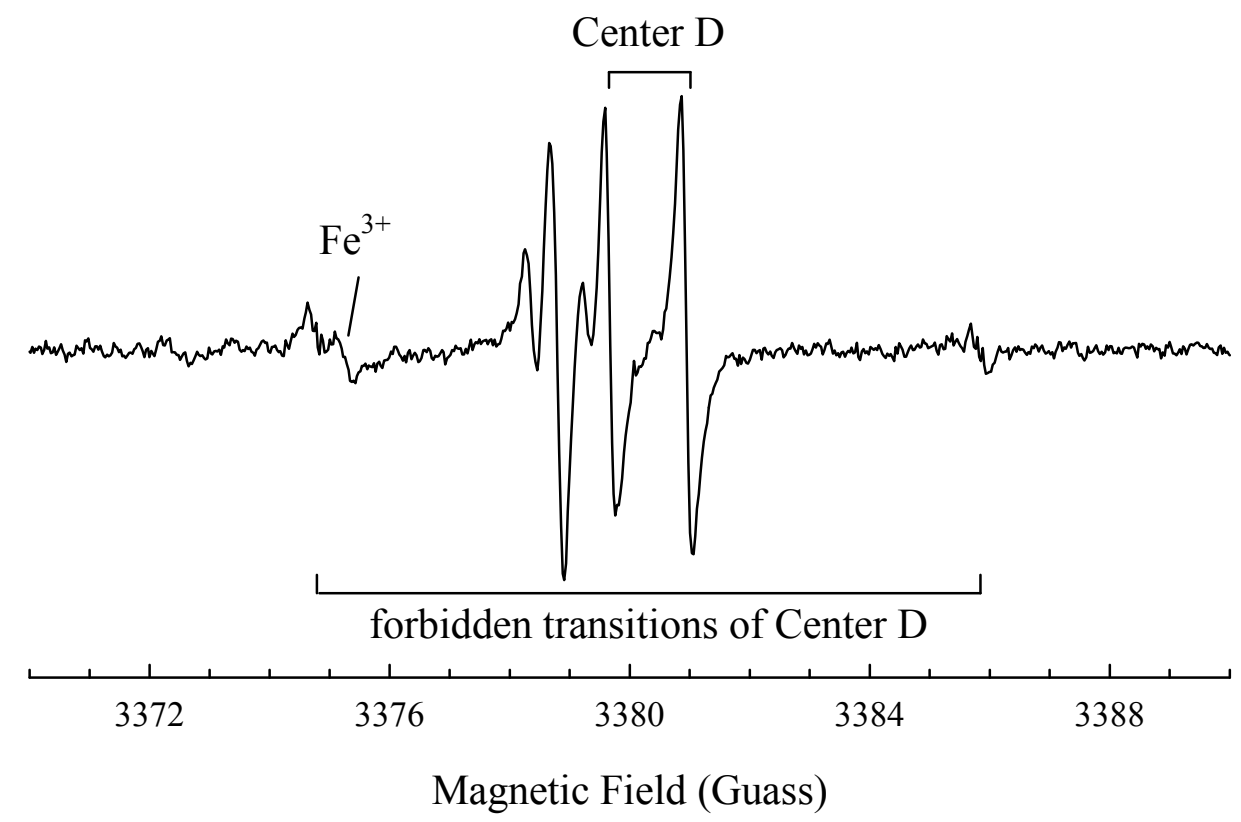

Figure 5.4. Expanded view of the center portion of the EPR spectrum in Fig. 5.3(b), showing four axial zinc-vacancy centers. The lower stick diagram illustrates forbidden transitions associated with Center D. A small signal from $\mathrm{Fe}^{3+}$ ions is also present. 
transitions observed at 3374.8 and $3385.8 \mathrm{G}$ (these lines are best seen in the expanded view in Fig. 5.4). These less intense lines are separated by $11.0 \mathrm{G}$ and are exactly centered on the two larger lines at 3379.7 and 3380.9 G, as indicated by the lower "stick" diagram in Fig. 5.4. This separation of the outer lines is very nearly twice the Larmor frequency of protons $(10.3 \mathrm{G}$ at $3380 \mathrm{G})$, and thus identifies the two outer lines as "forbidden" $\Delta \mathrm{m}_{\mathrm{S}}= \pm 1, \Delta \mathrm{m}_{\mathrm{I}}= \pm 1$ transitions of this $\mathrm{S}=1 / 2, \mathrm{I}=1 / 2$ spin system. ${ }^{108}$ The two larger, inner lines are the usual "allowed" $\Delta \mathrm{m}_{\mathrm{S}}= \pm 1, \Delta \mathrm{m}_{\mathrm{I}}=0$ transitions. The outer "forbidden" lines are observed in here because the hyperfine term and the nuclear Zeeman term in the spin Hamiltonian have similar magnitudes, and thus cause significant mixing of the four spin states.

Because the $c$-axis lines of Centers D and $\mathrm{E}$ are very near the isolated axial and the nonaxial singly ionized zinc vacancy spectra, respectively, Centers D and E are assigned to zinc vacancies with an $\mathrm{OH}^{-}$ion replacing one of the adjacent oxygen ions. The observed proton hyperfine splitting supports this model since its small value is consistent with a separation of approximately $3.2 \AA$ between the unpaired spin (i.e., the hole) and the proton. Center D has the unpaired spin localized on the axial oxygen neighbor with the $\mathrm{OH}^{-}$ion at one of the three neighboring nonaxial oxygen sites, while Center E has the unpaired spin localized on one of the three nonaxial oxygen neighbors with the $\mathrm{OH}^{-}$ion at one of the other two nonaxial oxygen sites or at the axial oxygen site. Very similar hydrogen-associated magnesium vacancy centers have been observed in $\mathrm{MgO}$, and their model has been clearly established using EPR and ENDOR. ${ }^{109}$ To simplify further discussion, the notation $\left(\mathrm{V}_{\mathrm{Zn}}^{-}-\mathrm{H}^{+}\right)^{0}$ will be used to describe the neutral paramagnetic forms of Centers $\mathrm{D}$ and $\mathrm{E}$. In this notation, the $\mathrm{V}_{\mathrm{Zn}}^{-}$represents both the zinc vacancy and the hole on one oxygen neighbor while the $\mathrm{H}^{+}$represents the extra positive charge provided by the $\mathrm{OH}^{-}$ion substituting for a separate oxygen $\left(\mathrm{O}^{2-}\right)$ neighbor. Another way of viewing Centers $\mathrm{D}$ and $\mathrm{E}$ is to envision a hydrogen atom $\left(\mathrm{H}^{0}\right)$ combining with a neutral zinc vacancy $\left(\mathrm{V}_{\mathrm{Zn}}^{0}\right)$. The electron from the hydrogen atom 
annihilates one of the two trapped holes at the neutral vacancy, thus leaving a single hole on one oxygen neighbor, while the proton from the hydrogen atom joins with a separate oxygen neighbor (an $\mathrm{O}^{2-}$ ion) to form the adjacent $\mathrm{OH}^{-}$ion.

A recent paper by Shi et al. ${ }^{110}$ describes "hidden" hydrogen in as-grown $\mathrm{ZnO}$ crystals (i.e., hydrogen that is not detected by infrared absorption techniques). They suggested that thermal anneals to $500{ }^{\circ} \mathrm{C}$ in a helium atmosphere dissociated a portion of the unseen $\mathrm{H}_{2}$ molecules initially present and formed observable $\mathrm{OH}^{-}$ions within the crystal. The results of Shi et al. ${ }^{110}$ provide a possible explanation for the source of the protons observed next to the zinc vacancies produced during the electron irradiation (i.e., $\mathrm{H}_{2}$ molecules dissociate during the electron irradiation and hydrogen atoms move to newly formed zinc vacancies). The neutral $\left(\mathrm{V}_{\mathrm{Zn}}^{-}-\mathrm{H}^{+}\right)^{0}$ centers and singly ionized $\left(\mathrm{V}_{\mathrm{Zn}}^{2-}-\mathrm{H}^{+}\right)^{-}$centers, specifically the $\mathrm{OH}^{-}$ions contained in them, are expected to be candidates for infrared absorption signals in the 3200 to $3700 \mathrm{~cm}^{-1}$ region. A preliminary investigation, however, at $5 \mathrm{~K}$ with light polarized $\mathrm{E} \perp \mathrm{c}$, did not reveal any observable $\mathrm{OH}^{-}$infrared spectra in the irradiated sample.

Three additional EPR signals related to zinc vacancies appear between 3378.6 and 3379.5 G during illumination, as shown in Fig. 5.3(b) and Fig. 5.4. One of these signals is the axial singly ionized zinc vacancy $\left(\mathrm{V}_{\mathrm{Zn}}^{-}\right)$. The other two signals are assigned to zinc vacancies having an unpaired spin on an axial oxygen neighbor with a next-nearest substitutional $\mathrm{Al}$ or $\mathrm{Ga}$ donor bonded to one of the three nonaxial oxygen ions. This places the $\mathrm{Al}$ or Ga ions sufficiently far away from the unpaired spin to make any weak hyperfine interactions with these nuclei unresolved in the EPR spectra. The earlier notation is followed and these acceptors are referred to as $\left(\mathrm{V}_{\mathrm{Zn}}^{-}-\mathrm{D}^{+}\right)^{0}$ centers, where $\mathrm{D}$ represents the Group III donor. Acceptor complexes of this type are commonly known as A centers and are often found in II-VI semiconductors. Their basic model is a cation vacancy with either a Group VII or a Group III donor impurity at the nearest or nextnearest site, respectively. ${ }^{111-113}$ Donor-bound excitons observed in photoluminescence 
spectra taken at $5 \mathrm{~K}$ show that $\mathrm{Al}$ and $\mathrm{Ga}$ donors are present in the electron-irradiated $\mathrm{ZnO}$ crystal, ${ }^{100}$ and thus support the assignment of two of the EPR lines between 3378.6 $\mathrm{G}$ and $3379.5 \mathrm{G}$ in Fig. 5.4 to $\left(\mathrm{V}_{\mathrm{Zn}_{\mathrm{n}}}^{-}-\mathrm{Al}^{+}\right)^{0}$ and $\left(\mathrm{V}_{\mathrm{Zn}_{\mathrm{n}}^{-}}^{-} \mathrm{Ga}^{+}\right)^{0}$ centers. For perspective, it should be noted that an analogous neutral center involving a magnesium vacancy, a trapped hole, and an $\mathrm{Al}^{3+}$ ion at a next-nearest neighbor site has been observed in $\mathrm{MgO}$ crystals with EPR and ENDOR. ${ }^{114-116}$ Because of their close proximity in magnetic field, it is difficult to specify at this time which of the three EPR lines between 3378.6 and 3379.5 G in Fig. 5.4 corresponds to zinc vacancies near Al, zinc vacancies near Ga, and isolated zinc vacancies.

Figure 5.5 shows the EPR spectrum of the neutral zinc vacancy $\left(\mathrm{V}_{\mathrm{Zn}}^{0}\right)$, labeled Center $\mathrm{C}$ by Galland and Herve. ${ }^{22}$ These data were taken at $30 \mathrm{~K}$ with the magnetic field parallel to the $c$ axis while using out-of-phase detection for the $100 \mathrm{kHz}$ modulation. The

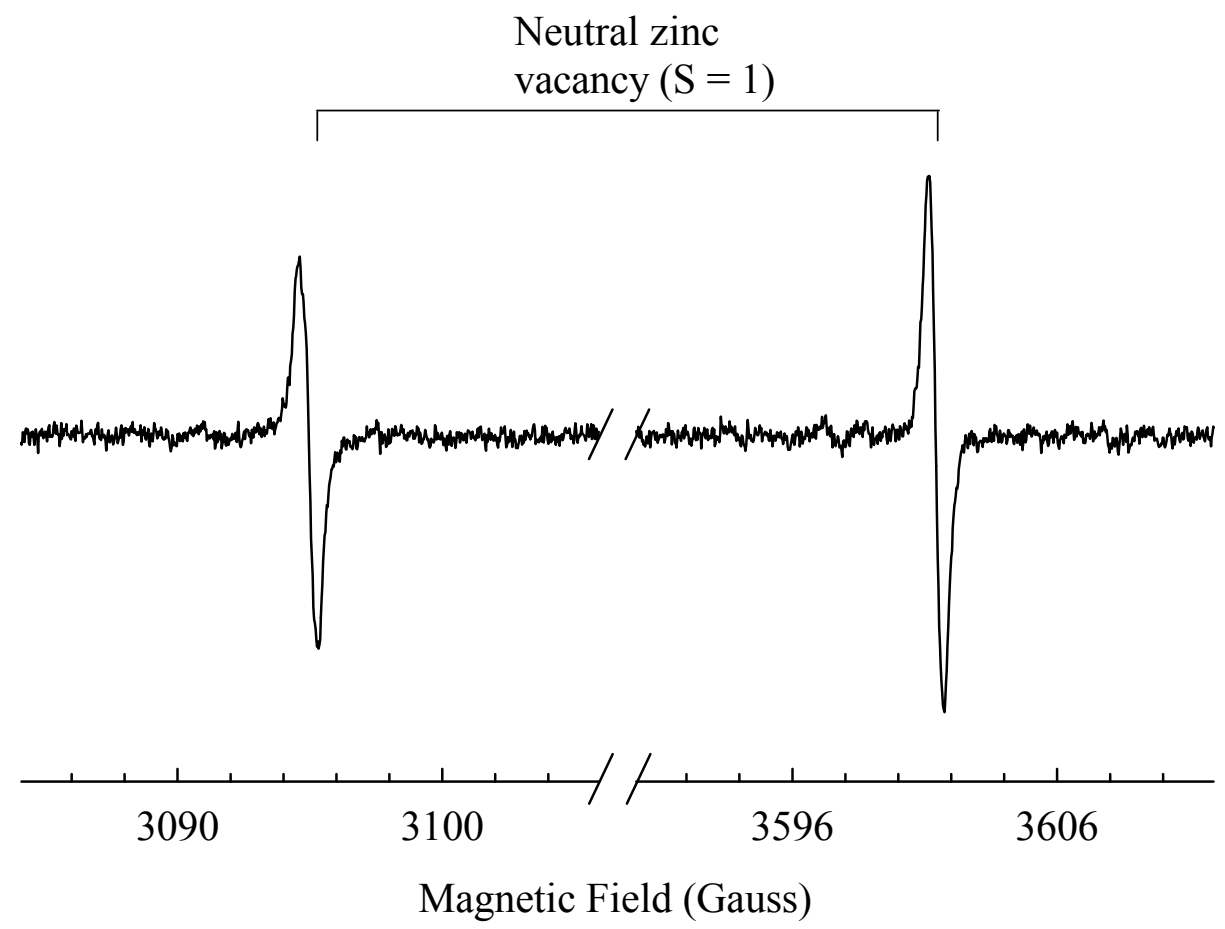

Figure 5.5. EPR spectrum of the neutral zinc vacancy $\left(\mathrm{V}_{\mathrm{Zn}}^{0}\right)$ in an electron-irradiated $\mathrm{ZnO}$ crystal. These data were taken at $30 \mathrm{~K}$ after a brief exposure to $325 \mathrm{~nm}$ laser light. 
isolated neutral zinc vacancy spectrum in Fig. 5.5 was produced at $30 \mathrm{~K}$ by a brief exposure to $325 \mathrm{~nm}$ laser light (this spectrum was not present in the electron-irradiated crystal prior to illumination at low temperature). The two holes at the zinc vacancy are separately trapped on two of the nonaxial oxygen neighbors, thus giving an $\mathrm{S}=1$ ground state. $^{22}$ The resulting two-line EPR spectrum has a $c$-axis separation of $506 \mathrm{G}$. From an analysis of the fine-structure splitting, Galland and Herve ${ }^{22}$ determined that the two holes were separated by approximately $3.75 \AA$. To simplify Fig. 5.5 , the center portion of the magnetic field sweep is not shown, as it is already presented in Fig. 5.3.

As can be seen in Figs. 5.3, 5.4, and 5.5, a variety of vacancy-associated defects are produced by irradiating a CVT-grown $\mathrm{ZnO}$ crystal with high-energy electrons. These results show the importance of using light at low temperature to induce changes in charge states. In most cases, the light converts initially unseen forms of these native defects into observable paramagnetic centers. In the case of isolated zinc vacancies, both the singly ionized and the neutral charge states are paramagnetic, and thus observable with EPR.

\subsubsection{Wavelength Dependence of Photoinduced Effects}

Figure 5.6 shows the relative response, at low temperature, of the electronirradiated crystal to different illumination wavelengths. Normalized changes in the EPR signals from $\mathrm{Fe}^{3+}$ ions, singly ionized oxygen vacancies, and axial zinc vacancies with an $\mathrm{OH}^{-}$ion at an adjacent oxygen site are illustrated. Although not shown, the neutral zinc vacancies $\left(\mathrm{V}_{\mathrm{Zn}}^{0}\right)$ behave the same as the $\left(\mathrm{V}_{\mathrm{Zn}}^{-}-\mathrm{H}^{+}\right)^{0}$ centers, i.e., the zinc vacancies with an adjacent $\mathrm{OH}^{-}$ion. This latter effect is expected since their EPR signals both represent neutral charge states and they most likely have similar ground state energies within the band gap. The data in Fig. 5.6 were obtained using the lamp/monochromator combination as the light source. The sample was held at room temperature for several minutes, cooled in the dark to $30 \mathrm{~K}$, and then exposed to light at the specified wavelength. The EPR spectra were recorded at $30 \mathrm{~K}$ after waiting approximately $5 \mathrm{~min}$ for the light to 
establish a new steady state distribution of charge. This procedure was repeated at each successive wavelength, including warming to room temperature and cooling in the dark. In order to maintain a constant photon flux on the microwave cavity, the light intensity was adjusted for each wavelength using a variable neutral density filter. At $400 \mathrm{~nm}$, the incident power on the microwave cavity was $0.93 \mathrm{~mW}$. The bandpass of the monochromator changed from 8.5 to $19 \mathrm{~nm}$ over the wavelength range 350 to $750 \mathrm{~nm}$.

Threshold wavelengths are difficult to determine precisely in Fig. 5.6, so only approximate wavelengths are specified where significant changes in defect behavior are first observed. As seen in Fig. 5.6, wavelengths shorter than $600 \mathrm{~nm}$ destroy the $\mathrm{Fe}^{3+}$ ions and produce singly ionized oxygen vacancies $\left(\mathrm{V}_{\mathrm{O}}^{+}\right)$. This suggests that the

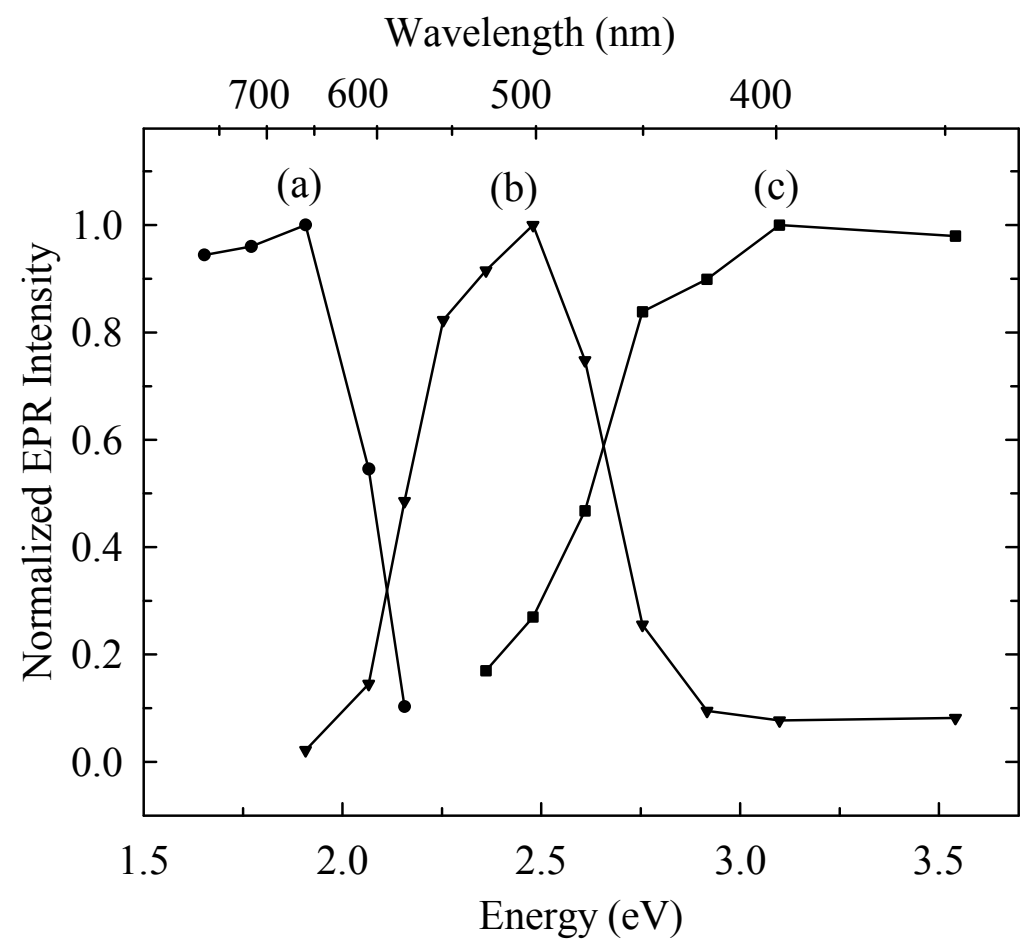

Figure 5.6. Wavelength dependence of changes in charge state while illuminating at $30 \mathrm{~K}$. The monitored EPR signals are (a) $\mathrm{Fe}^{3+}$ ions, (b) singly ionized oxygen vacancies $\left(\mathrm{V}_{\mathrm{O}}^{+}\right)$, and (c) zinc vacancies with an $\mathrm{OH}^{-}$ ion at an adjacent oxygen site, i.e., $\left(\mathrm{V}_{\mathrm{Zn}}^{-}-\mathrm{H}^{+}\right)^{0}$ centers. 
wavelength region near $2.1 \mathrm{eV}$ is the threshold for pumping an electron from a neutral oxygen vacancy to the conduction band. $\mathrm{An} \mathrm{Fe}^{3+}$ ion then traps the free electron and forms an $\mathrm{Fe}^{2+}$ ion. These $\mathrm{Fe}^{2+}$ ions are thermally unstable above approximately $120 \mathrm{~K}$, and heating to this temperature after exposure to light restores the pre-illumination condition (i.e., the $\mathrm{Fe}^{3+}$ signal returns and the singly ionized oxygen-vacancy signal disappears). A low-temperature band gap of approximately $3.4 \mathrm{eV}$ combined with the present observation of a threshold near $2.1 \mathrm{eV}$ for light to produce singly ionized oxygen vacancies places the ground state of the neutral oxygen vacancy deep within the gap, approximately $1.3 \mathrm{eV}$ from the top of the valence band. The two sets of data in Fig. 5.6 representing the destruction of the $\mathrm{Fe}^{3+}$ ions and the production of the $\mathrm{V}_{\mathrm{O}}^{+}$centers do not cross exactly at their mid-intensity positions. This is attributed to errors associated with alignment of the magnetic field parallel to the $c$ axis of the crystal. In particular, the intensity of the $\mathrm{Fe}^{3+}$ signal is sensitive to this alignment (a misalignment of $0.5^{\circ}$ from the $c$ direction corresponds to a decrease of approximately $8 \%$ in signal intensity).

For illumination wavelengths shorter than $500 \mathrm{~nm}$, an additional excitation path emerges in Fig. 5.6. These higher-energy photons pump electrons to the conduction band from zinc vacancies with an $\mathrm{OH}^{-}$ion at an adjacent oxygen site and also from isolated singly ionized zinc vacancies. This threshold near $2.5 \mathrm{eV}$ to form $\left(\mathrm{V}_{\mathrm{Zn}}^{-}-\mathrm{H}^{+}\right)^{0}$ and $\mathrm{V}_{\mathrm{Zn}}^{0}$ defects places the ground states of the $\left(\mathrm{V}_{\mathrm{Zn}}^{2-}-\mathrm{H}^{+}\right)^{-}$and $\mathrm{V}_{\mathrm{Zn}}^{-}$acceptors approximately 0.9 $\mathrm{eV}$ above the valence band.

The light-induced formation of singly ionized oxygen vacancies, when the electron-irradiated crystal is at low temperature, appears to have a "peak" in Fig. 5.6 at $500 \mathrm{~nm}$. Earlier investigators ${ }^{91,117,118}$ also observed a peak near this wavelength in the excitation spectrum of singly ionized oxygen vacancies. The peak in the production of the $\mathrm{V}_{\mathrm{O}}^{+}$centers in Fig. 5.6, however, does not represent a "resonance" (i.e., a discrete transition from one state to another) since one process is present for wavelengths longer than $500 \mathrm{~nm}$ and competing processes are present for shorter wavelengths. For 
wavelengths longer than $500 \mathrm{~nm}$, the process is relatively simple. Electrons are trapped on the $\mathrm{Fe}^{3+}$ ions, thus forming $\mathrm{Fe}^{2+}$ ions, and oxygen vacancies are converted from neutral $\left(\mathrm{V}_{\mathrm{O}}^{0}\right)$ to singly ionized $\left(\mathrm{V}_{\mathrm{O}}^{+}\right)$states. For wavelengths shorter than $500 \mathrm{~nm}$, the situation is more complex as zinc vacancy defects are now involved and different steadystate conditions are established. For these shorter wavelengths, electrons are pumped to the conduction band from both oxygen and zinc vacancies. There are not enough $\mathrm{Fe}^{3+}$ ions (and other shallow donors) in the crystal to trap all of these electrons being pumped to the conduction band, and thus some electrons quickly return to the oxygen and zinc vacancies. The relative electron trapping cross-sections of the different vacancy centers then becomes a critical factor in determining the resulting steady-state distribution of the various charge states under illumination. The $\mathrm{V}_{\mathrm{O}}^{+}$centers with an effective positive charge have a larger electron trapping cross-section than the neutral $\left(\mathrm{V}_{\mathrm{Zn}}^{-}-\mathrm{H}^{+}\right)^{0}$ and $\mathrm{V}_{\mathrm{Zn}}^{0}$ centers, and the photoinduced electrons preferentially return to the oxygen vacancies. The data in Fig. 5.6 were taken after the illumination produced a steady state for each wavelength, thus the intensity of the singly ionized oxygen vacancy signal approaches zero at the shorter wavelengths and the signal due to the $\left(\mathrm{V}_{\mathrm{Zn}}^{-}-\mathrm{H}^{+}\right)^{0}$ centers is maximized at these wavelengths. In other words, a redistribution of electrons from zinc vacancy centers to oxygen vacancies occurs during longer exposures to short wavelength light.

Figure 5.7 contains time-dependence data that better illustrate the competing processes taking place during exposure to short wavelengths of light. These data, obtained while illuminating the electron-irradiated crystal with $325 \mathrm{~nm}$ laser light at 30 $\mathrm{K}$, show the time evolution of the EPR signals from $\mathrm{Fe}^{3+}$ ions, singly ionized oxygen vacancies $\left(\mathrm{V}_{\mathrm{O}}^{+}\right)$, and zinc vacancies with an adjacent $\mathrm{OH}^{-}$ion, i.e., $\left(\mathrm{V}_{\mathrm{Zn}}^{-}-\mathrm{H}^{+}\right)^{0}$ centers. The data for each defect were taken by placing the magnetic field at the position corresponding to the maximum of that defect's EPR derivative signal and then monitoring the intensity of the EPR signal as a function of time after turning on the laser. 


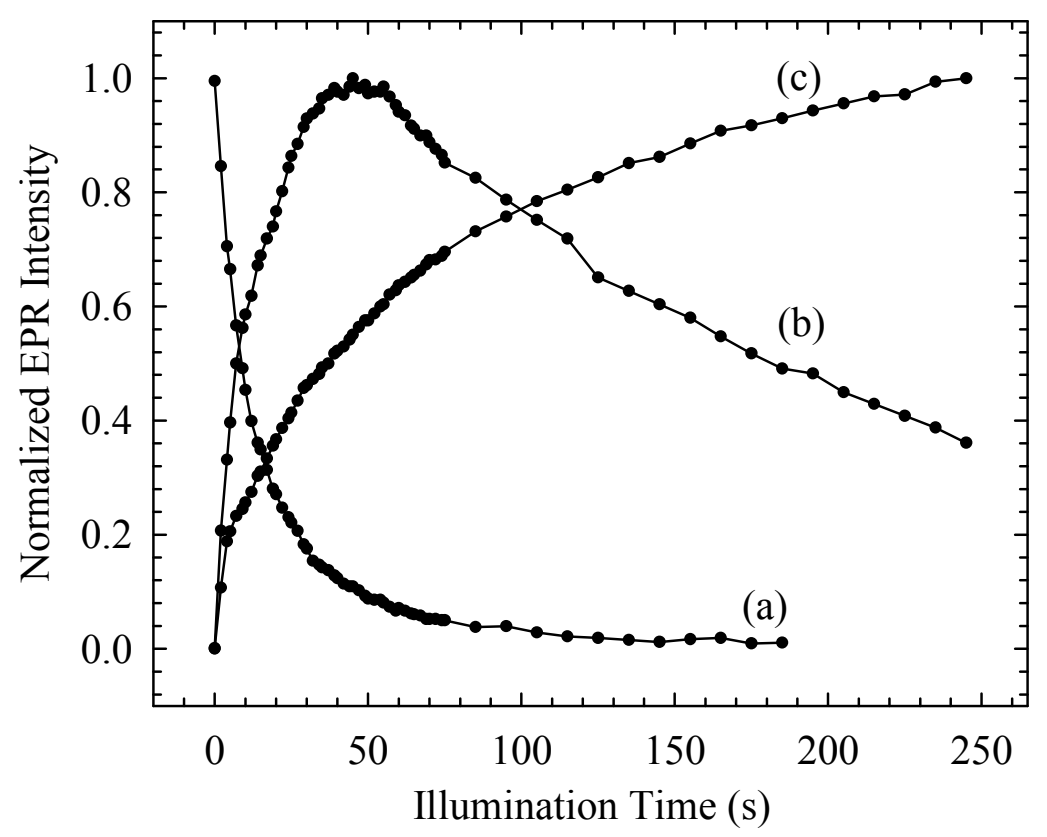

Figure 5.7. Time dependence of changes in charge state while illuminating at $30 \mathrm{~K}$ with $325 \mathrm{~nm}$ laser light. The monitored EPR signals are (a) $\mathrm{Fe}^{3+}$ ions, (b) singly ionized oxygen vacancies ( $\mathrm{V}_{\mathrm{O}}^{+}$), and (c) zinc vacancies with an $\mathrm{OH}^{-}$ion at an adjacent oxygen site, i.e., $\left(\mathrm{V}_{\mathrm{Zn}}^{-}-\mathrm{H}^{+}\right)^{0}$ centers.

Before going to the next EPR signal, the sample was "reset" by warming briefly to room temperature. Normalized changes in the EPR intensities are plotted in Fig. 5.7 for the first 4 min of illumination.

As can be seen in Fig. 5.7, the $325 \mathrm{~nm}$ light quickly produces the $\mathrm{V}_{\mathrm{O}}^{+}$signal and destroys the $\mathrm{Fe}^{3+}$ signal as electrons are pumped from the neutral oxygen vacancies to the conduction band. The $\mathrm{V}_{\mathrm{O}}^{+}$signal reaches a maximum when there are no more $\mathrm{Fe}^{3+}$ electron traps. With continuing illumination, the $\mathrm{V}_{\mathrm{O}}^{+}$signal slowly decreases, and the $\left(\mathrm{V}_{\mathrm{Zn}}^{-}-\mathrm{H}^{+}\right)^{0}$ signal steadily grows. This latter behavior observed at longer times (i.e., greater than 1 min in Fig. 5.7) describes the preferential trapping of photoexcited electrons at singly ionized oxygen vacancies, instead of at neutral $\left(\mathrm{V}_{\mathrm{Zn}}^{-}-\mathrm{H}^{+}\right)^{0}$ centers. For times longer than the 4 min (not shown in Fig. 5.7), the EPR signal from the 
$\left(\mathrm{V}_{\mathrm{Zn}}^{-}-\mathrm{H}^{+}\right)^{0}$ centers continues to grow at a diminishing rate and the EPR signal from the $\mathrm{V}_{\mathrm{O}}^{+}$centers continues to decrease at a similar rate until equilibrium is reached for both centers. The initial rapid growth of the $\mathrm{V}_{\mathrm{O}}^{+}$signal in Fig. 5.7 suggests that the probability of pumping an electron from a $\mathrm{V}_{\mathrm{O}}^{0}$ center is greater than pumping an electron from a $\left(\mathrm{V}_{\mathrm{Zn}}^{2-}-\mathrm{H}^{+}\right)^{-}$or $\mathrm{V}_{\mathrm{Zn}}^{-}$center.

\subsection{Discussion}

\subsubsection{Present Results}

Oxygen vacancies and zinc vacancies were produced in a vapor-transport-grown $\mathrm{ZnO}$ crystal by electron irradiation and then studied at low temperature using the EPR technique. The electron irradiation produced a large concentration of acceptors ( zinc vacancies), thus significantly lowering the Fermi level. This allows the $\mathrm{Fe}^{2+}$ to convert to the $\mathrm{Fe}^{3+}$ state and the EPR signals from $\mathrm{Fe}^{3+}$ ions and nonaxial singly ionized zinc vacancies to be observed in the irradiated crystal before illumination at low temperature. During and after illumination at low temperature, EPR signals were observed from singly ionized oxygen vacancies, isolated axial singly ionized zinc vacancies, isolated neutral zinc vacancies, zinc vacancies having an $\mathrm{OH}^{-}$ion at an adjacent oxygen site, and zinc vacancies having an $\mathrm{Al}$ or Ga donor at the nearest cation site. A lamp and monochromator were used to investigate photoinduced changes in charge states of these vacancy centers and the $\mathrm{Fe}^{3+}$ ions. The photon energy thresholds observed when varying the illumination wavelength suggest that the ground states of the neutral oxygen vacancy and the singly ionized zinc vacancy are approximately 1.3 and $0.9 \mathrm{eV}$ above the valence band, respectively.

A complex wavelength dependence for producing singly ionized oxygen vacancies $\left(\mathrm{V}_{\mathrm{O}}^{+}\right)$with light was observed and explained. The apparent "peak" near 500 $\mathrm{nm}$ in the photoexcitation production curve is not due to a discrete absorption resonance, but is a result of the emergence of competing processes involving additional defects in 
the crystal. This is demonstrated by the change of the EPR signals as a function of illumination time, showing that over a short period of time the EPR signal from the $\mathrm{V}_{\mathrm{O}}^{+}$ grows to a relatively intense signal before reducing to a much smaller intensity at longer times. One excitation mechanism is active for wavelengths longer than $500 \mathrm{~nm}$, while two or more mechanisms are active for wavelengths shorter than $500 \mathrm{~nm}$. At these shorter wavelengths, a competition among hole trapping sites occurs.

The presence of impurity donors played an important role in the present observation of paramagnetic states of the oxygen and zinc vacancies in $\mathrm{ZnO}$. In the CVT-grown crystal used in the present study, the dominant donor defects before irradiation were Group III impurities and $\mathrm{Fe}^{2+}$ ions (with ionization energies of approximately 65 and $240 \mathrm{meV}$, respectively). After irradiation, the crystal was much less $\mathrm{n}$ type and these shallow donors were nearly all in their singly ionized charge states. The $\mathrm{Fe}^{3+}$ donors, present after the irradiation, permitted stable trapping of electrons that were photoinduced into the conduction band. It is possible that other crystals grown by the CVT method as well as crystals grown by other techniques (e.g., hydrothermally or high-temperature melt) may have a different "mix" of donors and acceptors (i.e., different types and different relative concentrations), and thus the observed distribution of charge states of vacancies in the dark and under illumination may be correspondingly different.

\subsubsection{Comparison with previous studies}

This work presented in this chapter has shown the complex nature of the correlation of multiple defects in one sample. A previously unreported EPR signal has been identified as a complex of a zinc vacancy with hydrogen attached to a neighboring oxygen. Other zinc vacancy-donor complexes have also been observed with aluminum, gallium and indium being the most likely sources. The ground state energies of these defects and of the neutral oxygen vacancy have been measured in this work as well. It is easy to overlook the differences between this study and previous studies. The importance 
of the presence of certain defects in this sample was mentioned above. Notably, the presence of iron in the sample played one of the most important roles in this study. The $\mathrm{Fe}^{3+}$ may not have been the focus of this study, but without it observation of the any signals from the oxygen vacancy or the zinc vacancy complexes may have proven difficult if not impossible. The presence of compensating acceptor states is also important to lower the Fermi level to convert the $\mathrm{Fe}^{2+}$ to $\mathrm{Fe}^{3+}$. In this sample, isolated zinc vacancies and zinc vacancy complexes provided this compensation.

In some of the previous works, however, there are either different defects present in the material or there simply is no discussion of the presence of other defects. The current study showed certain differences in EPR responses than in some of the previous studies of oxygen and zinc vacancies in $\mathrm{ZnO}$. Smith and Vehse reported EPR results of electron-irradiated $\mathrm{ZnO}$ and identified the singly ionized oxygen vacancy. ${ }^{25}$ Their study showed three primary EPR signals which were observable in the dark at room temperature. Upon illumination with white light, the singly ionized oxygen vacancy signal greatly increased in intensity. The response of the other two signals is not discussed, but their data showed that the signals are easily observable. Their results differ somewhat with respect to the results from the current study. The sample used in the current work showed no sign of the singly ionized oxygen vacancy EPR signal at room temperature in the dark. The signal is either not present or too small to be observable over the noise. Under illumination at room temperature, the singly ionized oxygen vacancy appears with an low signal to noise ratio. Low temperatures and illumination of wavelengths shorter than $500 \mathrm{~nm}$ were required to observe the singly ionized oxygen vacancy EPR signal. In his Ph.D. dissertation, ${ }^{119}$ Smith stated that the singly ionized oxygen vacancy did not show any response to uv-illumination, raising the question of whether there was no response, the response was not seen because of a similar growth/decay process seen in the current work, or some other possible explanation. The intensity of the oxygen vacancy signal is of particular interest. The 
ability to see the oxygen vacancy EPR signal before illumination could simply be due to the overall intensity of the signal compared to the EPR signal from the current study. Many questions remain about the actual concentration of the oxygen vacancies and the effect of the unidentified signals present in the EPR spectrum. Later studies show an EPR signal due to $\mathrm{Fe}^{3+}$ in the same region as the unidentified "central" line. The angular dependence of this signal, however, does not match that of the $\mathrm{Fe}^{3+}$.

In the current study, compensating acceptors (zinc vacancies) and an electron trap $\left(\mathrm{Fe}^{3+}\right)$ were necessary to observe the EPR signal due to the singly ionized oxygen vacancy. Smith and Vehse ${ }^{25}$ suggested that the light sensitivity is due to the removal of an electron by the light, but do not discuss where that electron may be trapped. There also is no discussion on the other defects shown in their data or how they may interact with oxygen vacancies.

In the work by Taylor et al. ${ }^{34}$ in 1970 , EPR signals from the singly ionized oxygen vacancy and the non-axial singly ionized zinc vacancy were observed in neutronirradiated zinc oxide grown by the vapor phase method. In their study, many signals were observed in the region of $g=2.0018$ to $g=2.056$, one set of which was the EPR spectrum due to the non-axial singly ionized zinc vacancy. They did not, however, observe any EPR signal from the axial singly ionized zinc vacancy in that region. Another difference of their results from the results in the current work is the lack of illumination to produce these EPR signals. Although Taylor $e t . A l^{34}$ specifically state that u.v. illumination was not necessary to produce these signals, they do not specify whether or not illumination affects the signals at all. They suggest that the sample's radioactivity from the neutron irradiation as the source of the excitation for the defect centers. This could be an explanation for the presence of the paramagnetic centers without any illumination.

Around the same time as the study by Taylor et al., Galland and Herve ${ }^{22}$ were also studying zinc vacancies in zinc oxide. They used electron irradiation at room 
temperature and observed the singly ionized oxygen vacancy along with at least a few other centers. Their results focused only on the centers related to the zinc vacancy, including the axial and non-axial singly ionized zinc vacancies and the neutral zinc vacancy. In their study, the EPR signals related to the zinc vacancy were observable at $90 \mathrm{~K}$ without any illumination, which is different than the results in the current work. Galland and Herve observed a large increase in intensity of these signals using uv illumination. There is no discussion of the other centers observed in their sample, nor is there any discussion on any other effects of illumination.

Later in 1972 , Locker and Meese ${ }^{90,91}$ were studying the displacement threshold energies for producing zinc vacancies and oxygen vacancies in $\mathrm{ZnO}$ with high-energy electron irradiation. In this study they produced oxygen vacancies using a lower electron energy than had been done before. This was done by a multistep process by first irradiating with $900 \mathrm{keV}$ to produce both oxygen vacancies and zinc vacancies. Then they anneal the sample to $300{ }^{\circ} \mathrm{C}$ to remove the oxygen vacancies. Following a second electron irradiation with energy below $600 \mathrm{keV}$, the EPR signal of the oxygen vacancy was observed. Locker and Meese explained that the oxygen vacancies are present if irradiated with energies below $600 \mathrm{keV}$, but not observable. The production of the zinc vacancies with irradiation energy above $600 \mathrm{keV}$ lowers the Fermi level and allows the oxygen vacancies to be in an observable paramagnetic state. In this study, Locker and Meese also observed EPR signals from $\mathrm{Fe}^{3+}$ and $\mathrm{Mn}^{2+}$ that increased after irradiation. In the current study the $\mathrm{Fe}^{3+}$ signal was present after the electron irradiation, but was not present before irradiation as with the study by Locker and Meese. Another significant result in their study was a wavelength dependence at room temperature of the production of the EPR signal like was performed in the current study at low temperature. Their wavelength dependence showed a rather broad band centered around $530 \mathrm{~nm}(530 \mathrm{~nm}$ excitation produced the signal with the greatest intensity). Locker and Meese associated this band to an absorption band that is commonly present in electron-irradiated zinc 
oxide. With the results in the current study the excitation spectrum at room temperature would be expected to be different. For a simple case, instead of a band, the shorter wavelength side of the band would be expected to remain constant after the peak. A more complex process may be involved, however, and a future study could prove useful in providing more detail on the room temperature excitation.

In 1976, a study by Soriano and Galland ${ }^{27}$ focused on the effect of illumination of the EPR signal due to oxygen vacancy in $\mathrm{ZnO}$. In their study, samples of Lithium-doped $\mathrm{ZnO}$ was first annealed to $800^{\circ} \mathrm{C}$, and then irradiated at room temperature with highenergy electrons of $0.6,0.8$, and $3.0 \mathrm{MeV}$ energy. Like with the sample in the current study, a yellow color change was observed in the samples. Soriano and Galland were able to see the oxygen vacancy EPR signal at room temperature only after illumination. Once the illumination was removed, the EPR signal would completely decay away. At $77 \mathrm{~K}$, the photosensitivity was studied in more detail using different wavelengths of light. First, the sample was illuminated by visible light to produce the oxygen vacancy EPR signal. At this temperature, the signal is stable once the light is removed (behavior which is consistent with the sample used in the current work). In the next step, the sample was illuminated with uv light and the EPR signal was observed to reduce to $20 \%$ of the intensity before the uv illumination. They also noted that illumination with light of energy greater than the bandgap would reduce the EPR signal to as low as $4 \%$ of its intensity before uv illumination. These results could agree with the results of the current work, however, in the next step of their study Soriano and Galland observed that if the sample was once again illuminated with visible light that the EPR signal is restored. If these steps are taken with the sample in the current work, the results are similar. Once the oxygen vacancy signal is produced, uv illumination causes the EPR signal to quickly decay, but the signal slowly recovers when illuminated once again by visible light. Similarly, if the sample is illuminated with $650 \mathrm{~nm}$ light after the signal has been produced, the EPR signal from the oxygen vacancy will slowly decay. This would be a 
different process. The likely explanation is that $650 \mathrm{~nm}$ light is too low of energy to pump electrons off the zinc vacancies or oxygen vacancies, but large enough energy to pump the trapped electrons off of the $\mathrm{Fe}^{2+}$ into the conduction band to be retrapped at the oxygen vacancy, converting each back to their pre-illumination charge states.

The discussion of the previous studies in this section has shown that different samples can have some effect on how the EPR signals may behave under different illumination as well as showing how the current work has produced useful information that compliments the information yielded from the previous studies that have been done on zinc and oxygen vacancies in $\mathrm{ZnO}$. These different behaviors can be explained by the presence of different defects in varying concentrations. The current work demonstrated the complex nature of how electrons and holes are excited and trapped to produce zinc and oxygen vacancies at low temperatures. Future studies focusing on how the presence of other types of acceptors and donors affect zinc and oxygen vacancies could provide answers to some of the questions about the different behavior in different samples, and thus a better understanding of the defects in $\mathrm{ZnO}$ and of the affect they have on the properties of $\mathrm{ZnO}$. 


\section{CHAPTER 6 \\ Nitrogen Acceptors in Zinc Oxide}

\subsection{Introduction}

This chapter presents the results of an EPR study of the hyperfine associated with the nitrogen acceptor in $\mathrm{ZnO}$. Previous studies have described EPR spectra associated with isolated substitutional nitrogen $\left(\mathrm{N}^{0}\right)$ acceptors ${ }^{120,121}$ and from molecular nitrogen $\left(\mathrm{N}_{2}^{-}\right)$acceptors. ${ }^{122}$ The results presented in the following sections further characterize the hyperfine interactions with the nitrogen nucleus and the nearest-neighbor zinc nuclei for the isolated nitrogen acceptor. The angular dependence data obtained from EPR was analyzed by complete diagonalization of the full spin Hamiltonian. Nuclear electric quadrupole effects are included in the nitrogen analysis, thus yielding a value for the nuclear quadrupole and more accurate $g$ values and nitrogen hyperfine parameters. The hyperfine parameters for the axial and non-axial zinc neighbors were also obtained.

The bulk $\mathrm{ZnO}$ sample used in the present study was cut from a larger boule grown at Eagle-Picher (Miami, OK). This sample was grown using a seeded chemical vapor transport method, with $\mathrm{N}_{2}$ added to the gas stream to serve as the doping source. As grown, this boule has a distinct yellow discoloration from the normal clear crystals. Room-temperature Hall measurements showed that this as-grown material was n type, with a free-carrier concentration value of $2 \times 10^{16} \mathrm{~cm}^{-3}$. This value is approximately a factor of five less than the values obtained for the usual undoped clear $\mathrm{ZnO}$ crystals grown at Eagle-Picher. The sample used in this EPR experiment had approximate dimensions of $5 \times 3 \times 2 \mathrm{~mm}^{3}$. All of the EPR data were obtained using the Bruker EMX spectrometer operating near $9.47 \mathrm{GHz}$. Values of the static magnetic field were obtained with a proton gaussmeter. A small $\mathrm{Cr}$-doped $\mathrm{MgO}$ crystal was used to correct for the difference in magnetic field between the sample and the probe tip of the gaussmeter (the

isotropic $\mathrm{g}$ value for $\mathrm{Cr}^{3+}$ in $\mathrm{MgO}$ is 1.9800). The sample temperature was maintained 
using an Oxford Instruments helium-gas flow. A cw helium-cadmium laser (operating at $442 \mathrm{~nm}$ ) was used to produce the isolated nitrogen EPR spectra.

\subsection{Experimental Results}

\subsubsection{Nitrogen Hyperfine}

Figure 6.1(a) shows the three line EPR spectrum of the isolated neutral nitrogen acceptor previously reported by Carlos et al. ${ }^{120}$ and Garces et al. ${ }^{121}$ This spectrum was obtained with the magnetic field along the $c$ axis of the crystal while the sample was illuminated with $442 \mathrm{~nm}$ laser light at $6 \mathrm{~K}$. The three lines in the spectrum indicate and interaction with a single ${ }^{14} \mathrm{~N}$ nucleus ( $\mathrm{I}=1,99.6 \%$ abundant $)$. The isolated nitrogen spectrum has axial symmetry about the $c$ axis and thus shifts, but does not split, as the

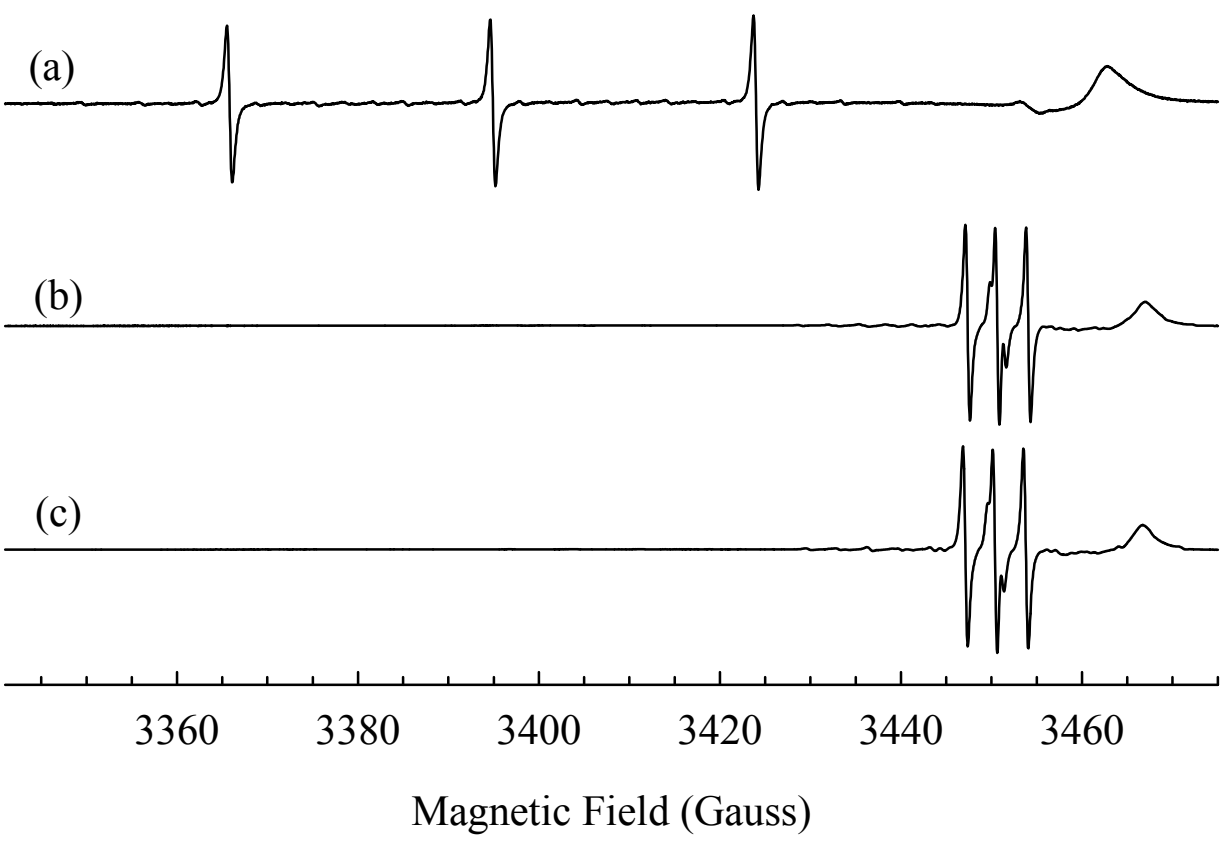

Figure 6.1. EPR spectra of the isolated neutral nitrogen acceptor taken at $5 \mathrm{~K}$ during illumination with $442 \mathrm{~nm}$ laser light. The magnetic field (a) along the $c$ axis, (b) perpendicular to the $c$ axis along the high symmetry direction labeled "hs 1", and (c) perpendicular to the $c$ axis along the high symmetry direction labeled "hs2". 
magnetic field is rotated from parallel to the $c$ axis to perpendicular to the $c$ axis. Figures 6.1(b) and 6.1(c) show the neutral nitrogen acceptor EPR signal with the magnetic field perpendicular to the $c$ axis. The magnetic fields in these two traces are along two highsymmetry axes that lie perpendicular to the $c$ axis and are $30^{\circ}$ from one another.

As the magnetic field is rotated away from the $c$ axis, the nitrogen hyperfine EPR signals associated with "forbidden" transitions become more intense due to mixing of spin states. The "forbidden" EPR signals become observable when the direction of the magnetic field is more than $60^{\circ}$ from the $c$ axis. Near the basal plane, the "forbidden" EPR signals can have intensities comparable to the "allowed" EPR transitions. In Figs. 6.1(b) and 6.1(c), EPR signals from two of these "forbidden" transitions can be observed on either side of the middle of the three "allowed" EPR signals. Figure 6.2 shows the angular dependence of the "allowed" and the "forbidden" transitions (solid and dashed lines respectively) of the neutral nitrogen acceptor as the magnetic field is rotated away from the $c$ axis toward the basal plane. The discrete points in Fig. 6.2 represent experimental data. These data were fit using the following spin Hamiltonian.

$$
\hat{H}=\beta \mathbf{B} \cdot \overrightarrow{\mathbf{g}} \cdot \hat{\mathbf{S}}+\hat{\mathbf{I}} \cdot \overrightarrow{\mathbf{A}} \cdot \hat{\mathbf{S}}+\hat{\mathbf{I}} \cdot \overrightarrow{\mathbf{Q}} \cdot \hat{\mathbf{I}}-\mathrm{g}_{\mathrm{N}} \beta_{\mathrm{N}} \mathbf{B} \cdot \hat{\mathbf{I}}
$$

Only five parameters $\left(\mathrm{g}_{\|}, \mathrm{g}_{\perp}, \mathrm{A}_{\|}, \mathrm{A}_{\perp}\right.$, and $\left.\mathrm{P}\right)$ are needed to describe this axial system. The $\mathrm{Q}$ matrix is traceless with $\mathrm{Q}_{\mathrm{xx}}=-\mathrm{P}, \mathrm{Q}_{\mathrm{yy}}=-\mathrm{P}$, and $\mathrm{Q}_{\mathrm{zz}}=2 \mathrm{P}$. The parameter $\mathrm{P}$ is defined as e $2 \mathrm{qQ} /[4 \mathrm{I}(2 \mathrm{I}-1)]$, where eq is the electric field gradient and $\mathrm{Q}$ is the nuclear quadrupole moment. Fitting to the experimental data in Fig. 6.2 gave $\mathrm{g}_{\|}=1.9949, \mathrm{~g}_{\perp}=$ 1.9631, $\mathrm{A}_{\|}=81.26 \mathrm{MHz}, \mathrm{A}_{\perp}=7.95 \mathrm{MHz}$, and $\mathrm{P}=-1.475 \mathrm{MHz}$. The present analysis consisted of a full diagonalization of the spin Hamiltonian, including quadrupole effects. Although not large, these second-order nuclear quadrupole effects are easily measured for the nitrogen acceptor, especially in the spectrum with the magnetic field oriented in and near the basal plane. These g values and hyperfine values are more accurate than those obtained in previous studies. ${ }^{120,121}$ The solid and dashed lines in Fig. 6.2 represent 


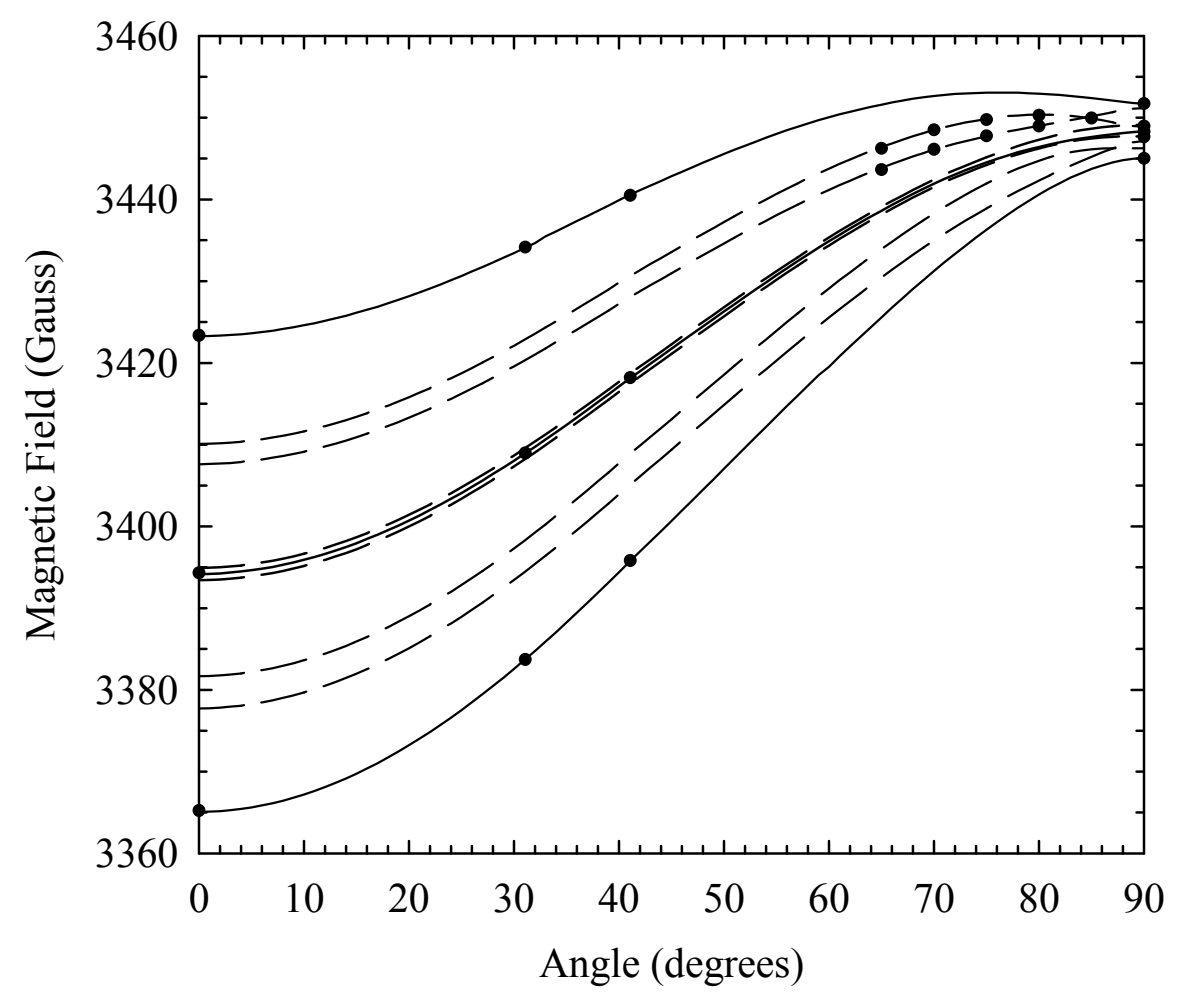

Figure 6.2. Angular dependence of the neutral nitrogen acceptor. Solid circles represent experimental data points. The solid lines and dashed lines represent the computer generated line positions of the "allowed transitions" (solid lines) and "forbidden transitions" (dashed lines) using the "best fit" parameters.

computer generated curves for the "allowed" and "forbidden" transitions using these "best-fit" values of the Hamiltonian parameters. Only the relative signs of the hyperfine and nuclear quadrupole parameters could be experimentally determined. A positive sign has been assigned to $\mathrm{A}$ because the nuclear $\mathrm{g}$ factor for the ${ }^{14} \mathrm{~N}$ nucleus is positive.

\subsubsection{Zinc Hyperfine}

Figure 6.3 shows the hyperfine interactions with the ${ }^{67} \mathrm{Zn}$ nuclei $(\mathrm{I}=5 / 2,4.1 \%$ abundant) surrounding the isolated nitrogen acceptor. These are the same data shown in Fig. 6.1, except the vertical scale is now significantly expanded to show the weaker ${ }^{67} \mathrm{Zn}$ 


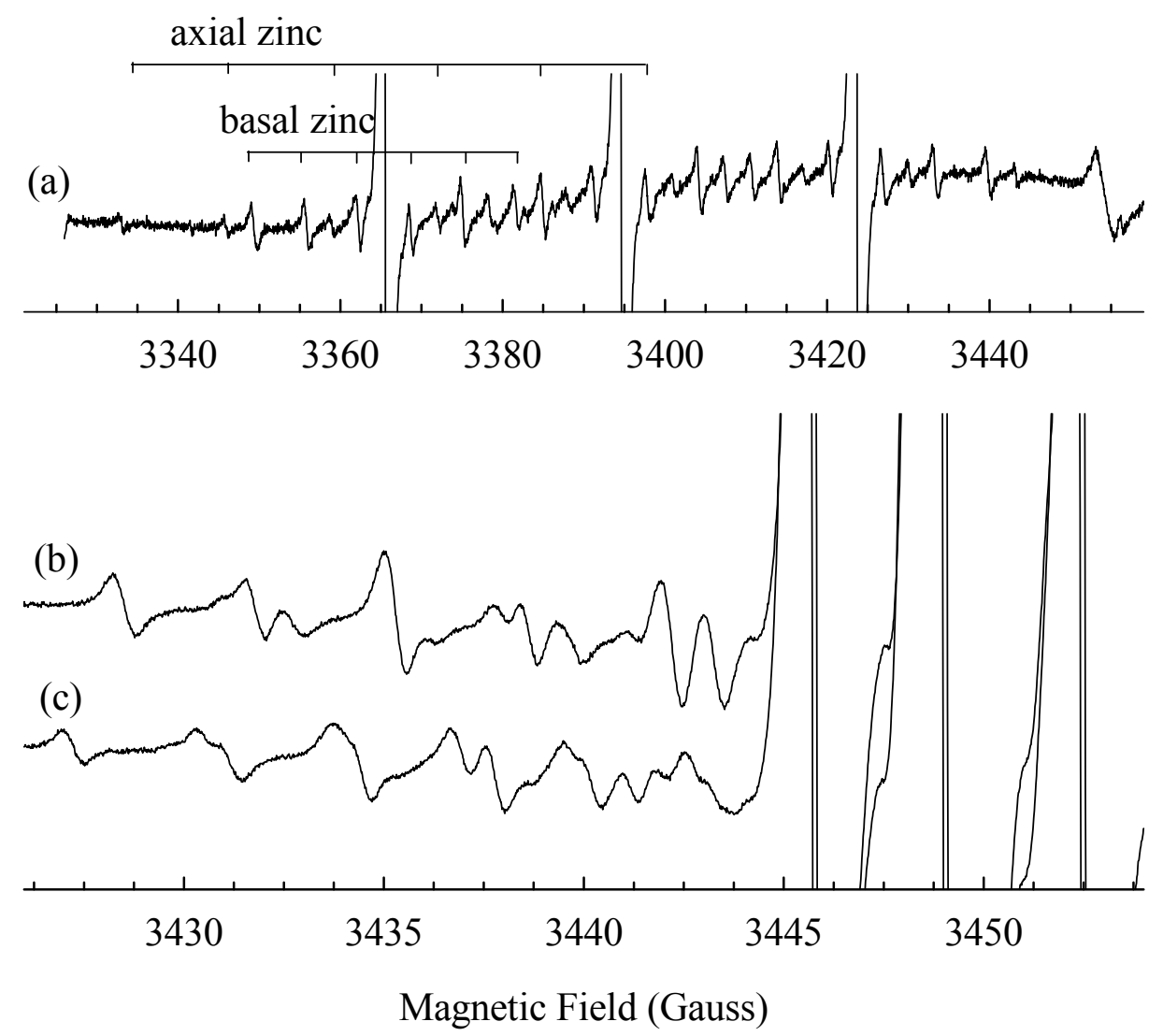

Figure 6.3. EPR spectra of the zinc hyperfine. The spectra were taken with the magnetic field (a) parallel to the $c$ axis, (b) perpendicular to the $c$ axis along "hs 1 ", and (c) perpendicular to the $c$ axis along "hs2".

hyperfine lines. For the magnetic field along the $c$ axis (Fig. 6.3(a)), the zinc hyperfine is easily resolved. There are two separate six-line spectra due to the ${ }^{67} \mathrm{Zn}$ for each of the three nitrogen hyperfine lines (a total of $36{ }^{67} \mathrm{Zn}$ lines). One six-line spectrum for each nitrogen line is due to a ${ }^{67} \mathrm{Zn}$ nucleus located at the axial zinc site above the nitrogen (labeled axial zinc). The hyperfine matrix for this spectrum has axial symmetry. The other six-line spectrum for each nitrogen line is due to a ${ }^{67} \mathrm{Zn}$ nucleus located at one of the three basal plane zinc sites (labeled nonaxial zinc). The basal plane neighbors have 
six crystallographically equivalent, but magnetically inequivalent, lattice sites. As the magnetic field is rotated away from the $c$ axis, the axial zinc spectrum shifts but does not split. The nonaxial zinc spectrum splits due to the different lattice sites, and give a different EPR spectrum for each of the two high symmetry directions that lie in the basal plane. The low field EPR lines of these two basal-plane spectra are shown in Figs. 6.3(b)

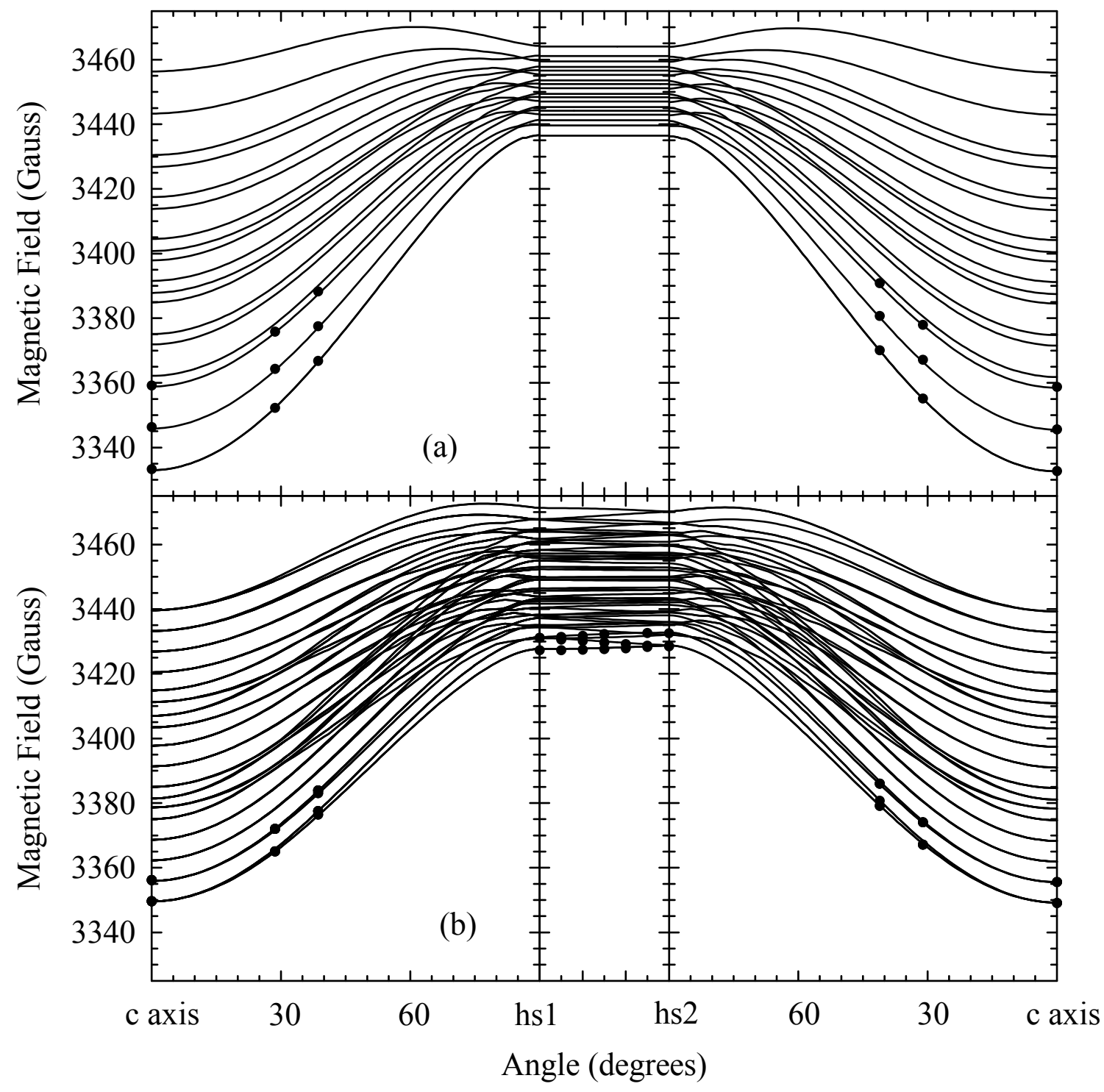

Figure 6.4. Angular dependence of (a) the axial zinc hyperfine and (b) the non axial zinc hyperfine. The data points represent experimental data, while the solid lines are computer generated curves using the "best fit" parameters. 
and 6.3(c). The axial zinc signals are not labeled in Figs. 6.3(b) and 6.3(c) because the hyperfine splitting is too small and the axial EPR lines cannot be separately identified.

Figure 6.4(a) shows the angular dependence of the axial ${ }^{67} \mathrm{Zn}$ hyperfine and Fig. 6.4(b) shows the dependence of the nonaxial ${ }^{67} \mathrm{Zn}$ hyperfine. The dots represent the experimental data. The following spin Hamiltonian was used to fit the data.

$$
\begin{aligned}
H=\beta \mathbf{B} \cdot \overrightarrow{\mathbf{g}} \cdot \hat{\mathbf{S}}+\hat{\mathbf{I}}^{\mathrm{N}} \cdot \overrightarrow{\mathbf{A}}^{\mathrm{N}} \cdot \hat{\mathbf{S}}+\hat{\mathbf{I}}^{\mathrm{Zn}} \cdot \overrightarrow{\mathbf{A}}^{\mathrm{Zn}} \cdot \hat{\mathbf{S}} \\
\quad \cdots+\hat{\mathbf{I}}^{\mathrm{N}} \cdot \overrightarrow{\mathbf{Q}}^{\mathrm{N}} \cdot \hat{\mathbf{I}}^{\mathrm{N}}-\mathrm{g}_{\mathrm{N}}^{\mathrm{N}} \beta_{\mathrm{N}} \mathbf{B} \cdot \hat{\mathbf{I}}^{\mathrm{N}}-\mathrm{g}_{\mathrm{N}}^{\mathrm{Zn}} \beta_{\mathrm{N}} \mathbf{B} \cdot \mathbf{I}^{\mathrm{Zn}}
\end{aligned}
$$

During the fitting procedure, the g values and the hyperfine and quadrupole values for nitrogen were fixed at the values determined earlier in this chapter. For the axial ${ }^{67} \mathrm{Zn}$, fitting to the data in Fig. 6.4 gave an axial hyperfine matrix with values of $A_{\|}=36.2$ $\mathrm{MHz}$ and $\mathrm{A}_{\perp}=11.3 \mathrm{MHz}$. For the non axial ${ }^{67} \mathrm{Zn}$, the resulting hyperfine values were $\mathrm{A}_{\mathrm{x}}$ $=14.8 \mathrm{MHz}, \mathrm{A}_{\mathrm{y}}=17.8 \mathrm{MHz}$, and $\mathrm{A}_{\mathrm{z}}=20.3 \mathrm{MHz}$ with Euler angles of $\theta=90^{\circ}, \varphi=-90^{\circ}$, $\psi=0^{\circ}$. The solid curves in Fig. 6.4 represent the lines generated from these "best fit" parameters. 


\section{References}

1. M. Asif Khan, M. Shatalov, H. P. Maruska, H. M. Wang, and E. Kuokstis, Japanese Journal of Applied Physics 44, 7191 (2005).

2. M. Razeghi, A. Yasan, R. McClintock, K. Mayes, D. Shiell, S. R. Darvish, and P. Kung, Physica Status Solidi (c) 1 , S141 (2004).

3. M. W. Bayerl, M. S. Brandt, T. Graf, O. Ambacher, J. A. Majewski, M.

Stutzmann, D. J. As, and K. Lischka, Physical Review B 모, 165204 (2001).

4. T. Mattila and R. M. Nieminen, Physical Review B 프, 16676 (1996).

5. C. G. Van de Walle, Physical Review B 57, R2033 (1998).

6. G. A. Slack, L. J. Schowalter, D. Morelli, and J. A. Freitas Jr., Journal of Crystal Growth $\underline{246}, 287$ (2002).

7. J. A. Freitas Jr, G. C. B. Braga, E. Silveira, J. G. Tischler, and M. Fatemi, Applied Physics Letters $\underline{83}, 2584$ (2003).

8. M. Bickermann, B. M. Epelbaum, and A. Winnacker, Physica Status Solidi (c) $\underline{0}$, $1993(2003)$.

9. Q. Zhou, M. O. Manasreh, M. Pophristic, S. Guo, and I. T. Ferguson, Applied Physics Letters $\underline{79}, 2901$ (2001).

10. Q. Zhou and M. O. Manasreh, Applied Physics Letters $\underline{\text { 80, }} 2072$ (2002).

11. J. M. Vail, D. K. Chevrier, R. Pandey, and M. A. Blanco, Journal of Physics: Condensed Matter 18, 2125 (2006).

12. C. Stampfl and C. G. Van de Walle, Physical Review B $\underline{65}, 155212$ (2002).

13. D. C. Look, Materials Science and Engineering B $\underline{80}, 383$ (2001).

14. S. J. Pearton, D. P. Norton, K. Ip, Y. W. Heo, and T. Steiner, Superlattices and Microstructures $\underline{34}, 3$ (2003).

15. S. J. Pearton, D. P. Norton, K. Ip, Y. W. Heo, and T. Steiner, Journal of Vacuum Science and Technology B 22, 932 (2004). 
16. S. J. Pearton, C. R. Abernathy, M. E. Overberg, G. T. Thaler, D. P. Norton, N. Theodoropoulou, A. F. Hebard, Y. D. Park, F. Ren, J. Kim, and L. A. Boatner, Journal of Applied Physics $\underline{\text { 93, }} 1$ (2003).

17. S. J. Pearton, W. H. Heo, M. Ivill, D. P. Norton, and T. Steiner, Semiconductor Science and Technology $\underline{19}$, R59 (2004).

18. D. C. Look, B. Claflin, Ya. I. Alivov, and S. J. Park, Physica Status Solidi (a) 201, 2203 (2004).

19. D. C. Look and B. Claflin, Physica Status Solidi (c) 1 , 624 (2004).

20. B. K. Meyer, H. Alves, D. M. Hofmann, W. Kriegseis, D. Forster, F. Bertram, J. Christen, A. Hoffmann, M. Strassburg, M. Dworzak, U. Haboeck, and A. V. Rodina, Physica Status Solidi (b) 241, 231 (2004).

21. O. F. Schirmer, Journal of Physics and Chemistry of Solids $\underline{29}, 1407$ (1968).

22. D. Galland and A. Herve, Physics Letters A $\underline{33}$, 1 (1970).

23. R. Dingle, Physical Review Letters $\underline{23}, 579$ (1969).

24. L. Wang and N. C. Giles, Applied Physics Letters $\underline{84}, 3049$ (2004).

25. J. M. Smith and W. E. Vehse, Physics Letters A $\underline{31,} 147$ (1970).

26. C. Gonzalez, D. Galland, and A. Herve, Physica Status Solidi (b) $\underline{72}, 309$ (1975).

27. V. Soriano and D. Galland, Physica Status Solidi (b) $\underline{77}, 739$ (1976).

28. K. Vanheusden, C. H. Seager, W. L. Warren, D. R. Tallant, and J. A. Voigt, Applied Physics Letters 68, 403 (1996).

29. K. Vanheusden, W. L. Warren, C. H. Seager, D. R. Tallant, J. A. Voigt, and B. E. Gnade, Journal of Applied Physics 79, 7983 (1996).

30. J. Schneider and A. Rauber, Zeitschrift fuer Naturforschung A $\underline{16}, 712$ (1961).

31. M. Schulz, Physica Status Solidi (a) 27, K5 (1975).

32. D. Block, A. Herve, and R. T. Cox, Physical Review B 25, 6049 (1982).

33. C. Gonzalez, D. Block, R. T. Cox, and A. Herve, Journal of Crystal Growth $\underline{59}$, 357 (1982). 
34. A. L. Taylor, G. Filipovich, and G. K. Lindeberg, Solid State Communications $\underline{8}$, 1359 (1970).

35. F. Leiter, H. Zhou, F. Henecker, A. Hofstaetter, D. M. Hofmann, and B. K. Meyer, Physica B $\underline{308-310}, 908$ (2001).

36. W. E. Carlos, E. R. Glaser, and D. C. Look, Physica B 308-310, 976 (2001).

37. G. N. Aliev, S. J. Bingham, D. Wolverson, J. J. Davies, H. Makino, H. J. Ko, and T. Yao, Physical Review B $\underline{70}, 115206$ (2004).

38. Ya. I. Alivov, M. V. Chukichev, and V. A. Nikitenko, Semiconductors $\underline{38}, 31$ (2004).

39. H. S. Kang, J. S. Kang, J. W. Kim, and S. Y. Lee, Journal of Applied Physics $\underline{95}$, 1246 (2004).

40. M. R. Phillips, O. Gelhausen, and E. M. Goldys, Physica Status Solidi (a) 201, 229 (2004).

41. Q. P. Wang, D. H. Zhang, Z. Y. Xue, and X. J. Zhang, Optical Materials 26 23 (2004).

42. D. Li, Y. H. Leung, A. B. Djurisic, Z. T. Liu, M. H. Xie, S. L. Shi, S. J. Xu, and W. K. Chan, Applied Physics Letters $\underline{85}, 1601$ (2004).

43. N. Y. Garces, L. Wang, L. Bai, N. C. Giles, L. E. Halliburton, and G. Cantwell, Applied Physics Letters $\underline{81}, 622$ (2002).

44. R. E. Dietz, H. Kamimura, M. D. Sturge, and A. Yariv, Physical Review 132, 1559 (1963).

45. D. C. Reynolds, D. C. Look, and B. Yogai, Journal of Applied Physics $\underline{\text { 89, }} 6189$ (2001).

46. J. A. Weil, J. R. Bolton, and J. E. Wertz, Electron Paramagnetic Resonance: Elementary Theory and Practical Applications (John Wiley \& Sons, New York, 1994). 
47. J. -M. Spaeth, J. R. Niklas, R. H. Bartram, Structural Analysis of Point Defects in Solids (Springer-Verlag, Berlin, 1992).

48. S. D. Setzler, Ph.D. Dissertation, West Virginia University (1998).

49. J. E. Wertz and J. R. Bolton, Electron Spin Resonance: Elementary Theory and Applications (McGraw-Hill, New York, 1972).

50. M. Honda, K. Atobe, N. Fukuoka, M. Okada, and M. Nakagawa, Japanese Journal of Applied Physics, Part 2 29, L652 (1990).

51. S. Schweizer, U. Rogulis, J.-M. Spaeth, L. Trinkler, and B. Berzina, Physica Status Solidi B 219, 171 (2000).

52. U. Ozgur, Y. I. Alivov, C. Liu, A. Teke, M. A. Reshchikov, S. Dogan, V. Avrutin, S. J. Cho, and H. Morkoc, Journal of Applied Physics 98, 041301 (2005).

53. G. A. Jeffery and G. S. Parry, Journal of Chemical Physics 23, 406 (1955).

54. G. A. Slack and T. F. McNelly, Journal of Crystal Growth $\underline{34}, 263$ (1976); ibid. $\underline{42}, 560$ (1977).

55. J. C. Rojo, G. A. Slack, K. Morgan, B. Raghothamachar, M. Dudley, and L. J. Schowalter, Journal of Crystal Growth $\underline{231}, 317$ (2001).

56. R. Schlesser and Z. Sitar, Journal of Crystal Growth 234, 349 (2002).

57. J. C. Rojo, L. J. Schowalter, R. Gaska, M. Shur, M. A. Khan, J. Yang, and D. D. Koleske, Journal of Crystal Growth 240, 508 (2002).

58. M. Strassburg, J. Senawiratne, N. Dietz, U. Haboeck, A. Hoffmann, V. Noveski, R. Dalmau, R. Schlesser, and Z. Sitar, Journal of Applied Physics 96, 5870 (2004).

59. C. G. Van de Walle and J. Neugebauer, Journal of Applied Physics $\underline{95}, 3851$ (2004).

60. K. Atobe, M. Honda, N. Fukuoka, M. Okada, and M. Nakagawa, Japanese Journal of Applied Physics, Part $1 \underline{29}, 150$ (1990). 
61. P. M. Mason, H. Przybylinska, G. D. Watkins, W. J. Choyke, and G. A. Slack, Physical Review B 59, 1937 (1999).

62. J. Burm, W. J. Chaff, L. F. Eastman, H. Amano, and I. Akasaki, Applied Physics Letters $\underline{68}, 2849$ (1996).

63. Deep Centers in Semiconductors, edited by Sokrates T. Pantelides, (Gordon and Breach, Yverdon, 1992).

64. D. V. Lang, R. A. Logan, and M. Jaros, Physical Review B 19, 1015 (1979).

65. D. V. Lang and R. A. Logan, Physical Review Letters $\underline{\text { 39, }} 635$ (1977).

66. R. J. Nelson, Applied Physics Letters 31, 351 (1977).

67. V. Narayanamurti, R. A. Logan, and M. A. Chin, Physical Review Letters $\underline{43}$, 1536 (1979).

68. B. Ballard, G. Vincent, D. Bois, and P. Hirtz, Applied Physics Letters $\underline{34}, 108$ (1979).

69. R. A. Craven and D. Finn, Journal of Applied Physics 50, 6334 (1979).

70. M. G. Craford, G. E. Stillman, J. A. Rossi, and N. Holonyak, Jr., Physical Review $\underline{168}, 867$ (1968).

71. R. Zeisel, M. W. Bayerl, S. T. B. Goennenwein, R. Dimitrov, O. Ambacher, M. S. Brandt, and M. Stutzmann, Physical Review B $\underline{61}$, R16283 (2000)

72. M. S. Brandt, R. Zeisel, S. T. B. Gönnenwein, M. W. Bayerl, and M. Stutzmann, Physica Status Solidi (b) 235, 13 (2002).

73. C. H. Park and D. J. Chadi, Physical Review B 55, 12995 (1997).

74. P. Bogulawski and J. Bernholc, Physical Review B ㅌ6, 9496 (1997).

75. C. Skierbiszewski, T. Suski, M. Leszczyznski, M. Shin, M. Sokoronski, M. D. Bremser, and R.F. Davis, Applied Physics Letters 74, 3833 (1999).

76. C. Wetzel, T. Suski, J. W. Ager III, E. R. Weber, E. E. Haller, S. Fischer, B. K. Meyer, R. J. Molnar, and P. Perlin, Physical Review Letters $\underline{78}, 3923$ (1997).

77. H. Karzel et al., Physical Review B 포, 11425 (1996). 
78. Y. Jiang, N. C. Giles, and L. E. Halliburton, Journal of Applied Physics 101, 093706 (2007); K. Maeda, M. Sato, I. Niikura, and T. Fukuda, Semiconductor Science and Technology 20, S49 (2005).

79. L. E. Halliburton, L. Wang, L. Bai, N. Y. Garces, N. C. Giles, M. J. Callahan, and B. Wang, Journal of Applied Physics $\underline{96}, 7168$ (2004).

80. J. Nause, III-Vs Review 12, 28 (1999).

81. L. E. Halliburton, N. C. Giles, N. Y. Garces, M. Luo, C. Xu, L. Bai, and L. A. Boatner, Applied Physics Letters $\underline{87}, 172108$ (2005).

82. S. Lany and A. Zunger, Physical Review B 72, 035215 (2005).

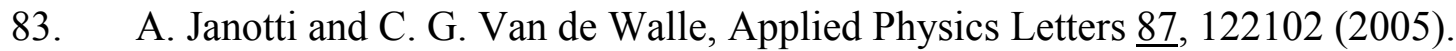

84. F. Tuomisto, K. Saarinen, D. C. Look, and G. C. Farlow, Physical Review B $\underline{72}$, $085206(2005)$.

85. K. Fink, Physical Chemistry Chemical Physics. ㄱ, 2999 (2005).

86. S. Lany and A. Zunger, Physical Review Letters $\underline{98}, 045501$ (2007).

87. A. Janotti and C. G. Van de Walle, Nature Materials $\underline{6}$, 44 (2007).

88. H. Takenaka and D. J. Singh, Physical Review B $\underline{75}$, 241102 (2007).

89. F. A. Selim, M. H. Weber, D. Solodovnikov, and K. G. Lynn, Physical Review Letters $\underline{99}, 085502$ (2007).

90. J. M. Meese and D. R. Locker, Solid State Communications 11, 1547 (1972).

91. D. R. Locker and J. M. Meese, IEEE Transactions on Nuclear Science 19, 237 (1972).

92. K. Leutwein and J. Schneider, Zeitschrift Naturforschung 26a, 1236 (1971).

93. B. Schallenberger and A. Hausmann, Zeitschrift Physik B 23, 177 (1976).

94. D. Galland and A. Herve, Solid State Communications 14, 953 (1974).

95. Yu. V. Gorelkinskii and G. D. Watkins, Physical Review B 9ㅜ, 115212 (2004).

96. L. S. Vlasenko and G. D. Watkins, Physical Review B $\underline{71}, 125210$ (2005).

97. L. S. Vlasenko and G. D. Watkins, Physical Review B $\underline{72,} 035203$ (2005). 


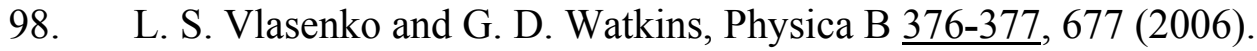

99. D. C. Look, D. C. Reynolds, J. R. Sizelove, R. L. Jones, C. W. Litton, G. Cantwell, and W. C. Harsch, Solid State Communications 105, 399 (1998).

100. L. A. Kappers, O. R. Gilliam, S. M. Evans, L. E. Halliburton, and N. C. Giles (to be published in Nuclear Instruments and Methods, 2008).

101. G. Heiland, E. Mollwo, and F. Stockmann, in Solid State Physics, edited by F. Seitz and D. Turnbull (Academic, New York, 1959), Vol. 8, p. 205.

102. W. E. Vehse, W. A. Sibley, F. J. Keller, and Y. Chen, Physical Review 167, 828 (1968).

103. R. M. de la Cruz, R. Pareja, R. Gonzalez, L. A. Boatner, and Y. Chen, Physical Review B $\underline{45}, 6581$ (1992).

104. J. R. Harbridge, G. A. Rinard, R. W. Quine, S. S. Eaton, and G. R. Eaton, Journal of Magnetic Resonance 156, 41 (2002).

105. W. W. Walsh and L. W. Rupp, Physical Review 126, 952 (1962).

106. M. Birkholz, P. Kanschat, T. Weiss, M. Czerwensky, and K. Lips, Physical Review B 59, 12268 (1999).

107. W. Gehlhoff, D. Azamat, A. Hoffmann, N. Dietz, and O. V. Voevodina, Physica B $\underline{376-377}, 790$ (2006).

108. L. E. Halliburton, B. D. Perlson, R. A. Weeks, J. A. Weil, and M. C. Wintersgill, Solid State Communications $\underline{30}, 575$ (1979).

109. P. W. Kirklin, P. Auzins, and J. E. Wertz, Journal of Physics and Chemistry of Solids $\underline{26}, 1067$ (1965).

110. G. A. Shi, M. Saboktakin, M. Stavola, and S. J. Pearton, Applied Physics Letters $\underline{85}, 5601$ (2004).

111. J. Schneider, A. Rauber, B. Dischler, T. L. Estle, and W. C. Holton, Journal of Chemical Physics $\underline{42}, 1839$ (1965).

112. J. Bittebierre and R. T. Cox, Physical Review B 34 2360 (1986). 
113. J. Gebauer, R. Krause-Rehberg, M. Prokesch, and K. Irmscher, Physical Review B $\underline{66}, 115206$ (2002).

114. W. P. Unruh, Y. Chen, and M. M. Abraham, Physical Review Letters $\underline{30}, 446$ (1973).

115. R. C. DuVarney and A. K. Garrison, Solid State Communications 12, 1235 (1973).

116. M. M. Abraham, Y. Chen, and W. P. Unruh, Physical Review B $\underline{9}, 1842$ (1974).

117. V. A. Nikitenko, K. E. Tarkpea, S. F. Nikulshin, and I. P. Kuzmina, Zh. Prikl. Spektrosk. $\underline{47}, 834$ (1987).

118. V. A. Nikitenko, Zh. Prikl. Spektrosk. 57, 367 (1992).

119. J. M. Smith, Ph.D. Dissertation, West Virginia University (1969).

120. W. E. Carlos, E. R. Glaser, and D. C. Look, Physica B 308-310, 976 (2001).

121. N. Y. Garces, N. C. Giles, L. E. Halliburton, G. Cantwell, D. B. Eason, D. C. Reynolds, and D. C. Look, Applied Physics Letters 무, 1334 (2002).

122. N. Y. Garces, L. Wang, N. C. Giles, L. E. Halliburton, G. Cantwell, and D. B. Eason, Journal of Applied Physics 94, 519 (2003). 